\title{
Isomorphism of Intransitive Linear Lie Equations ${ }^{\star}$
}

\author{
Jose Miguel Martins VELOSO
}

Faculdade de Matematica, UFPA, Belem, PA, CEP 66075-110, Brasil

E-mail:veloso@ufpa.br

Received February 09, 2009, in final form November 11, 2009; Published online November 17, 2009 doi:10.3842/SIGMA.2009.103

\begin{abstract}
We show that formal isomorphism of intransitive linear Lie equations along transversal to the orbits can be extended to neighborhoods of these transversal. In analytic cases, the word formal is dropped from theorems. Also, we associate an intransitive Lie algebra with each intransitive linear Lie equation, and from the intransitive Lie algebra we recover the linear Lie equation, unless of formal isomorphism. The intransitive Lie algebra gives the structure functions introduced by É. Cartan.
\end{abstract}

Key words: Lie equations; Lie groupoids; intransitive; isomorphism

2000 Mathematics Subject Classification: 58H05; 58H10

\section{Introduction}

It is known [7] that the isomorphism of the fibers of transitive linear Lie equations at two points is sufficient to obtain the formal isomorphism of the Lie equations. This is proved by constructing a system of partial differential equations (SPDE) whose solutions would be these isomorphisms. This SPDE may be not integrable [2,11], although it is formally integrable. If the data are analytic, the SPDE is integrable.

In this paper we consider principally the extension of this theorem to intransitive linear Lie equations. Intransitive linear Lie equations generate a family of orbits on the manifold that we suppose locally to be a foliation. Given two intransitive Lie equations, consider the restriction of both Lie equations along two transversal to the orbits. If these restrictions are isomorphic in a certain sense, then we can construct a formally integrable SPDE whose solutions (if they exist) are isomorphisms of the two Lie equations. Therefore we prove, at least in the analytic case, that formal isomorphism of two linear Lie equations along transversal to the orbits can be extended locally to local isomorphism of the two linear Lie equations in a neighborhood of the two transversal.

Specifically, consider $M$ and $M^{\prime}$ manifolds of the same dimension, $V$ and $V^{\prime}$ integrable distributions of the same dimension on $M$ and $M^{\prime}$, respectively, $N$ and $N^{\prime}$ submanifolds of $M$ and $M^{\prime}$ such that each integral leaf of $V$ and $V^{\prime}$ through points $x \in M$ and $x^{\prime} \in M^{\prime}$ intersect $N$ and $N^{\prime}$ (at least locally) at unique points $\rho x$ and $\rho^{\prime} x^{\prime}$, respectively. Let be $\phi: N \rightarrow N^{\prime}$ a local diffeomorphism, $a \in N, a^{\prime} \in N^{\prime}, \phi(a)=a^{\prime}, Q_{\phi}^{k}$ the manifold of $k$-jets of local diffeomorphisms $f: M \rightarrow M^{\prime}$ such that $\phi \rho(x)=\rho^{\prime} f(x)$, and $\mathcal{Q}_{\phi}^{k}$ the sheaf of germs of invertible local sections of $Q_{\phi}^{k}$. Furthermore, let be $R^{k} \subset J^{k} V, R^{\prime k} \subset J^{k} V^{\prime}$ intransitive linear Lie equations such that $R^{0}=J^{0} V, R^{\prime 0}=J^{0} V^{\prime}$. We say that $R^{k}$ at point $a$ is formally isomorphic to $R^{\prime k}$ at a point $a^{\prime}$ if we can construct a formally integrable SPDE $S^{k} \subset Q_{\phi}^{k}$ such that any solution $f$ of $S^{k}$ satisfies $\left(j^{k+1} f\right)_{*}\left(R^{k}\right)=R^{\prime k}$ (see Definition 5.3). We prove in Proposition 5.3 that this condition is

\footnotetext{
*This paper is a contribution to the Special Issue "Élie Cartan and Differential Geometry". The full collection is available at http://www.emis.de/journals/SIGMA/Cartan.html
} 
equivalent to the existence of $F \in \mathcal{Q}_{\phi}^{k+1}$ such that $\beta F(a)=a^{\prime}$ and $F_{*}\left(T M \oplus R^{k}\right)=T M^{\prime} \oplus R^{\prime k}$. Then we prove:

Theorem 5.1. Let be $\Phi: N \rightarrow Q_{\phi}^{k+1}$ such that $\beta \Phi=\phi$ and

$$
\Phi_{*}\left(\left.T N \oplus R^{k}\right|_{N}\right)=\left.T N^{\prime} \oplus R^{\prime k}\right|_{N^{\prime}}
$$

Then given a diffeomorphism $f: M \rightarrow M^{\prime}$ such that $f_{*} V=V^{\prime}$ and $\left.f\right|_{N}=\phi$ there exists $F \in \mathcal{Q}_{\phi}^{k+1}$ satisfying $\left.F\right|_{N}=\Phi, \beta F=f$ and

$$
F_{*}\left(T M \oplus R^{k}\right)=T M^{\prime} \oplus R^{\prime k} .
$$

This theorem for transitive linear Lie equations is in [7].

The next step is to define an intransitive Lie algebra representing $\left.T N \oplus R^{k}\right|_{N}$. Transitive Lie algebras were defined in [10] as the algebraic object necessary to study transitive infinitesimal Lie pseudogroups. This program was pursued in the papers $[6,7,8,9,14,16,17,20,24]$ disclosing the fitness of transitive Lie algebras to study transitive linear Lie equations.

At the same time, several tries were made to include intransitive linear Lie equations in this theory $[12,13,18,19]$. Basically, they associated a family of transitive Lie algebras on a transversal to the orbits with an intransitive infinitesimal Lie pseudogroup, each transitive Lie algebra corresponding to the transitive infinitesimal Lie pseudogroup obtained by restriction to each orbit of the infinitesimal intransitive Lie pseudogroup. Another approach was to study the Lie algebra of infinite jets at one point of the infinitesimal intransitive Lie pseudogroup; this algebra is bigraded, and this bigraduation may give the power series of the structure functions introduced in [1]. In the first approach, it may happen to be impossible to relate transitive Lie algebras along the transversal and give a continuous structure to this family, as the following example in [27] shows: let be $\Theta$ the infinitesimal intransitive Lie pseudogroup acting in the plane $\mathbb{R}^{2}$ given by

$$
\Theta=\left\{\theta(x, y) \frac{\partial}{\partial y} \text { such that } \frac{\partial \theta}{\partial y}=a(x) \frac{\partial \theta}{\partial x} \text { with } a \neq 0, a(0)=0\right\} .
$$

Two of these infinitesimal intransitive Lie pseudogroups given by functions $a(x)$ and $\bar{a}(x)$ are isomorphic if and only if there exists a $C^{\infty}$-function $b(x)$, with $b(0) \neq 0$ such that $\bar{a}(x)=b(x) a(x)$. The restriction of $\Theta$ to the orbits $\left\{\left(x_{0}, y\right): y \in \mathbb{R}\right\}$, with $a\left(x_{0}\right) \neq 0$ is the infinitesimal Lie pseudogroup of differentiable vector fields on $\mathbb{R}$, and the restriction to the orbit where $a\left(x_{0}\right)=0$ are the infinitesimal translations on $\mathbb{R}$. This example shows that, even if the intransitive linear Lie equation associated to $\Theta$ has all properties of regularity, by choosing an appropriate function $a(x)$, we obtain a highly discontinuous family of transitive Lie algebras along the transversal. If $a(x)$ and $\bar{a}(x)$ have the same power expansion series around 0, the bigraded Lie algebras of infinite jets of vector fields of $\Theta$ and $\bar{\Theta}$ at point $(0,0)$ are the same. So, the bigraded Lie algebras cannot distinguish between non isomorphic intransitive Lie pseudogroups. However, É. Cartan associated "structure functions" constant along the orbits with infinitesimal Lie pseudogroups. Therefore any definition of intransitive Lie algebras must contain a way of getting the germs of "structure functions" at the point considered.

A way to define such algebra is the following: let be $\mathcal{O}_{N, a}$ the ring of germs of real functions defined on $N$ at the point $a, L_{m}$ the $\mathcal{O}_{N, a}$-module of germs of sections of $\left.R^{m}\right|_{N}$ at point $a$, and $\mathcal{T} N_{a}$ the $\mathcal{O}_{N, a}$-module of germs of sections of $T N$ at point $a$. The vector bundles $R^{m}$, with $m>k$, are the prolongations of $R^{k}$, which we suppose formally integrable. There is a $\mathbb{R}$-bilinear map

$$
\llbracket, \rrbracket_{m}:\left(\mathcal{T} N_{a} \oplus L_{m}\right) \times\left(\mathcal{T} N_{a} \oplus L_{m}\right) \rightarrow\left(\mathcal{T} N_{a} \oplus L_{m-1}\right),
$$


given by: the bracket of germs of vector fields in $\mathcal{T} N_{a}$; the germ of $i(v) D \xi$ for $v \in \mathcal{T} N_{a}, \xi \in L_{m}$ and $D$ the linear Spencer operator; and the $\mathcal{O}_{N, a}$-bilinear map defined on holonomic sections by $\llbracket j^{k} \theta, j^{k} \eta \rrbracket_{k}=j^{k-1}[\theta, \eta]$. Let be $L=\operatorname{limproj}_{m} L_{m}, \mathcal{L}=\mathcal{T} N_{a} \oplus L$ and $\llbracket, \rrbracket_{\infty}=\operatorname{limproj}_{m} \llbracket, \rrbracket_{m}$. Then $\left(\mathcal{L}, \mathbb{\llbracket}, \rrbracket_{\infty}\right)$ is the $\mathcal{O}_{N, a}$-intransitive $\mathbb{R}$-Lie algebra associated with the formally integrable linear Lie equation $R^{k}$ at the point $a$. From $\left(\mathcal{L}, \llbracket, \rrbracket_{\infty}\right)$ we can obtain the germ of $\left.R^{k}\right|_{N}$ at point $a$, and, by applying the Theorem of [25] and Theorem 5.1 of this paper, we get the germ of the linear Lie equation $R^{k}$ at the point $a$, up to a formal isomorphism (cf. Theorems 6.1 and 6.2 below).

We summarize the content of this paper. Section 2 presents basic facts on groupoids and algebroids of jets, the calculus on the diagonal introduced in [16, 17], and the construction of the first linear and non-linear Spencer complexes. We tried to be as complete as possible, and the presentation emphasizes the geometric relationship between the first linear and non-linear Spencer operators, and the left and right actions of a groupoid on itself. We hope this section will facilitate the reading of this paper, since several formulas given here are sometimes not easily identified in $[16,17,14,6,7]$, due to the simultaneous use of the first, second and the sophisticated non-linear Spencer complex, and the identifications needed to introduce them.

Section 3 contains the construction of partial connections on $J^{k} V$. These partial connections are fundamental for Section 5. Section 4 introduces the basic facts on linear Lie equations and the associated groupoids. Section 5 presents the definition of formal isomorphism of linear Lie equations, and the proof of Theorem 5.1. Section 6 introduces the definition of intransitive Lie algebras, and the notion of isomorphism of these algebras. The last section discuss the classification of intransitive linear Lie equations of order one in the plane, with symbol $g^{1}$ of dimension one. This classification contains the examples introduced above.

\section{Preliminaries}

In this section, we present some background material. The main references for this section are $[16,17,14]$, and we will try to maintain the exposition as self-contained as possible, principally introducing geometrical proofs for actions of invertible sections of $Q^{k+1} M$ on sections of $T \oplus J^{k} T M$.

\subsection{Groupoids and algebroids of jets}

Let be $M$ a manifold and $Q^{k} M=Q^{k}$ the manifold of $k$-jets of local diffeomorphisms of $M$. This manifold has a natural structure of Lie groupoid given by composition of jets

$$
j_{f(x)}^{k} g \cdot j_{x}^{k} f=j_{x}^{k}(g f),
$$

and inversion

$$
\left(j_{x}^{k} f\right)^{-1}=j_{f(x)}^{k} f^{-1},
$$

where $f: U \rightarrow V, g: V \rightarrow W$ are local diffeomorphisms of $M$, and $x \in U$. The groupoid $Q^{k}$ has a natural submanifold of identities $I=j^{k} \mathrm{id}$, where id is the identity function of $M$. Then we have a natural identification of $M$ with $I$, given by $x \mapsto I(x)$. Therefore we can think of $M$ as a submanifold of $Q^{k}$. There are two submersions $\alpha, \beta: Q^{k} \rightarrow M$, the canonical projections source, $\alpha\left(j_{x}^{k} f\right)=x$, and target, $\beta\left(j_{x}^{k} f\right)=f(x)$. We also consider $\alpha$ and $\beta$ with values in $I$, by the above identification of $M$ with $I$.

There are natural projections $\pi_{l}^{k}=\pi_{l}: Q^{k} \rightarrow Q^{l}$, for $k \geq l \geq 0$, defined by $\pi_{l}\left(j_{x}^{k} f\right)=j_{x}^{l} f$. Observe that $Q^{0}=M \times M$ and $\pi_{0}^{k}=(\alpha, \beta)$. The projections $\pi_{l}^{k}$ commute with the operations of composition and inversion in $Q^{k}$. 
We denote by $Q^{k}(x)$ the $\alpha$-fiber of $Q^{k}$ on $x \in M$, or $Q^{k}(x)=\alpha^{-1}(x)$; by $Q^{k}(\cdot, y)$ the $\beta$-fiber of $Q^{k}$ on $y \in M$, in another way, $Q^{k}(\cdot, y)=\beta^{-1}(y)$ and $Q^{k}(x, y)=Q^{k}(x) \cap Q^{k}(\cdot, y)$. The set $Q^{k}(x, x)$ is a group, the so-called isotropy group of $Q^{k}$ at point $x$. If $U, V$ are open sets of $M$, $Q^{k}(U)=\cup_{x \in U} Q^{k}(x), Q^{k}(\cdot, V)=\cup_{y \in V} Q^{k}(\cdot, y)$, and $Q^{k}(U, V)=Q^{k}(U) \cap Q^{k}(\cdot, V)$.

A (differentiable) section $F$ of $Q^{k}$ defined on an open set $U$ of $M$ is a differentiable map $F: U \rightarrow Q^{k}$ such that $\alpha(F(x))=x$. If $\beta(F(U))=V$ and $f=\beta F: U \rightarrow V$ is a diffeomorphism, we say that the section $F$ is invertible. We write $U=\alpha(F)$ and $V=\beta(F)$. An invertible section $F$ of $Q^{k}$ is said holonomic if there exists a diffeomorphism $f: \alpha(F) \rightarrow \beta(F)$ such that $F=j^{k} f$. In this case, $\beta F=f$.

We denote by $\mathcal{Q}^{k}$ the set of invertible sections of $Q^{k}$. Naturally $\mathcal{Q}^{k}$ has a structure of groupoid. If $F, H \in \mathcal{Q}^{k}$ with $\beta(F)=\alpha(H)$, then $H F(x)=H(f(x)) F(x)$ and $F^{-1}(y)=F\left(f^{-1}(y)\right)^{-1}$, $y \in \beta(F)$.

Similarly, we can introduce the groupoid of $l$-jets of invertible sections of $Q^{k}$, and we denote this groupoid by $Q^{l} Q^{k}$. We have the inclusions

$$
\begin{aligned}
\lambda^{l}: & Q^{k+l} \rightarrow Q^{l} Q^{k}, \\
& j_{x}^{k+l} F \mapsto j_{x}^{l} j^{k} F .
\end{aligned}
$$

An invertible section $F$, with $\alpha(F)=U, \beta(F)=V$, defines a diffeomorphism

$$
\begin{gathered}
\tilde{F}: \quad Q^{k}(\cdot, U) \rightarrow Q^{k}(\cdot, V), \\
X \mapsto F(\beta(X)) X .
\end{gathered}
$$

The differential $\tilde{F}_{*}: T Q^{k}(\cdot, U) \rightarrow T Q^{k}(\cdot, V)$ depends, for each $X \in Q^{k}(\cdot, U)$, only on $j_{\beta(X)}^{1} F$. This defines an action

$$
\begin{aligned}
j_{\beta(X)}^{1} F: & T_{X} Q^{k} \rightarrow T_{F(\beta(X)) X} Q^{k}, \\
& v \mapsto j_{\beta(X)}^{1} F \cdot v=\left(\tilde{F}_{*}\right)_{X}(v) .
\end{aligned}
$$

Then (2.2) defines a left action of $Q^{1} Q^{k}$ on $T Q^{k}$

$$
\begin{aligned}
& Q^{1} Q^{k} \times T Q^{k} \rightarrow T Q^{k}, \\
& \left(j_{\beta(X)}^{1} F, v \in T_{X} Q^{k}\right) \mapsto j_{\beta(X)}^{1} F \cdot v \in T_{F(\beta(X)) X} Q^{k} .
\end{aligned}
$$

If $V_{\beta}^{k} \equiv \operatorname{ker} \beta_{*} \subset T Q^{k}$ denotes the subvector bundle of $\beta_{*}$ vertical vectors, then the action (2.2) depends only on $F(\beta(X))$ :

$$
\begin{aligned}
& Q^{k} \times V_{\beta}^{k} \rightarrow V_{\beta}^{k}, \\
& \left(F(\beta(X)), v \in\left(V_{\beta}^{k}\right)_{X}\right) \mapsto F(\beta(X)) \cdot v \in\left(V_{\beta}^{k}\right)_{F(\beta(X)) X} .
\end{aligned}
$$

In a similar way, $F$ defines a right action which is a diffeomorphism

$$
\begin{aligned}
\bar{F}: & Q^{k}(V) \rightarrow Q^{k}(U), \\
& X \mapsto X F\left(f^{-1}(\alpha(X))\right) .
\end{aligned}
$$

The differential $\bar{F}_{*}$ of $\bar{F}$ induces the right action

$$
\begin{aligned}
& T Q^{k} \times Q^{1} Q^{k} \rightarrow T Q^{k}, \\
& \left(v \in T_{X} Q^{k}, j_{f^{-1}(\alpha(X))}^{1} F\right) \mapsto v \cdot j_{f^{-1}(\alpha(X))}^{1} F=\left(\bar{F}_{*}\right)_{X}(v) .
\end{aligned}
$$


As $\beta(Y X)=\beta(Y)$, it follows that

$$
\beta_{*}\left(v \cdot j_{f^{-1}(\alpha(X))}^{1} F\right)=\beta_{*}(v),
$$

where $\beta_{*}: T Q^{k} \rightarrow T M$ is the differential of $\beta: Q^{k} \rightarrow M$. We verify from (2.5) that the function $\bar{F}$ restricted to the $\alpha$-fiber $Q^{k}(y)$ depends only on the value of $F$ in $f^{-1}(y)$. If $V_{\alpha}^{k}=$ ker $\alpha_{*}$, then the right action (2.6) depends only on the value of $F$ at each point, and the action (2.6) by restriction gives the action

$$
\begin{aligned}
& V_{\alpha}^{k} \times Q^{k} \rightarrow V_{\alpha}^{k}, \\
& \left(v \in\left(V_{\alpha}^{k}\right)_{X}, Y \in Q^{k}(\cdot, \alpha(X))\right) \mapsto v \cdot Y \in\left(V_{\alpha}^{k}\right)_{X Y} .
\end{aligned}
$$

A vector field $\bar{\xi}$ on $Q^{k}$ with values in $V_{\alpha}^{k}$ is said right invariant if $\bar{\xi}(X Y)=\bar{\xi}(X) \cdot Y$. The vector field $\bar{\xi}$ is determined by its restriction $\xi$ to $I$.

Let be $T=T M$ the tangent bundle of $M$, and $\mathcal{T}$ the sheaf of germs of local sections of $T$. We denote by $J^{k} T$ the vector bundle of $k$-jets of local sections of $\pi: T \rightarrow M$. Then $J^{k} T$ is a vector bundle on $M$, and we also denote by $\pi: J^{k} T \rightarrow M$ the map $\pi\left(j_{x}^{k} \theta\right)=x$. If $\theta: U \subset M \rightarrow T M$ is a local section, and $f_{t}$ is the 1-parameter group of local diffeomorphisms of $M$ such that $\left.\frac{d}{d t} f_{t}\right|_{t=0}=\theta$, then we get, for $x \in U$,

$$
\left.\frac{d}{d t} j_{x}^{k} f_{t}\right|_{t=0}=j_{x}^{k} \theta
$$

This means we have a natural identification

$$
\left.V_{\alpha}^{k}\right|_{I}=J^{k} T
$$

Therefore, as $\left.T Q^{k}\right|_{I}=\left.T I \oplus V_{\alpha}^{k}\right|_{I}$,

$$
\left.T Q^{k}\right|_{I} \cong T \oplus J^{k} T \text {, }
$$

and if we denote by

$$
\check{J}^{k} T=T \oplus J^{k} T,
$$

we have $\left.T Q^{k}\right|_{I} \cong \check{J}^{k} T$. Observe that $\check{J}^{k} T$ is a vector bundle on $M$. The restriction of $\beta_{*}$ : $T Q^{k} \rightarrow T$ to $\left.T Q^{k}\right|_{I}$, and the isomorphism $\left.T Q^{k}\right|_{I} \cong \check{J}^{k} T$ defines the map

$$
\begin{aligned}
\beta_{*}: & \check{J}^{k} T \rightarrow T, \\
& v+j_{x}^{k} \theta \in\left(\check{J}^{k} T\right)_{x} \mapsto v+\theta(x),
\end{aligned}
$$

which we denote again by $\beta_{*}$. For more details on this identification and the map $\beta_{*}$, see the Appendix of [14], in particular pages 260 and 274.

If $\xi$ is a section of $J^{k} T$ on $U \subset M$, let be

$$
\bar{\xi}(X)=\xi(\beta(X)) \cdot X
$$

the right invariant vector field on $Q^{k}(\cdot, U)$. Then $\bar{\xi}$ has $\bar{F}_{t},-\epsilon<t<\epsilon$, as the 1-parameter group of diffeomorphisms induced by invertible sections $F_{t}$ of $Q^{k}$ such that

$$
\left.\frac{d}{d t} \bar{F}_{t}\right|_{t=0}=\bar{\xi}
$$

Therefore, $F_{0}=I$ and

$$
\left.\frac{d}{d t} F_{t}(x)\right|_{t=0}=\xi(x) \text {. }
$$


Definition 2.1. The vector bundle $J^{k} T=\left.V_{\alpha}^{k}\right|_{I}$ on $M$ is the (differentiable) algebroid associated with the groupoid $Q^{k}$.

The Lie bracket $[,]_{k}$ on local sections of $J^{k} T$ is well defined, given by

$$
[\xi, \eta]_{k}=\left.[\bar{\xi}, \bar{\eta}]\right|_{I},
$$

where $\xi, \eta$ are sections of $\pi^{k}: J^{k} T \rightarrow M$ defined on an open set $U$ of $M$ (or $I$ ).

Proposition 2.1. If $f$ is a real function on $U$, and $\xi, \eta$ sections of $J^{k} T$ on $U$, then

$$
[f \xi, \eta]_{k}=f[\xi, \eta]_{k}-\left(\beta_{*} \eta\right)(f) \xi \text {. }
$$

Proof. As $\overline{f \xi}=(f \beta) \bar{\xi}$, it follows

$$
[f \xi, \eta]_{k}=\left.[(f \beta) \bar{\xi}, \bar{\eta}]\right|_{I}=\left.((f \beta)[\bar{\xi}, \bar{\eta}]-\bar{\eta}(f \beta) \bar{\xi})\right|_{I}=f[\xi, \eta]_{k}-\left(\beta_{*} \eta\right)(f) \xi .
$$

If $J^{k} \mathcal{T}$ denotes the sheaf of germs of local sections of $J^{k} T$, then $J^{k} \mathcal{T}$ is a Lie algebra sheaf, with the Lie bracket $[,]_{k}$.

Proposition 2.2. The bracket $[,]_{k}$ on $J^{k} \mathcal{T}$ is determined by:

(i) $\left[j^{k} \xi, j^{k} \eta\right]_{k}=j^{k}[\xi, \eta], \xi, \eta \in \mathcal{T}$,

(ii) $\left[\xi_{k}, f \eta_{k}\right]_{k}=f\left[\xi_{k}, \eta_{k}\right]_{k}+\left(\beta_{*} \xi_{k}\right)(f) \eta_{k}$,

where $\xi_{k}, \eta_{k} \in J^{k} \mathcal{T}$ and $f$ is a real function on $M$.

We denote by the same symbols as above the projections $\pi_{l}^{k}=\pi_{l}: J^{k} T \rightarrow J^{l} T, l \geq 0$, defined by $\pi_{l}\left(j_{x}^{k} \theta\right)=j_{x}^{l} \theta$. If $\xi_{k}$ is a point or a section of $J^{k} T$, let be $\xi_{l}=\pi_{l}^{k}\left(\xi_{k}\right)$. The vector bundle $J^{0} T$ is isomorphic to $T$ by the map $\beta_{*}: J^{0} T \rightarrow T$, where $\beta_{*}\left(j_{x}^{0} \theta\right)=\theta(x)$, see (2.9). However, $\beta_{*}: J^{k} T \rightarrow T$ is not equal to $\pi_{0}^{k}: J^{k} T \rightarrow J^{0} T$, but they are isomorphic maps.

Again, we have the canonical inclusions, and we use the same notation as (2.1),

$$
\begin{aligned}
\lambda^{l}: & J^{k+l} T \rightarrow J^{l} J^{k} T, \\
j_{x}^{k+l} \theta & \mapsto j_{x}^{l} j^{k} \theta .
\end{aligned}
$$

for $\theta \in \mathcal{T}$.

Analogously to the definition of holonomic sections of $Q^{k}$, a section $\xi_{k}$ of $J^{k} T$ is holonomic if there exists $\xi \in \mathcal{T}$ such that $\xi_{k}=j^{k} \xi$. Therefore, if $\xi_{k}$ is holonomic, we have $\xi_{k}=j^{k}\left(\beta_{*} \xi_{k}\right)$.

If $\theta: U \subset M \rightarrow J^{k} T$ is a section, let be $\xi=j_{x}^{1} \theta \in J_{x}^{1} J^{k} T, x \in U$. Then $\xi$ can be identified to the linear application

$$
\begin{aligned}
\xi: & T_{x} \rightarrow T_{\theta(x)} J^{k} T, \\
& v \mapsto \theta_{*}(v) .
\end{aligned}
$$

If $\eta \in J_{x}^{1} J^{k} T$ is given by $\eta=j_{x}^{1} \mu$, with $\mu(x)=\theta(x)$, then $(\pi)_{*}(\eta-\xi) v=0$, and we remember that $\pi: J^{k} T \rightarrow M$ is defined by $\pi\left(j_{x}^{k} \theta\right)=x$. So $\eta-\xi \in T_{x}^{*} \otimes V_{\pi_{0}^{1}(\xi)} J^{k} T$, where $V J^{k} T=\operatorname{ker} \pi_{*}$. However $J^{k} T$ is a vector bundle, then $V_{\pi_{0}^{1} \xi} J^{k} T \cong J_{x}^{k} T$, so $\eta-\xi \in T_{x}^{*} \otimes J_{x}^{k} T$. The sequence

$$
0 \rightarrow T^{*} \otimes J^{k} T \rightarrow J^{1} J^{k} T \stackrel{\pi_{0}^{1}}{\rightarrow} J^{k} T \rightarrow 0
$$

obtained in this way is exact, and we get an affine structure on $J^{1} J^{k} T$. 
The linear operator $D$ defined by

$$
\begin{aligned}
D: & J^{k} \mathcal{T} \rightarrow \mathcal{T}^{*} \otimes J^{k-1} \mathcal{T}, \\
& \xi_{k} \mapsto D \xi_{k}=j^{1} \xi_{k-1}-\lambda^{1}\left(\xi_{k}\right),
\end{aligned}
$$

is the linear Spencer operator. We remember that $\xi_{k-1}=\pi_{k-1}^{k} \xi_{k}$ and

$$
\begin{aligned}
\lambda^{1}: & J^{k} T \\
j_{x}^{k} \xi & \mapsto j_{x}^{1}\left(j^{k-1} \xi\right) .
\end{aligned}
$$

The difference in (2.12) is done in $J^{1} J^{k-1} T$ and is in $T^{*} \otimes J^{k-1} T$, by (2.11).

The operator $D$ is null on a section $\xi_{k}$ if and only if it is holonomic, i.e., $D \xi_{k}=0$ if and only if there exists $\theta \in \mathcal{T}$ such that $\xi_{k}=j^{k} \theta$.

Proposition 2.3. The operator $D$ is characterized by

(i) $D j^{k}=0$,

(ii) $D\left(f \xi_{k}\right)=d f \otimes \xi_{k-1}+f D \xi_{k}$,

with $\xi_{k} \in J^{k} \mathcal{T}, \xi_{k-1}=\pi_{k-1} \xi_{k}$ and $f$ is a real function on $M$.

For a proof, see [14].

The operator $D$ extends to

$$
\begin{aligned}
D: & \wedge^{l} \mathcal{T}^{*} \otimes J^{k} \mathcal{T} \rightarrow \wedge^{l+1} \mathcal{T}^{*} \otimes J^{k-1} \mathcal{T}, \\
& \omega \otimes \xi_{k} \mapsto D\left(\omega \otimes \xi_{k}\right)=d \omega \otimes \xi_{k-1}+(-1)^{l} \omega \wedge D \xi_{k} .
\end{aligned}
$$

\subsection{The calculus on the diagonal}

Next, following $[16,17,14]$, we will relate $\breve{J}^{k} \mathcal{T}$ to vector fields along the diagonal of $M \times M$ and actions of sections in $\mathcal{Q}^{k}$ to diffeomorphisms of $M \times M$ which leave the diagonal invariant.

We denote the diagonal of $M \times M$ by $\Delta=\{(x, x) \in M \times M \mid x \in M\}$, and by $\rho_{1}: M \times M \rightarrow M$ and $\rho_{2}: M \times M \rightarrow M$, the first and second projections, respectively. The restrictions $\left.\rho_{1}\right|_{\Delta}$ and $\left.\rho_{2}\right|_{\Delta}$ are diffeomorphisms of $\Delta$ on $M$. A sheaf on $M$ will be identified to its inverse image by $\left.\rho_{1}\right|_{\Delta}$. For example, if $\mathcal{O}_{M}$ denotes the sheaf of germs of real functions on $M$, then we will write $\mathcal{O}_{M}$ on $\Delta$ instead of $\left(\left.\rho_{1}\right|_{\Delta}\right)^{-1} \mathcal{O}_{M}$. Therefore, a $f \in \mathcal{O}_{M}$ will be considered in $\mathcal{O}_{\Delta}$ or in $\mathcal{O}_{M \times M}$ through the map $f \mapsto f \circ \rho_{1}$.

We denote by $\mathcal{T}(M \times M)$ the sheaf of germs of local sections of $T(M \times M) \rightarrow M \times M$; by $\mathcal{R}$ the subsheaf in Lie algebras of $\mathcal{T}(M \times M)$, whose elements are vector fields $\rho_{1}$-projectables; by $\mathcal{H}_{\mathcal{R}}$ the subsheaf in Lie algebras of $\mathcal{R}$ that projects on 0 by $\rho_{2}$, i.e. $\mathcal{H}_{\mathcal{R}}=\left(\rho_{2}\right)_{*}^{-1}(0) \cap \mathcal{R}$; and by $\mathcal{V}_{\mathcal{R}}$ the subsheaf in Lie algebras defined by $\mathcal{V}_{\mathcal{R}}=\left(\rho_{1}\right)_{*}^{-1}(0) \cap \mathcal{R}$. Clearly,

$$
\mathcal{R}=\mathcal{H}_{\mathcal{R}} \oplus \mathcal{V}_{\mathcal{R}}
$$

and

$$
\left[\mathcal{H}_{\mathcal{R}}, \mathcal{V}_{\mathcal{R}}\right] \subset \mathcal{V}_{\mathcal{R}}
$$

Then

$$
\left(\rho_{1}\right)_{*}: \mathcal{H}_{\mathcal{R}} \simeq \mathcal{T}
$$

is an isomorphism, so we identify $\mathcal{H}_{\mathcal{R}}$ naturally with $\mathcal{T}$ by this isomorphism, and utilize both notations indistinctly. 
Proposition 2.4. The Lie bracket in $\mathcal{R}$ satisfies:

$$
[v+\xi, f(w+\eta)]=v(f)(w+\eta)+f[v+\xi, w+\eta],
$$

with $f \in \mathcal{O}_{M}, v, w \in \mathcal{H}_{\mathcal{R}}, \xi, \eta \in \mathcal{V}_{\mathcal{R}}$. In particular, the Lie bracket in $\mathcal{V}_{\mathcal{R}}$ is $\mathcal{O}_{M}$-bilinear.

Proof. Let be $f \in \mathcal{O}_{M}, \xi, \eta \in \mathcal{V}_{\mathcal{R}}$. Then

$$
\left[v+\xi,\left(f \circ \rho_{1}\right)(w+\eta)\right]=(v+\xi)\left(f \circ \rho_{1}\right)(w+\eta)+\left(f \circ \rho_{1}\right)[v+\xi, w+\eta] .
$$

As $f \circ \rho_{1}$ is constant on the submanifolds $\{x\} \times M$ and $\xi$ is tangent to them, we obtain $\xi\left(f \circ \rho_{1}\right)=0$, and the proposition is proved.

A vector field in $\mathcal{V}_{\mathcal{R}}$ is given by a family of sections of $\mathcal{T}$ parameterized by an open set of $M$. Therefore there exists a surjective morphism

$$
\begin{array}{r}
\Upsilon_{k}: \mathcal{R} \rightarrow \mathcal{T} \oplus J^{k} \mathcal{T}, \\
v+\xi \mapsto v+\xi_{k},
\end{array}
$$

where $v \in \mathcal{H}_{\mathcal{R}}, \xi \in \mathcal{V}_{\mathcal{R}}$, and

$$
\xi_{k}(x)=j_{(x, x)}^{k}\left(\left.\xi\right|_{\{x\} \times M}\right) .
$$

The kernel of morphism $\Upsilon_{k}$ is the subsheaf $\mathcal{V}_{\mathcal{R}}{ }^{k+1}$ of $\mathcal{V}_{\mathcal{R}}$ constituted by vector fields that are null on $\Delta$ at order $k$. Therefore $\mathcal{R} / \mathcal{V}_{\mathcal{R}}{ }^{k+1}$ is null outside $\Delta$. It will be considered as a sheaf on $\Delta$, and the sections in the quotient as sections on open sets of $M$. So the sheaf $\mathcal{R} / \mathcal{V}_{\mathcal{R}}{ }^{k+1}$ is isomorphic to the sheaf os germs of sections of the vector bundle $T \oplus J^{k} T$ on $M$. So we have the isomorphism of sheaves on $M$,

$$
\mathcal{R} / \mathcal{V}_{\mathcal{R}}{ }^{k+1} \equiv \mathcal{T} \oplus J^{k} \mathcal{T}
$$

We usually denote by $\check{J}^{k} T=T \oplus J^{k} T$ and $\check{J}^{k} \mathcal{T}=\mathcal{T} \oplus J^{k} \mathcal{T}$.

As

$$
\left[\mathcal{R}, \mathcal{V}_{\mathcal{R}}{ }^{k+1}\right] \subset \mathcal{V}_{\mathcal{R}}{ }^{k}
$$

the bracket on $\mathcal{R}$ induces a bilinear antisymmetric map, which we call the first bracket of order $k$,

$$
\llbracket, \rrbracket_{k}=\left(\mathcal{T} \oplus J^{k} \mathcal{T}\right) \times\left(\mathcal{T} \oplus J^{k} \mathcal{T}\right) \rightarrow\left(\mathcal{T} \oplus J^{k-1} \mathcal{T}\right)
$$

defined by

$$
\llbracket v+\xi_{k}, w+\eta_{k} \rrbracket_{k}=\Upsilon_{k-1}([v+\xi, w+\eta]),
$$

where $\Upsilon_{k}(\xi)=\xi_{k}$ and $\Upsilon_{k}(\eta)=\eta_{k}$.

It follows from Proposition 2.4 that $\llbracket, \rrbracket_{k}$ satisfies:

$$
\begin{aligned}
& \llbracket v+\xi_{k}, f\left(w+\eta_{k}\right) \rrbracket_{k}=v(f)\left(w+\eta_{k-1}\right)+f \llbracket v+\xi_{k}, w+\eta_{k} \rrbracket_{k}, \\
& \begin{aligned}
\llbracket \llbracket v+\xi_{k}, w+\eta_{k} \rrbracket_{k}, z+\theta_{k-1} \rrbracket_{k-1} & +\llbracket \llbracket w+\eta_{k}, z+\theta_{k} \rrbracket_{k}, v+\xi_{k-1} \rrbracket_{k-1} \\
& +\llbracket \llbracket z+\theta_{k}, v+\xi_{k} \rrbracket_{k}, w+\eta_{k-1} \rrbracket_{k-1}=0,
\end{aligned}
\end{aligned}
$$

for $v, w, z \in \mathcal{T}, \xi_{k}, \eta_{k}, \theta_{k} \in J^{k} \mathcal{T}, f \in \mathcal{O}_{M}$. In particular, the first bracket is $\mathcal{O}_{M}$-bilinear on $J^{k} \mathcal{T}$. Also,

$$
\llbracket J^{0} \mathcal{T}, J^{0} \mathcal{T} \rrbracket_{0}=0 .
$$

The following proposition relates $\llbracket, \rrbracket_{k}$ to the bracket in $\mathcal{T}$ and the linear Spencer operator $D$ in $J^{k} \mathcal{T}$. 
Proposition 2.5. Let be $v, w, \theta, \mu \in \mathcal{T}, \xi_{k}, \eta_{k} \in J^{k} \mathcal{T}$ and $f \in \mathcal{O}_{M}$. Then:

(i) $\llbracket v, w \rrbracket_{k}=[v, w]$, where the bracket at right is the bracket in $\mathcal{T}$;

(ii) $\llbracket v, \xi_{k} \rrbracket_{k}=i(v) D \xi_{k}$;

(iii) $\llbracket j^{k} \theta, j^{k} \mu \rrbracket_{k}=j^{k-1}[\theta, \mu]$, where the bracket at right is the bracket in $\mathcal{T}$.

Proof. (i) This follows from the identification of $\mathcal{T}$ with $\mathcal{H}_{\mathcal{R}}$.

(ii) First of all, if $\theta \in \mathcal{T}$, let be $\Theta \in \mathcal{V}_{\mathcal{R}}$ defined by $\Theta(x, y)=\theta(y)$. Then $\Upsilon_{k}(\Theta)=j^{k} \theta$. If $v \in \mathcal{H}_{\mathcal{R}}$, then $v$ and $\Theta$ are both $\rho_{1}$ and $\rho_{2}$ projectables, $\left(\rho_{1}\right)_{*}(\Theta)=0$ and $\left(\rho_{2}\right)_{*}(v)=0$, so we get $[v, \Theta]=0$. Consequently

$$
\llbracket v, j^{k} \theta \rrbracket_{k}=\Upsilon_{k-1}([v, \Theta])=0 .
$$

Also by (2.14), we have

$$
\llbracket v, f \xi_{k} \rrbracket_{k}=v(f) \xi_{k-1}+f \llbracket v, \xi_{k} \rrbracket_{k} .
$$

As (2.15) and (2.16) determine $D$ (cf. Proposition 2.3), we get (ii).

(iii) Given $\theta, \mu \in \mathcal{T}$, we define $\Theta, H \in \mathcal{V}_{\mathcal{R}}$ as in $(i i), \Theta(x, y)=\theta(y)$ and $H(x, y)=\mu(y)$. Therefore

$$
\llbracket j^{k} \theta, j^{k} \mu \rrbracket_{k}=\llbracket \Upsilon_{k} \Theta, \Upsilon_{k} H \rrbracket_{k}=\Upsilon_{k-1}([\Theta, H])=j^{k-1}[\theta, \eta] .
$$

Let be $\tilde{\mathcal{V}}_{\mathcal{R}}$ the subsheaf in Lie algebras of $\mathcal{R}$ such that $\tilde{\xi} \in \tilde{\mathcal{V}}_{\mathcal{R}}$ if and only if $\tilde{\xi}$ is tangent to the diagonal $\Delta$. If $\tilde{\xi}=\xi_{H}+\xi \in \tilde{\mathcal{V}}_{\mathcal{R}}, \xi_{H} \in \mathcal{H}, \xi \in \mathcal{V}$, then

$$
\left(\rho_{1}\right)_{*}\left(\xi_{H}(x, x)\right)=\left(\rho_{2}\right)_{*}(\xi(x, x)),
$$

where $\rho_{1}, \rho_{2}: M \times M \rightarrow \Delta$. Consequently, if $\xi_{k}=\Upsilon_{k}(\xi)$, then $\xi_{H}=\beta_{*}\left(\xi_{k}\right)$, where we remember that $\beta_{*}: J^{k} T \rightarrow T$ is defined in (2.9). We can also write $\xi_{H}=\left(\rho_{2}\right)_{*}\left(\xi_{k}\right)$, since that $\left.\left(\rho_{2}\right)_{*} \mathcal{V}_{\mathcal{R}}{ }^{k+1}\right|_{\Delta}=0$. From now on, $\xi_{H}$ denotes the horizontal component of $\tilde{\xi} \in \tilde{\mathcal{V}}_{\mathcal{R}}$, so $\tilde{\xi}=\xi_{H}+\xi$, with $\xi_{H} \in \mathcal{H}$ and $\xi \in \mathcal{V}$.

We denote by $J^{k} \tilde{\mathcal{T}}$ the subsheaf of $\mathcal{T} \oplus J^{k} \mathcal{T}=\breve{J}^{k} \mathcal{T}$, whose elements are

$$
\tilde{\xi}_{k}=\xi_{H}+\xi_{k},
$$

where $\xi_{H}=\beta_{*}\left(\xi_{k}\right)$ or $\xi_{H}=\left(\rho_{2}\right)_{*}\left(\xi_{k}\right)$. Therefore $J^{k} \tilde{\mathcal{T}}$ identifies with $\tilde{\mathcal{V}}_{\mathcal{R}} / \mathcal{V}_{\mathcal{R}}{ }^{k+1}$, since that $\mathcal{V}_{\mathcal{R}}{ }^{k+1} \subset \tilde{\mathcal{V}}_{\mathcal{R}}$. As

$$
\left[\tilde{\mathcal{V}}_{\mathcal{R}}, \mathcal{V}_{\mathcal{R}}{ }^{k+1}\right] \subset \mathcal{V}_{\mathcal{R}}{ }^{k+1}
$$

since the vector fields in $\tilde{\mathcal{V}}_{\mathcal{R}}$ are tangents to $\Delta$, it follows that the bracket in $\tilde{\mathcal{V}}_{\mathcal{R}}$ defines a bilinear antisymmetric map, called the second bracket, by

$$
\begin{aligned}
\mathbf{I}, \mathbf{]}_{k}: & J^{k} \tilde{\mathcal{T}} \times J^{k} \tilde{\mathcal{T}} \rightarrow J^{k} \tilde{\mathcal{T}}, \\
& \left(\xi_{H}+\xi_{k}, \eta_{H}+\eta_{k}\right) \mapsto \Upsilon_{k}([\tilde{\xi}, \tilde{\eta}]),
\end{aligned}
$$

where $\Upsilon_{k}(\tilde{\xi})=\xi_{H}+\xi_{k}$ and $\Upsilon_{k}(\tilde{\eta})=\eta_{H}+\eta_{k}$. Unlike the first bracket (2.13), we do not lose one order doing the bracket in $J^{k} \tilde{\mathcal{T}}$. The second bracket $\left\lfloor, \mathbf{l}_{k}\right.$ is a Lie bracket on $J^{k} \tilde{\mathcal{T}}$. The Proposition 2.6 below relates it to the bracket $[,]_{k}$, defined in (2.10).

The projection

$$
\begin{aligned}
\nu: & \mathcal{H}_{\mathcal{R}} \oplus \mathcal{V}_{\mathcal{R}} \rightarrow \mathcal{V}_{\mathcal{R}}, \\
& v+\xi \mapsto \xi
\end{aligned}
$$


quotients to

$$
\begin{aligned}
\nu_{k}: & \mathcal{T} \oplus J^{k} \mathcal{T} \rightarrow J^{k} \mathcal{T}, \\
& v+\xi_{k} \mapsto \xi_{k},
\end{aligned}
$$

and $\nu_{k}: J^{k} \tilde{\mathcal{T}} \rightarrow J^{k} \mathcal{T}$ is an isomorphism of vector bundles.

Proposition 2.6. If $\tilde{\xi}_{k}, \tilde{\eta}_{k} \in J^{k} \tilde{\mathcal{T}}$, then

$$
\left[\xi_{k}, \eta_{k}\right]_{k}=\nu_{k}\left(\mathbf{[} \tilde{\xi}_{k}, \tilde{\eta}_{k} \mathbf{]}_{k}\right)
$$

where $\xi_{k}=\nu_{k}\left(\tilde{\xi}_{k}\right), \eta_{k}=\nu_{k}\left(\tilde{\eta}_{k}\right)$.

Proof. We will verify properties $(i)$ and $(i i)$ of Proposition 2.2. If $\theta, \mu \in \mathcal{T}$, let be $\Theta, H \in \mathcal{V}_{\mathcal{R}}$ as in the proof of Proposition 2.5. Then:

$$
\begin{aligned}
& \text { (i) } \nu_{k}\left(\mathbf{[} \theta+j^{k} \theta, \mu+j^{k} \mu \mathbf{]}_{k}\right)=\nu_{k}\left(\Upsilon_{k}([\theta+\Theta, \mu+H])\right)=\Upsilon_{k}(\nu([\theta, \mu]+[\Theta, H])) \\
& =j^{k}([\theta, \mu])=\left[j^{k} \theta, j^{k} \mu\right]_{k} \text {. }
\end{aligned}
$$

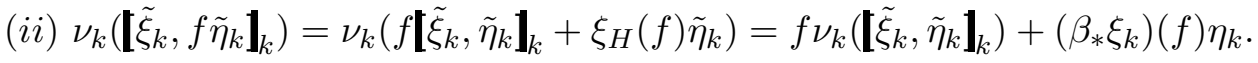

Corollary 2.1. If $\tilde{\xi}_{k}, \tilde{\eta}_{k} \in J^{k} \tilde{\mathcal{T}}$, then

$$
\nu_{k}\left(\tilde{\xi}_{k}, \tilde{\eta}_{k} \mathbf{\rrbracket}_{k}\right)=\left[\xi_{k}, \eta_{k}\right]_{k}=i\left(\xi_{H}\right) D \eta_{k+1}-i\left(\eta_{H}\right) D \xi_{k+1}+\llbracket \xi_{k+1}, \eta_{k+1} \rrbracket_{k+1},
$$

where $\xi_{H}=\beta_{*} \xi_{k}, \eta_{H}=\beta_{*} \eta_{k} \in \mathcal{T}$ and $\xi_{k+1}, \eta_{k+1} \in J^{k+1} \mathcal{T}$ projects on $\xi_{k}, \eta_{k}$, respectively.

Proof. It follows from Propositions 2.5 and 2.6.

As a consequence of Proposition 2.6, we obtain that

$$
\begin{aligned}
\nu_{k}: & J^{k} \tilde{\mathcal{T}} \rightarrow J^{k} \mathcal{T}, \\
& \tilde{\xi}_{k} \mapsto \xi_{k}
\end{aligned}
$$

is an isomorphism of Lie algebras sheaves, where the bracket in $J^{k} \tilde{\mathcal{T}}$ is the second bracket $\mathbf{I}, \mathbf{]}_{k}$ as defined in (2.18), and the bracket in $J^{k} \mathcal{T}$ is the bracket $[,]_{k}$ as defined in (2.10).

In a similar way, we obtain from (2.17) that we can define the third bracket as

$$
\begin{aligned}
\mathbf{I}, \mathbf{I}_{k}: & J^{k+1} \tilde{\mathcal{T}} \times \check{J}^{k} \mathcal{T} \rightarrow \check{J}^{k} \mathcal{T}, \\
& \left(\xi_{H}+\xi_{k+1}, v+\eta_{k}\right) \mapsto \Upsilon_{k}([\tilde{\xi}, v+\eta]) .
\end{aligned}
$$

where $\tilde{\xi} \in \tilde{\mathcal{V}}_{\mathcal{R}}, v+\eta \in \mathcal{R}$,

Proposition 2.7. The third bracket has the following properties:

(i) 【 $f \tilde{\xi}_{k+1}, g \check{\eta}_{k} \mathbf{\coprod}_{k}=f \xi_{H}(g) \check{\eta}_{k}-v(f) g \tilde{\xi}_{k}+f g$ 【 $\tilde{\xi}_{k+1}, \check{\eta}_{k} \mathbf{\coprod}_{k} ;$

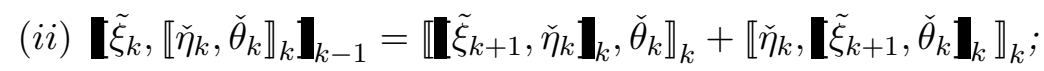

(iii) $\llbracket \tilde{\xi}_{k+1}, \check{\eta}_{k} \rrbracket_{k}=\llbracket \tilde{\xi}_{k+1}, \check{\eta}_{k+1} \rrbracket_{k+1}$,

where $\tilde{\xi}_{k+1}=\xi_{H}+\xi_{k+1} \in \tilde{J}^{k+1} \mathcal{T}, \check{\theta}_{k} \in \check{J}^{k} \mathcal{T}, \check{\eta}_{k+1}=v+\eta_{k+1} \in \check{J}^{k+1} \mathcal{T}, \tilde{\xi}_{k}=\pi_{k}\left(\tilde{\xi}_{k+1}\right)$, $\check{\eta}_{k}=\pi_{k}\left(\check{\eta}_{k+1}\right)$.

Proof. The proof follows the same lines as the proof of Proposition 2.6. 
Let's now verify the relationship between the action of diffeomorphisms of $M \times M$, which are $\rho_{1}$-projectable and preserve $\Delta$, on $\mathcal{R}$, and actions (2.3) and (2.6) of $Q^{1} Q^{k}$ on $T Q^{k}$. Let be $\sigma$ a (local) diffeomorphism of $M \times M$ that is $\rho_{1}$-projectable. Then

$$
\sigma(x, y)=(f(x), \Phi(x, y))
$$

that is, $\sigma$ is defined by $f \in \operatorname{Diff} M$, and a function

$$
\begin{aligned}
\phi: & M \rightarrow \operatorname{Diff} M, \\
& x \mapsto \phi_{x},
\end{aligned}
$$

such that $\phi_{x}(y)=\Phi(x, y)$. Particularly, when $\sigma(\Delta)=\Delta$, then $\Phi(x, x)=f(x)$, for all $x \in M$, or

$$
\phi_{x}(x)=f(x) .
$$

As a special case,

$$
\left(\phi_{f^{-1}(x)}\right)^{-1}(x)=f^{-1}(x) .
$$

Let's denote by $\mathcal{J}$ the set of (local) diffeomorphisms of $M \times M$ that are $\rho_{1}$-projectable and preserve $\Delta$. We naturally have the application

$$
\begin{aligned}
& \mathcal{J} \rightarrow \mathcal{Q}^{k}, \\
& \sigma \mapsto \sigma_{k},
\end{aligned}
$$

where $\sigma_{k}(x)=j_{x}^{k} \phi_{x}, x \in M$. If $\sigma^{\prime} \in \mathcal{J}$, with $\sigma^{\prime}=\left(f^{\prime}, \Phi^{\prime}\right)$, then

$$
\left(\sigma^{\prime} \circ \sigma\right)(x, y)=\sigma^{\prime}\left(f(x), \phi_{x}(y)\right)=\left(f^{\prime}(f(x)), \phi_{f(x)}^{\prime}\left(\phi_{x}(y)\right)=\left(\left(f^{\prime} \circ f\right)(x),\left(\phi_{f(x)}^{\prime} \circ \phi_{x}\right)(y)\right),\right.
$$

and from this it follows

$$
\left(\sigma^{\prime} \circ \sigma\right)_{k}(x)=j_{x}^{k}\left(\phi_{f(x)}^{\prime} \circ \phi_{x}\right)=j_{f(x)}^{k} \phi_{f(x)}^{\prime} \cdot j_{x}^{k} \phi_{x}=\sigma_{k}^{\prime}(f(x)) \cdot \sigma_{k}(x)=\left(\sigma_{k}^{\prime} \circ \sigma_{k}\right)(x),
$$

for each $x \in I$. So (2.19) is a surjective morphism of groupoids. If $\phi \in \operatorname{Diff} M$, let be $\tilde{\phi} \in \mathcal{J}$ given by

$$
\tilde{\phi}(x, y)=(\phi(x), \phi(y)) .
$$

It is clear that

$$
(\tilde{\phi})_{k}=j^{k} \phi
$$

It follows from definitions of $\mathcal{J}$ and $\mathcal{R}$ that the action

$$
\begin{aligned}
& \mathcal{J} \times \mathcal{R} \rightarrow \mathcal{R}, \\
& (\sigma, v+\xi) \mapsto \sigma_{*}(v+\xi),
\end{aligned}
$$

is well defined. Then $\mathcal{V}$ and $\tilde{\mathcal{V}}$ are invariants by the action of $\mathcal{J}$.

Proposition 2.8. Let be $\sigma \in \mathcal{J}, v \in \mathcal{H}_{\mathcal{R}}, \xi \in \mathcal{V}_{\mathcal{R}}$. We have:

(i) $(\sigma * \xi)_{k}=\lambda^{1} \sigma_{k+1} \cdot \xi_{k} \cdot \sigma_{k}^{-1}$;

(ii) $(\sigma * \tilde{\xi})_{k}=f_{*}\left(\xi_{H}\right)+j^{1} \sigma_{k} \cdot \xi_{k} \cdot \sigma_{k}^{-1}$;

(iii) $\left(\sigma_{*} v\right)_{k}=f_{*}(v)+\left(j^{1} \sigma_{k} \cdot v \cdot \lambda^{1} \sigma_{k+1}^{-1}-\lambda^{1} \sigma_{k+1} \cdot v \cdot \lambda^{1} \sigma_{k+1}^{-1}\right)$. 


\section{Proof. If}

$$
\sigma(x, y)=\left(f(x), \phi_{x}(y)\right)
$$

then

$$
\sigma^{-1}(x, y)=\left(f^{-1}(x),\left(\phi^{-1}\right)_{x}(y)\right)
$$

where

$$
\left(\phi^{-1}\right)_{x}=\left(\phi_{f^{-1}(x)}\right)^{-1}
$$

(i) Let be

$$
\xi=\left.\frac{d}{d t} V_{t}\right|_{t=0},
$$

where $V_{t}(x, y)=\left(x, \eta_{x}^{t}(y)\right)$, with $\eta_{x}^{t} \in \operatorname{Diff} M$ for each $t$, and $g_{t}(x)=\eta_{x}^{t}(x)$. Then

$$
\left(\sigma \circ V_{t} \circ \sigma^{-1}\right)(x, y)=\left(x,\left(\phi_{f^{-1}(x)} \circ \eta_{f^{-1}(x)}^{t} \circ\left(\phi_{f^{-1}(x)}\right)^{-1}\right)(y)\right),
$$

and

$$
\left(\sigma_{*} \xi\right)(x, y)=\left.\frac{d}{d t}\left(\phi_{f^{-1}(x)} \circ \eta_{f^{-1}(x)}^{t} \circ\left(\phi_{f^{-1}(x)}\right)^{-1}\right)\right|_{t=0}(y) .
$$

Consequently

$$
\begin{aligned}
\Upsilon_{k}\left(\sigma_{*} \xi\right)(x) & =j_{x}^{k}\left(\left.\frac{d}{d t}\left(\phi_{f^{-1}(x)} \circ \eta_{f^{-1}(x)}^{t} \circ\left(\phi_{f^{-1}(x)}\right)^{-1}\right)\right|_{t=0}\right) \\
& =\left.\frac{d}{d t}\left(j_{\left(g_{t} \circ f^{-1}\right)(x)}^{k} \phi_{f^{-1}(x)} \circ j_{f^{-1}(x)}^{k} \eta_{f^{-1}(x)}^{t} \circ j_{x}^{k}\left(\phi_{f^{-1}(x)}\right)^{-1}\right)\right|_{t=0} \\
& =\left.\frac{d}{d t}\left(\left(\tilde{\phi}_{f^{-1}(x)}\right)_{k}\left(\left(g_{t} \circ f^{-1}\right)(x)\right) \cdot j_{f^{-1}(x)}^{k} \eta_{f^{-1}(x)}^{t} \cdot\left(\left(\tilde{\phi}_{f^{-1}(x)}\right)^{-1}\right)_{k}(x)\right)\right|_{t=0} \\
& =\left.\frac{d}{d t}\left(j_{f^{-1}(x)}^{1}\left(\tilde{\phi}_{f^{-1}(x)}\right)_{k} \cdot j_{f^{-1}(x)}^{k} \eta_{f^{-1}(x)}^{t} \cdot\left(\left(\tilde{\phi}_{f^{-1}(x)}\right)^{-1}\right)_{k}(x)\right)\right|_{t=0} \\
& =\lambda^{1}\left(\sigma_{k+1}\left(f^{-1}(x)\right)\right) \cdot \xi_{k}\left(f^{-1}(x)\right) \cdot \sigma_{k}^{-1}(x),
\end{aligned}
$$

since that

$$
j_{f^{-1}(x)}^{1}\left(\tilde{\phi}_{f^{-1}(x)}\right)_{k}=j_{f^{-1}(x)}^{1} j^{k} \phi_{f^{-1}(x)}=\lambda^{1}\left(j_{f^{-1}(x)}^{k+1} \phi_{f^{-1}(x)}\right)=\lambda^{1}\left(\sigma_{k+1}\left(f^{-1}(x)\right)\right) .
$$

So we proved

$$
(\sigma * \xi)_{k}=\lambda^{1} \sigma_{k+1} \cdot \xi_{k} \cdot \sigma_{k}^{-1}
$$

(ii) Let be, as in $(i), \xi=\left.\frac{d}{d t} V_{t}\right|_{t=0}$, where $V_{t}(x, y)=\left(x, \eta_{x}^{t}(y)\right)$, with $\eta_{x}^{t} \in$ Diff $M$ for each $t$, and $g_{t}(x)=\eta_{x}^{t}(x)$. Then

$$
\tilde{\xi}=\left.\frac{d}{d t} \tilde{V}_{t}\right|_{t=0},
$$

where $\tilde{V}_{t}(x, y)=\left(g_{t}(x), \eta_{x}^{t}(y)\right)$. Therefore

$$
\left(\sigma \circ \tilde{V}_{t} \circ \sigma^{-1}\right)(x, y)=\left(f \circ g_{t} \circ f^{-1}(x),\left(\phi_{g_{t} \circ f^{-1}(x)} \circ \eta_{f^{-1}(x)}^{t} \circ\left(\phi_{f^{-1}(x)}\right)^{-1}\right)(y)\right),
$$


and

$$
\left(\sigma_{*} \tilde{\xi}\right)(x, y)=f_{*} \xi_{H}(x)+\left.\frac{d}{d t}\left(\phi_{g_{t} \circ f^{-1}(x)} \circ \eta_{f^{-1}(x)}^{t} \circ\left(\phi_{f^{-1}(x)}\right)^{-1}\right)\right|_{t=0}(y) .
$$

By projecting, we obtain

$$
\begin{aligned}
\Upsilon_{k}\left(\sigma_{*} \tilde{\xi}\right)(x) & =f_{*} \xi_{H}(x)+j_{x}^{k}\left(\left.\frac{d}{d t}\left(\phi_{g_{t} \circ f^{-1}(x)} \circ \eta_{f^{-1}(x)}^{t} \circ\left(\phi_{f^{-1}(x)}\right)^{-1}\right)\right|_{t=0}\right) \\
& =f_{*} \xi_{H}(x)+\left.\frac{d}{d t}\left(j_{\left(g_{t} \circ f^{-1}\right)(x)}^{k} \phi_{g_{t} \circ f^{-1}(x)} \circ j_{f^{-1}(x)}^{k} \eta_{f^{-1}(x)}^{t} \circ j_{x}^{k}\left(\phi_{f^{-1}(x)}\right)^{-1}\right)\right|_{t=0} \\
& =f_{*} \xi_{H}(x)+\left.\frac{d}{d t}\left(\sigma_{k}\left(\left(g_{t} \circ f^{-1}\right)(x)\right) \cdot j_{f^{-1}(x)}^{k} \eta_{f^{-1}(x)}^{t} \cdot\left(\sigma_{k}\right)^{-1}(x)\right)\right|_{t=0} \\
& =f_{*} \xi_{H}(x)+j_{f^{-1}(x)}^{1} \sigma_{k} \cdot \xi_{k}\left(f^{-1}(x)\right) \cdot \sigma_{k}^{-1}(x),
\end{aligned}
$$

so

$$
\left(\sigma_{*} \tilde{\xi}\right)_{k}=f_{*} \xi_{H}+j^{1} \sigma_{k} \cdot \xi_{k} \cdot \sigma_{k}^{-1}
$$

Observe that this formula depends only on $\sigma_{k}$.

(iii) By combining $(i)$ and $(i i)$, we obtain

$$
\left(\sigma * \xi_{H}\right)_{k}=\left(\sigma_{*} \tilde{\xi}\right)_{k}-(\sigma * \xi)_{k}=\left(f_{*} \xi_{H}+j^{1} \sigma_{k} \cdot \xi_{k} \cdot \sigma_{k}^{-1}\right)-\left(\lambda^{1} \sigma_{k+1} \cdot \xi_{k} \cdot \sigma_{k}^{-1}\right) .
$$

As

$$
\begin{aligned}
j^{1} \sigma_{k} \cdot \xi_{k} \cdot \sigma_{k}^{-1} & =j^{1} \sigma_{k} \cdot \xi_{k} \cdot \lambda^{1} \sigma_{k+1}^{-1}=j^{1} \sigma_{k} \cdot\left(\xi_{k}-\xi_{H}\right) \cdot \lambda^{1} \sigma_{k+1}^{-1}+j^{1} \sigma_{k} \cdot \xi_{H} \cdot \lambda^{1} \sigma_{k+1}^{-1} \\
& =\lambda^{1} \sigma_{k+1} \cdot\left(\xi_{k}-\xi_{H}\right) \cdot \lambda^{1} \sigma_{k+1}^{-1}+j^{1} \sigma_{k} \cdot \xi_{H} \cdot \lambda^{1} \sigma_{k+1}^{-1} \\
& =\lambda^{1} \sigma_{k+1} \cdot \xi_{k} \cdot \sigma_{k}^{-1}-\lambda^{1} \sigma_{k+1} \cdot \xi_{H} \cdot \lambda^{1} \sigma_{k+1}^{-1}+j^{1} \sigma_{k} \cdot \xi_{H} \cdot \lambda^{1} \sigma_{k+1}^{-1}
\end{aligned}
$$

where $j^{1} \sigma_{k} \cdot\left(\xi_{k}-\xi_{H}\right)=\lambda^{1} \sigma_{k+1} \cdot\left(\xi_{k}-\xi_{H}\right)$ follows from $\beta_{*}\left(\xi_{k}-\xi_{H}\right)=0$ by (2.4). By replacing this equality above we get

$$
\begin{aligned}
\left(\sigma * \xi_{H}\right)_{k}= & \left(f_{*} \xi_{H}+\left(\lambda^{1} \sigma_{k+1} \cdot \xi_{k} \cdot \sigma_{k}^{-1}-\lambda^{1} \sigma_{k+1} \cdot \xi_{H} \cdot \lambda^{1} \sigma_{k+1}^{-1}+j^{1} \sigma_{k} \cdot \xi_{H} \cdot \lambda^{1} \sigma_{k+1}^{-1}\right)\right) \\
& -\left(\lambda^{1} \sigma_{k+1} \cdot \xi_{k} \cdot \sigma_{k}^{-1}\right)=f_{*} \xi_{H}-\lambda^{1} \sigma_{k+1} \cdot \xi_{H} \cdot \lambda^{1} \sigma_{k+1}^{-1}+j^{1} \sigma_{k} \cdot \xi_{H} \cdot \lambda^{1} \sigma_{k+1}^{-1} .
\end{aligned}
$$

It follows from Proposition 2.8 that action (2.20) projects on an action ()$_{*}$ :

$$
\begin{aligned}
& \mathcal{Q}^{k+1} \times\left(\mathcal{T} \oplus J^{k} \mathcal{T}\right) \rightarrow \mathcal{T} \oplus J^{k} \mathcal{T}, \\
& \left(\sigma_{k+1}, v+\xi_{k}\right) \mapsto\left(\sigma_{k+1}\right)_{*}\left(v+\xi_{k}\right),
\end{aligned}
$$

where

$$
\left(\sigma_{k+1}\right)_{*}\left(v+\xi_{k}\right)=f_{*} v+\left(j^{1} \sigma_{k} \cdot v \cdot \lambda^{1} \sigma_{k+1}^{-1}-\lambda^{1} \sigma_{k+1} \cdot v \cdot \lambda^{1} \sigma_{k+1}^{-1}\right)+\left(\lambda^{1} \sigma_{k+1} \cdot \xi_{k} \cdot \sigma_{k}^{-1}\right) .
$$

This action verifies

$$
\llbracket\left(\sigma_{k+1}\right)_{*}\left(v+\xi_{k}\right),\left(\sigma_{k+1}\right)_{*}\left(w+\eta_{k}\right) \rrbracket_{k}=\left(\sigma_{k}\right)_{*}\left(\llbracket v+\xi_{k}, w+\eta_{k} \rrbracket_{k}\right) .
$$

It follows from Proposition 2.8 and $(2.21)$ that $\left(\sigma_{k+1}\right)_{*}\left(\xi_{k}\right)(x)$ depends only on the value of $\sigma_{k+1}(x)$ at the point $x$ where $\xi$ is defined, and $\left(\sigma_{k+1}\right)_{*}(v)(x)$ depends on the value of $\sigma_{k+1}$ on a curve tangent to $v(x)$. 
Item $(i i)$ of Proposition 2.8 says that restriction to $J^{k} \tilde{\mathcal{T}}$ of action (2.21) depends only on the section $\sigma_{k}$, so the action

$$
\begin{aligned}
& \mathcal{Q}^{k} \times J^{k} \tilde{\mathcal{T}} \rightarrow J^{k} \tilde{\mathcal{T}}, \\
& \left(\sigma_{k}, \tilde{\xi}_{k}\right) \mapsto\left(\sigma_{k}\right)_{*}\left(\tilde{\xi}_{k}\right),
\end{aligned}
$$

is well defined, where

$$
\left(\sigma_{k}\right)_{*}\left(\tilde{\xi}_{k}\right)=f_{*} \xi_{H}+j^{1} \sigma_{k} \cdot \xi_{k} \cdot \sigma_{k}^{-1} .
$$

In this case, we get

$$
\mathbf{【}\left(\sigma_{k}\right)_{*} \tilde{\xi}_{k},\left(\sigma_{k}\right)_{*} \tilde{\eta}_{k} \mathbf{\rfloor}_{k}=\left(\sigma_{k}\right)_{*}\left[\tilde{\xi}_{k}, \tilde{\eta}_{k} \mathbf{\rfloor}_{k}\right.
$$

and each $\nu_{k}\left(\sigma_{k}\right)_{*} \nu_{k}^{-1}$ acts as an automorphism of the Lie algebra sheaf $J^{k} \mathcal{T}$ :

$$
\left[\nu_{k}\left(\sigma_{k}\right)_{*} \nu_{k}^{-1} \xi_{k}, \nu_{k}\left(\sigma_{k}\right)_{*} \nu_{k}^{-1} \eta_{k}\right]_{k}=\nu_{k}\left(\sigma_{k}\right)_{*} \nu_{k}^{-1}\left[\xi_{k}, \eta_{k}\right]_{k} .
$$

If $M$ and $M^{\prime}$ are two manifolds of the same dimension, we can define

$$
Q^{k}\left(M, M^{\prime}\right)=\left\{j_{x}^{k} f: f: U \subset M \rightarrow U^{\prime} \subset M^{\prime} \text { is a diffeomorphism, } x \in U\right\} .
$$

The groupoid $Q^{k}$ acts by the right on $Q^{k}\left(M, M^{\prime}\right)$, and $Q^{\prime k}=Q^{k}\left(M^{\prime}, M^{\prime}\right)$ acts by the left. Redoing the calculus of this subsection in this context, we obtain the analogous action of (2.21):

$$
\begin{aligned}
& \mathcal{Q}^{k+1}\left(M, M^{\prime}\right) \times\left(\mathcal{T} \oplus J^{k} \mathcal{T}\right) \rightarrow \mathcal{T}^{\prime} \oplus J^{k} \mathcal{T}^{\prime}, \\
& \left(\sigma_{k+1}, v+\xi_{k}\right) \mapsto\left(\sigma_{k+1}\right)_{*}\left(v+\xi_{k}\right),
\end{aligned}
$$

where $\mathcal{Q}^{k+1}\left(M, M^{\prime}\right)$ denotes the set of invertible sections of $Q^{k+1}\left(M, M^{\prime}\right)$,

$$
\left(\sigma_{k+1}\right)_{*}\left(v+\xi_{k}\right)=f_{*} v+\left(j^{1} \sigma_{k} \cdot v \cdot \lambda^{1} \sigma_{k+1}^{-1}-\lambda^{1} \sigma_{k+1} \cdot v \cdot \lambda^{1} \sigma_{k+1}^{-1}\right)+\left(\lambda^{1} \sigma_{k+1} \cdot \xi_{k} \cdot \sigma_{k}^{-1}\right),
$$

and $f=\beta \sigma^{k+1}: M \rightarrow M^{\prime}$. This action also verifies (2.22).

\subsection{The Lie algebra sheaf $\wedge\left(\check{J}^{\infty} \mathcal{T}\right)^{*} \otimes\left(\check{J}^{\infty} \mathcal{T}\right)$}

In this subsection, we continue to follow the presentation of $[16,17]$. We denote by $J^{\infty} \mathcal{T}$ the projective limit of $J^{k} \mathcal{T}$, say,

$$
J^{\infty} \mathcal{T}=\lim \operatorname{proj} J^{k} \mathcal{T}
$$

and

$$
\check{J}^{\infty} \mathcal{T}=\mathcal{T} \oplus J^{\infty} \mathcal{T}=\lim \operatorname{proj} \check{J}^{k} \mathcal{T}
$$

As $\left.T \oplus J^{k} T \cong T Q^{k}\right|_{I}$, we have the identification of $\check{J}^{\infty} T$ with lim proj $\Gamma\left(\left.T Q^{k}\right|_{I}\right)$, where $\Gamma\left(\left.T Q^{k}\right|_{I}\right)$ denotes the sheaf of germs of local sections of the vector bundle $\left.T Q^{k}\right|_{I} \rightarrow I$. From the fact that $\mathcal{T} \oplus J^{k} \mathcal{T}$ is a $\mathcal{O}_{M}$-module, we get $\check{J}^{\infty} \mathcal{T}$ is a $\mathcal{O}_{M}$-module. In the following, we use the notation

$$
\check{\xi}=v+\lim \operatorname{proj} \xi_{k}, \check{\eta}=w+\lim \operatorname{proj} \eta_{k} \in \mathcal{T} \oplus J^{\infty} \mathcal{T} \text {. }
$$

We define the first bracket in $\check{J}^{\infty} \mathcal{T}$ as:

$$
\llbracket \check{\xi}, \check{\eta} \rrbracket_{\infty}=\lim \text { proj } \llbracket v+\xi_{k}, w+\eta_{k} \rrbracket_{k} .
$$


With the bracket defined by $(2.24), \check{J}^{\infty} \mathcal{T}$ is a Lie algebra sheaf. Furthermore,

$$
\llbracket \check{\xi}, f \check{\eta} \rrbracket_{\infty}=v(f) \check{\eta}+f \llbracket \check{\xi}, \check{\eta} \rrbracket_{\infty} .
$$

We extend now, as in $[16,17,14]$, the bracket on $\check{J}^{\infty} \mathcal{T}$ to a Nijenhuis bracket (see [3]) on $\wedge\left(\check{J}^{\infty} \mathcal{T}\right)^{*} \otimes\left(\check{J}^{\infty} \mathcal{T}\right)$, where

$$
\left(\check{J}^{\infty} \mathcal{T}\right)^{*}=\lim \operatorname{ind}\left(\check{J}^{k} \mathcal{T}\right)^{*} \text {. }
$$

We introduce the exterior differential $d$ on $\wedge\left(\check{J}^{\infty} \mathcal{T}\right)^{*}$, by:

(i) if $f \in \mathcal{O}_{M}$, then $d f \in\left(\breve{J}^{\infty} \mathcal{T}\right)^{*}$ is defined by

$$
\langle d f, \check{\xi}\rangle=v(f) .
$$

(ii) if $\omega \in\left(\check{J}^{\infty} \mathcal{T}\right)^{*}$, then $d \omega \in \wedge^{2}\left(\check{J}^{\infty} \mathcal{T}\right)^{*}$ is defined by

$$
\langle d \omega, \check{\xi} \wedge \check{\eta}\rangle=\theta(\check{\xi})\langle\omega, \check{\eta}\rangle-\theta(\check{\eta})\langle\omega, \check{\xi}\rangle-\left\langle\omega, \llbracket \check{\xi}, \check{\eta} \rrbracket_{\infty}\right\rangle,
$$

where $\theta(\check{\xi}) f=\langle d f, \check{\xi}\rangle$.

We extend this operator to forms of any degree as a derivation of degree +1

$$
d: \wedge^{r}\left(\check{J}^{\infty} \mathcal{T}\right)^{*} \rightarrow \wedge^{r+1}\left(\check{J}^{\infty} \mathcal{T}\right)^{*} .
$$

The exterior differential $d$ is linear,

$$
d(\omega \wedge \tau)=d \omega \wedge \tau+(-1)^{r} \omega \wedge d \tau
$$

for $\omega \in \wedge^{r}\left(\check{J}^{\infty} \mathcal{T}\right)^{*}$, and $d^{2}=0$.

Remember that $\left(\rho_{1}\right)_{*}: \mathcal{T} \oplus J^{\infty} \mathcal{T} \rightarrow \mathcal{T}$ is the projection given by the decomposition in direct sum of $\breve{J}^{\infty} \mathcal{T}=\mathcal{T} \oplus J^{\infty} \mathcal{T}$. (We could use, instead of $\left(\rho_{1}\right)_{*}$, the natural map $\alpha_{*}: T \oplus J^{k} T \rightarrow T$, given by $\alpha_{*}:\left.T Q^{k}\right|_{I} \rightarrow T$, and the identification $\left.(2.8)\right)$. Then $\left(\rho_{1}\right)^{*}: \mathcal{T}^{*} \rightarrow\left(\check{J}^{\infty} \mathcal{T}\right)^{*}$, and this map extends to $\left(\rho_{1}\right)^{*}: \wedge \mathcal{T}^{*} \rightarrow \wedge\left(\breve{J}^{\infty} \mathcal{T}\right)^{*}$. If $\omega \in \wedge^{r}\left(\check{J}^{\infty} \mathcal{T}\right)^{*}$, then

$$
\left\langle\left(\rho_{1}\right)^{*} \omega, \check{\xi}_{1} \wedge \cdots \wedge \check{\xi}_{r}\right\rangle=\left\langle\omega, v_{1} \wedge \cdots \wedge v_{r}\right\rangle,
$$

where $\check{\xi}_{j}=v_{j}+\xi_{j}, j=1, \ldots, r$. It follows that $d\left(\left(\rho_{1}\right)^{*} \omega\right)=\left(\rho_{1}\right)^{*}(d \omega)$. We identify $\wedge \mathcal{T}^{*}$ with its image in $\wedge\left(\check{J}^{\infty} \mathcal{T}\right)^{*}$ by $\left(\rho_{1}\right)^{*}$, and we write simply $\omega$ instead of $\left(\rho_{1}\right)^{*} \omega$.

Let be $\mathbf{u}=\omega \otimes \check{\xi} \in \wedge\left(\check{J}^{\infty} \mathcal{T}\right)^{*} \otimes\left(\check{J}^{\infty} \mathcal{T}\right), \tau \in \wedge\left(\check{J}^{\infty} \mathcal{T}\right)^{*}$, with $\operatorname{deg} \omega=r$ and $\operatorname{deg} \tau=s$. We also define $\operatorname{deg} \mathbf{u}=r$. Then we define the derivation of degree $(r-1)$

$$
i(\mathbf{u}): \wedge^{s}\left(\check{J}^{\infty} \mathcal{T}\right)^{*} \rightarrow \wedge^{s+r-1}\left(\check{J}^{\infty} \mathcal{T}\right)^{*}
$$

by

$$
i(\mathbf{u}) \tau=i(\omega \otimes \check{\xi}) \tau=\omega \wedge i(\check{\xi}) \tau
$$

and the Lie derivative

$$
\theta(\mathbf{u}): \wedge^{r}\left(\check{J}^{\infty} \mathcal{T}\right)^{*} \rightarrow \wedge^{r+s}\left(\check{J}^{\infty} \mathcal{T}\right)^{*}
$$

by

$$
\theta(\mathbf{u}) \tau=i(\mathbf{u}) d \tau+(-1)^{r} d(i(\mathbf{u}) \tau),
$$

which is a derivation of degree $r$. If $\mathbf{v}=\tau \otimes \check{\eta}$, we define

$$
[\mathbf{u}, \mathbf{v}]=[\omega \otimes \check{\xi}, \tau \otimes \check{\eta}]=\omega \wedge \tau \otimes \llbracket \check{\xi}, \check{\eta} \rrbracket_{\infty}+\theta(\omega \otimes \check{\xi}) \tau \otimes \check{\eta}-(-1)^{r s} \theta(\tau \otimes \check{\eta}) \omega \otimes \check{\xi}
$$


A straightforward calculation shows that:

$$
[\mathbf{u}, \tau \otimes \check{\eta}]=\theta(\mathbf{u}) \tau \otimes \check{\eta}+(-1)^{r s} \tau \wedge[\mathbf{u}, \check{\eta}]-(-1)^{r s+s} d \tau \wedge i(\check{\eta}) \mathbf{u}
$$

where $i(\check{\eta}) \mathbf{u}=i(\check{\eta})(\omega \otimes \check{\xi})=i(\check{\eta}) \omega \otimes \check{\xi}$. We verify that

$$
[\mathbf{u}, \mathbf{v}]=-(-1)^{r s}[\mathbf{v}, \mathbf{u}]
$$

and

$$
[\mathbf{u},[\mathbf{v}, \mathbf{w}]]=[[\mathbf{u}, \mathbf{v}], \mathbf{w}]+(-1)^{r s}[\mathbf{v},[\mathbf{u}, \mathbf{w}]]
$$

where $\operatorname{deg} \mathbf{u}=r, \operatorname{deg} \mathbf{v}=s$.

With this bracket, $\wedge\left(\check{J}^{\infty} \mathcal{T}\right)^{*} \otimes\left(\check{J}^{\infty} \mathcal{T}\right)$ is a Lie algebra sheaf. Furthermore, if

$$
[\theta(\mathbf{u}), \theta(\mathbf{v})]=\theta(\mathbf{u}) \theta(\mathbf{v})-(-1)^{r s} \theta(\mathbf{v}) \theta(\mathbf{u})
$$

then

$$
[\theta(\mathbf{u}), \theta(\mathbf{v})]=\theta([\mathbf{u}, \mathbf{v}]) .
$$

In particular, we have the following formulas:

Proposition 2.9. If $\mathbf{u}, \mathbf{v} \in \wedge^{1}\left(\check{J}^{\infty} \mathcal{T}\right)^{*} \otimes\left(\check{J}^{\infty} \mathcal{T}\right), \omega \in \wedge^{1}\left(\check{J}^{\infty} \mathcal{T}\right)^{*}, \check{\xi}, \check{\eta} \in \check{J}^{\infty} \mathcal{T}$, then:

(i) $\langle\theta(\mathbf{u}) \omega, \check{\xi} \wedge \check{\eta}\rangle=\theta(i(\check{\xi}) \mathbf{u})\langle\omega, \check{\eta}\rangle-\theta(i(\check{\eta}) \mathbf{u})\langle\omega, \check{\xi}\rangle$

$$
-\left\langle\omega, \llbracket i(\check{\xi}) \mathbf{u}, \check{\eta} \rrbracket_{\infty}+\llbracket \check{\xi}, i(\check{\eta}) \mathbf{u} \rrbracket_{\infty}-i\left(\llbracket \check{\xi}, \check{\eta} \rrbracket_{\infty}\right) \mathbf{u}\right\rangle,
$$

(ii) $i(\check{\xi})[\mathbf{u}, \check{\eta}]=\llbracket i(\check{\xi}) \mathbf{u}, \check{\eta} \rrbracket_{\infty}-i\left(\llbracket \check{\xi}, \check{\eta} \rrbracket_{\infty}\right) \mathbf{u}$,

(iii) $\langle[\mathbf{u}, \mathbf{v}], \check{\xi} \wedge \check{\eta}\rangle=\llbracket i(\check{\xi}) \mathbf{u}, i(\check{\eta}) \mathbf{v} \rrbracket_{\infty}-\llbracket i(\check{\eta}) \mathbf{u}, i(\check{\xi}) \mathbf{v} \rrbracket_{\infty}$

$$
\begin{aligned}
& -i\left(\llbracket i(\check{\xi}) \mathbf{u}, \check{\eta} \rrbracket_{\infty}-\llbracket i(\check{\eta}) \mathbf{u}, \check{\xi} \rrbracket_{\infty}-i\left(\llbracket \check{\xi}, \check{\eta} \rrbracket_{\infty}\right) \mathbf{u}\right) \mathbf{v} \\
& -i\left(\llbracket i(\check{\xi}) \mathbf{v}, \check{\eta} \rrbracket_{\infty}-\llbracket i(\check{\eta}) \mathbf{v}, \check{\xi} \rrbracket_{\infty}-i\left(\llbracket \check{\xi}, \check{\eta} \rrbracket_{\infty}\right) \mathbf{v}\right) \mathbf{u} .
\end{aligned}
$$

Proof. It is a straightforward calculus applying the definitions.

If we define the groupoid

$$
\mathcal{Q}^{\infty}=\lim \operatorname{proj} \mathcal{Q}^{k},
$$

then for $\sigma=\lim \operatorname{proj} \sigma_{k} \in \mathcal{Q}^{\infty}$, we obtain, from (2.21),

$$
\begin{aligned}
\sigma_{*}: & \check{J}^{\infty} \mathcal{T} \rightarrow \check{J}^{\infty} \mathcal{T}, \\
& \xi=v+\lim \operatorname{proj} \xi_{k} \mapsto \sigma_{*} \xi=\lim \operatorname{proj}\left(\sigma_{k+1}\right)_{*}\left(v+\xi_{k}\right),
\end{aligned}
$$

so the action

$$
\begin{aligned}
& \mathcal{Q}^{\infty} \times \check{J}^{\infty} \mathcal{T} \rightarrow \check{J}^{\infty} \mathcal{T}, \\
& (\sigma, \xi) \mapsto \sigma_{*} \xi,
\end{aligned}
$$

is well defined. It follows from (2.22) that $\sigma_{*}: \check{J}^{\infty} \mathcal{T} \rightarrow \breve{J}^{\infty} \mathcal{T}$ is an automorphism of Lie algebra sheaf.

Given $\sigma \in \mathcal{Q}^{\infty}, \sigma$ acts on $\wedge(\check{J} \mathcal{T})^{*}$ :

$$
\begin{aligned}
\sigma^{*}: & \wedge(\check{J} \mathcal{T})^{*} \rightarrow \wedge(\check{J} \mathcal{T})^{*}, \\
& \omega \mapsto \sigma^{*} \omega,
\end{aligned}
$$


where, if $\omega$ is a $r$-form,

$$
\left\langle\sigma^{*} \omega, \check{\xi}_{1} \wedge \cdots \wedge \check{\xi}_{r}\right\rangle=\left\langle\omega, \sigma_{*}^{-1}\left(\check{\xi}_{1}\right) \wedge \cdots \wedge \sigma_{*}^{-1}\left(\check{\xi}_{r}\right)\right\rangle .
$$

Consequently, $\mathcal{Q}^{\infty}$ acts on $\wedge\left(\check{J}^{\infty} \mathcal{T}\right)^{*} \otimes(\check{J} \mathcal{T})$ :

$$
\begin{aligned}
& \mathcal{Q}^{\infty} \times\left(\wedge\left(\check{J}^{\infty} \mathcal{T}\right)^{*} \otimes\left(\check{J}^{\infty} \mathcal{T}\right)\right) \rightarrow \wedge\left(\check{J}^{\infty} \mathcal{T}\right)^{*} \otimes\left(\check{J}^{\infty} \mathcal{T}\right), \\
& (\sigma, \mathbf{u}) \mapsto \sigma_{*} \mathbf{u},
\end{aligned}
$$

where

$$
\sigma_{*} \mathbf{u}=\sigma_{*}(\omega \otimes \check{\xi})=\sigma^{*}(\omega) \otimes \sigma_{*}(\check{\xi}) .
$$

The action of $\sigma_{*}$ is an automorphism of the Lie algebra sheaf $\wedge\left(\check{J}^{\infty} \mathcal{T}\right)^{*} \otimes\left(\check{J}^{\infty} \mathcal{T}\right)$, i.e.,

$$
\left[\sigma_{*} \mathbf{u}, \sigma_{*} \mathbf{v}\right]=\sigma_{*}[\mathbf{u}, \mathbf{v}] .
$$

\subsection{The first non-linear Spencer complex}

In this subsection we will study the subsheaf $\wedge \mathcal{T}^{*} \otimes J^{\infty} \mathcal{T}$ and introduce linear and non-linear Spencer complexes. Principal references are [16, 17, 14].

Proposition 2.10. The sheaf $\wedge \mathcal{T}^{*} \otimes J^{\infty} \mathcal{T}$ is a Lie algebra subsheaf of $\wedge\left(\check{J}^{\infty} \mathcal{T}\right)^{*} \otimes\left(\check{J}^{\infty} \mathcal{T}\right)$, and

$$
[\omega \otimes \xi, \tau \otimes \eta]=\omega \wedge \tau \otimes \llbracket \xi, \eta \rrbracket_{\infty},
$$

where $\omega, \tau \in \wedge \mathcal{T}^{*}, \xi, \eta \in J^{\infty} \mathcal{T}$.

Proof. Let be $\mathbf{u}=\omega \otimes \xi \in \wedge \mathcal{T}^{*} \otimes J^{\infty} \mathcal{T}$. For any $\tau \in \wedge \mathcal{T}^{*}, i(\xi) \tau=0$, then, by applying (2.25) we obtain $i(\mathbf{u}) \tau=0$, and by $(2.26), \theta(\mathbf{u}) \tau=0$. So $(2.27)$ implies $[\omega \otimes \xi, \tau \otimes \eta]=\omega \wedge \tau \otimes \llbracket \xi, \eta \rrbracket_{\infty}$.

Let be the fundamental form

$$
\chi \in\left(\check{J}^{\infty} \mathcal{T}\right)^{*} \otimes\left(\check{J}^{\infty} \mathcal{T}\right)
$$

defined by

$$
i(\check{\xi}) \chi=\left(\rho_{1}\right)_{*}(\check{\xi})=v,
$$

where $\check{\xi}=v+\xi \in \mathcal{T} \oplus J^{\infty} \mathcal{T}$. In another words, $\chi$ is the projection of $\check{J}^{\infty} \mathcal{T}$ on $\mathcal{T}$, parallel to $J^{\infty} \mathcal{T}$.

If $\mathbf{u}=\lim \mathbf{u}_{k}$, we define $D \mathbf{u}=\lim D \mathbf{u}_{k}$.

Proposition 2.11. If $\omega \in \wedge \mathcal{T}^{*}$, and $\mathbf{u} \in \wedge \mathcal{T}^{*} \otimes J^{\infty} \mathcal{T}$, then:

(i) $\theta(\chi) \omega=d \omega$;

(ii) $[\chi, \chi]=0$;

(iii) $[\chi, \mathbf{u}]=D \mathbf{u}$.

Proof. Let be $\check{\xi}=v+\xi, \check{\eta}=w+\eta \in \mathcal{T} \oplus J^{\infty} \mathcal{T}$.

(i) As $\theta(\chi)$ is a derivation of degree 1 , it is enough to prove $(i)$ for 0 -forms $f$ and 1 -forms $\omega \in\left(\check{J}^{\infty} \mathcal{T}\right)^{*}$. From $(2.26)$ we have $\theta(\chi) f=i(\chi) d f=d f$. It follows from Proposition $2.9(i)$ that

$$
\begin{aligned}
\langle\theta(\chi) \omega, \check{\xi} \wedge \check{\eta}\rangle & =\theta(v)\langle\omega, \check{\eta}\rangle-\theta(w)\langle\omega, \check{\xi}\rangle-\left\langle\omega, \llbracket v, \check{\eta} \rrbracket_{\infty}+\llbracket \check{\xi}, w \rrbracket_{\infty}-\chi\left(\llbracket \check{\xi}, \check{\eta} \rrbracket_{\infty}\right)\right\rangle \\
& =\theta(v)\langle\omega, w\rangle-\theta(w)\langle\omega, v\rangle-\langle\omega,[v, w]+[v, w]-[v, w]\rangle=\langle d \omega, \check{\xi} \wedge \check{\eta}\rangle
\end{aligned}
$$


(ii) By applying Proposition 2.9(iii), we obtain

$$
\begin{aligned}
\left\langle\frac{1}{2}[\chi, \chi], \check{\xi} \wedge \check{\eta}\right\rangle & =[v, w]-i\left(\llbracket v, \check{\eta} \rrbracket_{\infty}-\llbracket w, \check{\xi} \rrbracket_{\infty}-\rho_{1} \llbracket \check{\xi}, \check{\eta} \rrbracket_{\infty}\right) \chi \\
& =[v, w]-([v, w]-[w, v]-[v, w])=0 .
\end{aligned}
$$

(iii) It follows from (2.28) that, for $\mathbf{u}=\omega \otimes \xi$,

$$
[\chi, \mathbf{u}]=\theta(\chi) \omega \otimes \xi+(-1)^{r} \omega \wedge[\chi, \xi]-(-1)^{2 r} d \omega \wedge i(\xi) \chi=d \omega \otimes \xi+(-1)^{r} \omega \wedge[\chi, \xi] .
$$

As $D$ is characterized by Proposition 2.3, it is enough to prove $[\chi, \xi]=D \xi$. It follows from Propositions 2.5(ii) and 2.9(ii) that

$$
i(\check{\eta})[\chi, \xi]=\llbracket i(\check{\eta}) \chi, \xi \rrbracket_{\infty}-i\left(\llbracket \check{\eta}, \xi \rrbracket_{\infty}\right) \chi=\llbracket w, \xi \rrbracket_{\infty}=i(\check{\eta}) D \xi .
$$

If $\mathbf{u}, \mathbf{v} \in \wedge \mathcal{T}^{*} \otimes J^{\infty} \mathcal{T}$, with $\operatorname{deg} \mathbf{u}=r, \operatorname{deg} \mathbf{v}=s$, then we get from (2.29) and Proposition $2.11($ iii) that

$$
D[\mathbf{u}, \mathbf{v}]=[D \mathbf{u}, \mathbf{v}]+(-1)^{r}[\mathbf{u}, D \mathbf{v}],
$$

and

$$
[\chi,[\chi, \mathbf{u}]]=[[\chi, \chi], \mathbf{u}]-[\chi,[\chi, \mathbf{u}]]=-[\chi,[\chi, \mathbf{u}]] .
$$

Therefore, $D^{2} \mathbf{u}=0$, or

$$
D^{2}=0 \text {. }
$$

Then the first linear Spencer complex,

$$
0 \rightarrow \mathcal{T} \stackrel{j^{\infty}}{\rightarrow} J^{\infty} \mathcal{T} \stackrel{D}{\rightarrow} \mathcal{T}^{*} \otimes J^{\infty} \mathcal{T} \stackrel{D}{\rightarrow} \wedge^{2} \mathcal{T}^{*} \otimes J^{\infty} \mathcal{T} \stackrel{D}{\rightarrow} \cdots \stackrel{D}{\rightarrow} \wedge^{m} \mathcal{T}^{*} \otimes J^{\infty} \mathcal{T} \rightarrow 0
$$

where $\operatorname{dim} T=m$, is well defined. This complex projects on

$$
0 \rightarrow \mathcal{T} \stackrel{j^{k}}{\rightarrow} J^{k} \mathcal{T} \stackrel{D}{\rightarrow} \mathcal{T}^{*} \otimes J^{k-1} \mathcal{T} \stackrel{D}{\rightarrow} \wedge^{2} \mathcal{T}^{*} \otimes J^{k-2} \mathcal{T} \stackrel{D}{\rightarrow} \cdots \stackrel{D}{\rightarrow} \wedge^{m} \mathcal{T}^{*} \otimes J^{k-m} \mathcal{T} \rightarrow 0
$$

and is exact (see $[16,17,14])$.

Let be $\gamma^{k}$ the kernel of $\pi_{k}: J^{k} T \rightarrow J^{k-1} T$. Denote by $\delta$ the restriction of $D$ to $\gamma^{k}$. It follows from Proposition 2.3(ii) that $\delta$ is $\mathcal{O}_{M^{-}}$linear and $\delta: \gamma^{k} \rightarrow T^{*} \otimes \gamma^{k-1}$. This map is injective, in fact, if $\xi \in \gamma^{k}$, then by $(2.11), \delta \xi=-\lambda^{1}(\xi)$ is injective. As

$$
i(v) D(i(w) D \xi)-i(w) D(i(v) D \xi)-i([v, w]) D \pi_{k-1} \xi=0,
$$

for $v, w \in \mathcal{T}, \xi \in \gamma^{k} \subset J^{k} \mathcal{T}$, we obtain that $\delta$ is symmetric, $i(v) \delta(i(w) \delta \xi)=i(w) \delta(i(v) \delta \xi)$. Observe that we get the map

$$
\iota: \gamma^{k} \rightarrow S^{2} T^{*} \otimes \gamma^{k-2}
$$

defined by $i(v, w) \iota(\xi)=i(w) \delta(i(v) \delta \xi)$, and, if we go on, we obtain the isomorphism

$$
\gamma^{k} \cong S^{k} T^{*} \otimes J^{0} T
$$

where, given a basis $e_{1}, \ldots, e_{m} \in T$ with the dual basis $e^{1}, \ldots, e^{m} \in T^{*}$, we obtain the basis

$$
f_{l}^{k_{1}, k_{2}, \ldots, k_{m}}=\frac{1}{k_{1} ! k_{2} ! \cdots k_{m} !}\left(e^{1}\right)^{k_{1}}\left(e^{2}\right)^{k_{2}} \cdots\left(e^{m}\right)^{k_{m}} \otimes j^{0} e_{l}
$$


of $S^{k} T^{*} \otimes J^{0} T$, where $k_{1}+k_{2}+\cdots+k_{m}=k, k_{1}, \ldots, k_{m} \geq 0$ and $l=1, \ldots, m$. In this basis

$$
\delta\left(f_{l}^{k_{1}, k_{2}, \ldots, k_{m}}\right)=-\sum_{i=1}^{m} e^{i} \otimes f_{l}^{k_{1}, \ldots, k_{i-1}, k_{i}-1, k_{i+1}, \ldots, k_{m}} .
$$

From the linear Spencer complex, we obtain the exact sequence of morphisms of vector bundles

$$
0 \rightarrow \gamma^{k} \stackrel{\delta}{\rightarrow} T^{*} \otimes \gamma^{k-1} \stackrel{\delta}{\rightarrow} \wedge^{2} \mathcal{T}^{*} \otimes \gamma^{k-2} \stackrel{\delta}{\rightarrow} \cdots \stackrel{\delta}{\rightarrow} \wedge^{m} T^{*} \otimes \gamma^{k-m} \rightarrow 0
$$

Let's now introduce the first non-linear Spencer operator $\mathcal{D}$. The "finite" form $\mathcal{D}$ of the linear Spencer operator $D$ is defined by

$$
\mathcal{D} \sigma=\chi-\sigma_{*}^{-1}(\chi)
$$

where $\sigma \in \mathcal{Q}^{\infty}$.

Proposition 2.12. The operator $\mathcal{D}$ take values in $\mathcal{T}^{*} \otimes J^{\infty} \mathcal{T}$, so

$$
\mathcal{D}: \mathcal{Q}^{\infty} \rightarrow \mathcal{T}^{*} \otimes J^{\infty} \mathcal{T}
$$

and

$$
i(v)(\mathcal{D} \sigma)_{k}=\lambda^{1} \sigma_{k+1}^{-1} \cdot j^{1} \sigma_{k} \cdot v-v,
$$

where $\sigma=\lim \operatorname{proj} \sigma_{k} \in \mathcal{Q}^{\infty}$.

Proof. By applying (2.32) and (2.33), it follows that for $\xi \in J^{\infty} \mathcal{T}$,

$$
i(\xi) \mathcal{D} \sigma=i(\xi) \chi-\sigma_{*}^{-1}\left(i\left(\sigma_{*}(\xi)\right) \chi\right)=0,
$$

and for $v \in \mathcal{T}$,

$$
i(v) \mathcal{D} \sigma=i(v) \chi-\sigma_{*}^{-1}\left(i\left(\sigma_{*}(v)\right) \chi\right)=v-\sigma_{*}^{-1}\left(f_{*} v\right),
$$

where $f=\beta \circ \sigma$. By Proposition 2.8(iii),

$$
i(v)(\mathcal{D} \sigma)_{k}=v-\left(f_{*}^{-1}\left(f_{*} v\right)+j^{1} \sigma_{k}^{-1} \cdot f_{*} v \cdot \lambda^{1} \sigma_{k+1}-\lambda^{1} \sigma_{k+1}^{-1} \cdot f_{*} v \cdot \lambda^{1} \sigma_{k+1}\right) .
$$

By posing $v=\left.\frac{d}{d t} x_{t}\right|_{t=0}$, we obtain

$$
j^{1} \sigma_{k} \cdot v \cdot j^{1} \sigma_{k}^{-1}=\left.\frac{d}{d t}\left(\sigma_{k}\left(x_{t}\right) \cdot \sigma_{k}^{-1}\left(f\left(x_{t}\right)\right)\right)\right|_{t=0}=\left.\frac{d}{d t} f\left(x_{t}\right)\right|_{t=0}=f_{*} v
$$

and by replacing (2.39) in (2.38), we get

$$
\begin{aligned}
i(v)(\mathcal{D} \sigma)_{k} & =-j^{1} \sigma_{k}^{-1} \cdot\left(j^{1} \sigma_{k} \cdot v \cdot j^{1} \sigma_{k}^{-1}\right) \cdot \lambda^{1} \sigma_{k+1}+\lambda^{1} \sigma_{k+1}^{-1} \cdot\left(j^{1} \sigma_{k} \cdot v \cdot j^{1} \sigma_{k}^{-1}\right) \cdot \lambda^{1} \sigma_{k+1} \\
& =\left(\lambda^{1} \sigma_{k+1}^{-1} \cdot j^{1} \sigma_{k} \cdot v-v\right) \cdot j^{1} \sigma_{k}^{-1} \cdot \lambda^{1} \sigma_{k+1}=\left(\lambda^{1} \sigma_{k+1}^{-1} \cdot j^{1} \sigma_{k} \cdot v-v\right) \cdot \sigma_{k}^{-1} \cdot \sigma_{k} \\
& =\lambda^{1} \sigma_{k+1}^{-1} \cdot j^{1} \sigma_{k} \cdot v-v
\end{aligned}
$$

since that $\lambda^{1} \sigma_{k+1}^{-1} \cdot j^{1} \sigma_{k} \cdot v-v$ is $\alpha$-vertical (cf. (2.7)).

Corollary 2.2. We have $\mathcal{D} \sigma=0$ if and only if $\sigma=j^{\infty}(\beta \sigma)$, where $\beta: \mathcal{Q}^{\infty} \rightarrow$ Diff $M$. 
Corollary 2.3. If $\sigma_{k+1} \in \mathcal{Q}^{k+1}$, then

$$
\left(\sigma_{k+1}\right)_{*}(v)=f_{*} v+\left(\sigma_{k+1}\right)_{*}\left(i(v) \mathcal{D} \sigma_{k+1}\right),
$$

for $v \in \mathcal{T}$.

Proof. It follows from (2.21) and Proposition 2.12 that

$$
\begin{aligned}
\left(\sigma_{k+1}\right)_{*}\left(i(v) \mathcal{D} \sigma_{k+1}\right) & =\lambda^{1} \sigma_{k+1} \cdot\left(i(v) \mathcal{D} \sigma_{k+1}\right) \cdot \lambda^{1} \sigma_{k+1}^{-1}=\lambda^{1} \sigma_{k+1} \cdot\left(\lambda^{1} \sigma_{k+1}^{-1} \cdot j^{1} \sigma_{k} \cdot v-v\right) \cdot \lambda^{1} \sigma_{k+1}^{-1} \\
& =j^{1} \sigma_{k} \cdot v \cdot \lambda^{1} \sigma_{k+1}^{-1}-\lambda^{1} \sigma_{k+1} \cdot v \cdot \lambda^{1} \sigma_{k+1}^{-1}=\left(\sigma_{k+1}\right)_{*}(v)-f_{*} v
\end{aligned}
$$

Proposition 2.13. The operator $\mathcal{D}$ has the following properties:

(i) If $\sigma, \sigma^{\prime} \in \mathcal{Q}^{\infty}$,

$$
\mathcal{D}\left(\sigma^{\prime} \circ \sigma\right)=\mathcal{D} \sigma+\sigma_{*}^{-1}\left(\mathcal{D} \sigma^{\prime}\right) .
$$

In particular,

$$
\mathcal{D} \sigma^{-1}=-\sigma_{*}(\mathcal{D} \sigma)
$$

(ii) If $\sigma \in \mathcal{Q}^{\infty}, \mathbf{u} \in \wedge \mathcal{T}^{*} \otimes J^{\infty} \mathcal{T}$,

$$
\mathcal{D}\left(\sigma_{*}^{-1} \mathbf{u}\right)=\sigma_{*}^{-1}(D \mathbf{u})+\left[\mathcal{D} \sigma, \sigma_{*}^{-1} \mathbf{u}\right] .
$$

(iii) If $\xi=\left.\frac{d}{d t} \sigma_{t}\right|_{t=0}$, with $\xi \in J^{\infty} \mathcal{T}$, and $\sigma_{t} \in \mathcal{Q}^{\infty}$ is the 1-parameter group associated to $\xi$, then

$$
D \xi=\left.\frac{d}{d t} \mathcal{D} \sigma_{t}\right|_{t=0}
$$

\section{Proof.}

(i) $\mathcal{D}\left(\sigma^{\prime} \circ \sigma\right)=\chi-\sigma_{*}^{-1}(\chi)+\sigma_{*}^{-1}\left(\chi-\left(\sigma^{\prime}\right)_{*}^{-1}(\chi)\right)=\mathcal{D} \sigma+\sigma_{*}^{-1}\left(\mathcal{D} \sigma^{\prime}\right)$.

(ii) $D\left(\sigma_{*}^{-1} \mathbf{u}\right)=\sigma_{*}^{-1}\left[\sigma_{*} \chi, \mathbf{u}\right]=\sigma_{*}^{-1}\left[\chi-\mathcal{D} \sigma^{-1}, \mathbf{u}\right]=\sigma_{*}^{-1}(D \mathbf{u})-\left[\sigma_{*}^{-1}\left(\mathcal{D} \sigma^{-1}\right), \sigma_{*}^{-1} \mathbf{u}\right]$

$$
=\sigma_{*}^{-1}(D \mathbf{u})+\left[\mathcal{D} \sigma, \sigma_{*}^{-1} \mathbf{u}\right] \text {. }
$$

(iii) $\left.\frac{d}{d t} \mathcal{D} \sigma_{t}\right|_{t=0}=-\frac{d}{d t}\left(\sigma_{t}^{-1}\right)_{*}(\chi)=-[\xi, \chi]=D \xi$.

Proposition 2.12 says that $\mathcal{D}$ is projectable:

$$
\begin{aligned}
\mathcal{D}: \mathcal{Q}^{k+1} & \rightarrow \mathcal{T}^{*} \otimes J^{k} \mathcal{T}, \\
\sigma_{k+1} & \mapsto \mathcal{D} \sigma_{k+1},
\end{aligned}
$$

where

$$
i(v) \mathcal{D} \sigma_{k+1}=\lambda^{1} \sigma_{k+1}^{-1} \cdot j^{1} \sigma_{k} \cdot v-v .
$$

It follows from $[\chi, \chi]=0$ that

$$
0=\sigma_{*}^{-1}([\chi, \chi])=\left[\sigma_{*}^{-1}(\chi), \sigma_{*}^{-1}(\chi)\right]=[\chi-\mathcal{D} \sigma, \chi-\mathcal{D} \sigma]=[\mathcal{D} \sigma, \mathcal{D} \sigma]-2 D(\mathcal{D} \sigma),
$$

therefore

$$
D(\mathcal{D} \sigma)-\frac{1}{2}[\mathcal{D} \sigma, \mathcal{D} \sigma]=0
$$


If we define the non-linear operator

$$
\begin{aligned}
\mathcal{D}_{1}: & \mathcal{T}^{*} \otimes J^{\infty} \mathcal{T} \rightarrow \wedge^{2} \mathcal{T}^{*} \otimes J^{\infty} \mathcal{T}, \\
\mathbf{u} & \mapsto D \mathbf{u}-\frac{1}{2}[\mathbf{u}, \mathbf{u}]
\end{aligned}
$$

then we can write $(2.40)$ as

$$
\mathcal{D}_{1} \mathcal{D}=0
$$

The operator $\mathcal{D}_{1}$ projects in order $k$ to

$$
\mathcal{D}_{1}: \mathcal{T}^{*} \otimes J^{k} \mathcal{T} \rightarrow \wedge^{2} \mathcal{T}^{*} \otimes J^{k-1} \mathcal{T}
$$

where

$$
\mathcal{D}_{1} \mathbf{u}=D \mathbf{u}-\frac{1}{2} \llbracket \mathbf{u}, \mathbf{u} \rrbracket_{k} .
$$

Here $\llbracket \mathbf{u}, \mathbf{u} \rrbracket_{k}$ denotes the analogous of formulas (2.27) and (2.28) projected in the order $k$, so that the extension of first bracket makes sense. We will leave the details to the reader.

We define the first non-linear Spencer complex by

$$
1 \rightarrow \operatorname{Diff} M \stackrel{j^{k+1}}{\rightarrow} \mathcal{Q}^{k+1} \stackrel{\mathcal{D}}{\rightarrow} \mathcal{T}^{*} \otimes J^{k} \mathcal{T} \stackrel{\mathcal{D}_{1}}{\rightarrow} \wedge^{2} \mathcal{T}^{*} \otimes J^{k-1} \mathcal{T}
$$

which is exact in $\mathcal{Q}^{k+1}$.

It is possible to define the first nonlinear Spencer complex $\mathcal{D}$ for invertible sections of $Q^{\infty}\left(M, M^{\prime}\right)$ by:

$$
\mathcal{D} \sigma=\chi-\sigma_{*}^{-1}\left(\chi^{\prime}\right)
$$

where $\sigma \in \mathcal{Q}^{\infty}\left(M, M^{\prime}\right)$ and $\chi^{\prime} \in\left(\check{J}^{\infty} \mathcal{T}^{\prime}\right)^{*} \otimes\left(\check{J}^{\infty} \mathcal{T}^{\prime}\right)$ is the fundamental form. The operator $\mathcal{D}$ take values in $\mathcal{T}^{*} \otimes J^{\infty} \mathcal{T}$, so

$$
\mathcal{D}: \mathcal{Q}^{\infty}\left(M, M^{\prime}\right) \rightarrow \mathcal{T}^{*} \otimes J^{\infty} \mathcal{T}
$$

and the same formula of Proposition 2.12 holds:

$$
i(v)(\mathcal{D} \sigma)_{k}=\lambda^{1} \sigma_{k+1}^{-1} \cdot j^{1} \sigma_{k} \cdot v-v,
$$

where $\sigma=\lim \operatorname{proj} \sigma_{k} \in \mathcal{Q}^{\infty}\left(M, M^{\prime}\right)$. Other properties can easily be generalised.

\section{Partial connections}

In this section we will develop the concept of partial connections or partial covariant derivatives associated with the vector bundle $H \oplus J^{k} V$ in the directions of the distribution $V \subset T$. We thank the referee for pointing out that this concept is already in [15, p. 24]. The construction of connections for $J^{k} T$, the transitive case, is in [7].

Let be $V$ an involutive subvector bundle of $T, a \in M$, and $N$ a (local) submanifold of $M$ such that $T_{a} N \oplus V_{a}=T_{a} M$. Then there exists a coordinate system $(x, y)$ in a neighborhood of $a$ such that $a=(0,0)$, the submanifolds given by points with coordinates $x$ constant are integral submanifolds of $V$, and $N$ is given by the submanifold $y=0$. At least locally, we can suppose that the integral manifolds of $V$ are the fibers of a submersion $\rho: M \rightarrow N$. In the coordinates $(x, y)$, we get $\rho(x, y)=(x, 0)$. If we denote by $H$ the subvector bundle of $T$ given by 
vectors tangent to the submanifolds defined by $y$ constant, then $H$ is involutive and $T=H \oplus V$. Also, $T^{*}=H^{*} \oplus V^{*}$. We denote by $\mathcal{H}$ and $\mathcal{V}$ the sheaves of germs of $H$ and $V$, respectively.

We denote by $Q_{V}^{k}$ the subgroupoid of $Q^{k}$ whose elements are the $k$-jets of local diffeomorphisms $h$ of $M$ which are $\rho$-projectable on the identity of $N$. In the coordinates $(x, y), h(x, y)=$ $\left(x, h_{2}(x, y)\right)$. The sheaf of germs of invertible local $\alpha$-sections of $Q_{V}^{k}$ will be denoted by $\mathcal{Q}_{V}^{k}$. The algebroid associated with $Q_{V}^{k}$ is $J^{k} V$, and we denote by $J^{k} \mathcal{V}$ the sheaf of germs of local sections of $J^{k} V$. Then the first non-linear Spencer operator $\mathcal{D}$ can be restricted to $\mathcal{Q}_{V}^{k+1}$,

$$
\mathcal{D}: \mathcal{Q}_{V}^{k+1} \rightarrow \mathcal{T}^{*} \otimes J^{k} \mathcal{V}
$$

and the linear Spencer operator $D$ can be restricted to $J^{k+1} \mathcal{V}$,

$$
D: J^{k+1} \mathcal{V} \rightarrow \mathcal{T}^{*} \otimes J^{k} \mathcal{V}
$$

A vector $u \in T$ decomposes in $u=u_{H}+u_{V}, u_{H} \in H, u_{V} \in V$. If $d$ is the exterior differential, we get the decomposition $d=d_{H}+d_{V}$. The fundamental form $\chi$ decomposes in $\chi=\chi_{H}+\chi_{V}$, where $\chi_{H}(u)=\chi\left(u_{H}\right)$ and $\chi_{V}(u)=\chi\left(u_{V}\right)$. The linear Spencer operator $D$ also decomposes in $D=D_{H}+D_{V}$, and it follows from Proposition 2.11(iii) that

$$
D_{H} \xi=\left[\chi_{H}, \xi\right] \quad \text { and } \quad D_{V} \xi=\left[\chi_{V}, \xi\right],
$$

for $\xi \in J^{k} \mathcal{T}$.

Proposition 3.1. If $\omega \in \wedge \mathcal{V}^{*}$, and $\mathbf{u} \in \wedge \mathcal{V}^{*} \otimes J^{\infty} \mathcal{T}$, then:

(i) $\theta\left(\chi_{V}\right) \omega=d_{V} \omega$;

(ii) $\left[\chi_{V}, \chi_{V}\right]=0$;

(iii) $\left[\chi_{V}, \mathbf{u}\right]=D_{V} \mathbf{u}$.

Proof. Let be $\check{\xi}=v+\xi, \check{\eta}=w+\eta \in \mathcal{T} \oplus J^{\infty} \mathcal{T}, v=v_{H}+v_{V}, w=w_{H}+w_{V} \in \mathcal{H} \oplus \mathcal{V}$.

(i) As $\theta\left(\chi_{V}\right)$ is a derivation of degree 1, it is enough to prove $(i)$ for 0 -forms $f$ and 1 -forms $\omega \in \mathcal{V}^{*}$. From $(2.26)$ we have

$$
\theta\left(\chi_{V}\right) f=i\left(\chi_{V}\right) d f=d_{V} f .
$$

It follows from Proposition 2.9(i) that

$$
\begin{aligned}
\left\langle\theta\left(\chi_{V}\right) \omega, \check{\xi} \wedge \check{\eta}\right\rangle & =\theta\left(v_{V}\right)\langle\omega, \check{\eta}\rangle-\theta\left(w_{V}\right)\langle\omega, \check{\xi}\rangle-\left\langle\omega, \llbracket v_{V}, \check{\eta} \rrbracket_{\infty}+\llbracket \check{\xi}, w_{V} \rrbracket_{\infty}-\chi_{V}\left(\llbracket \check{\xi}, \check{\eta} \rrbracket_{\infty}\right)\right\rangle \\
& =\theta\left(v_{V}\right)\left\langle\omega, w_{V}\right\rangle-\theta\left(w_{V}\right)\left\langle\omega, v_{V}\right\rangle-\left\langle\omega,\left[v_{V}, w\right]_{V}+\left[v, w_{V}\right]_{V}-[v, w]_{V}\right\rangle \\
& =\left\langle d \omega, v_{V} \wedge w_{V}\right\rangle=\left\langle d_{V} \omega, \check{\xi} \wedge \check{\eta}\right\rangle
\end{aligned}
$$

since that

$$
\begin{aligned}
& {\left[v_{V}, w\right]_{V}+\left[v, w_{V}\right]_{V}-[v, w]_{V}} \\
& \quad=\left[v_{V}, w_{V}\right]+\left[v_{V}, w_{H}\right]_{V}+\left[v_{H}, w_{V}\right]_{V}+\left[v_{V}, w_{V}\right]-\left[v_{H}, w_{V}\right]_{V}-\left[v_{V}, w_{H}\right]_{V}-\left[v_{V}, w_{V}\right] \\
& \quad=\left[v_{V}, w_{V}\right] .
\end{aligned}
$$

(ii) By applying Proposition 2.9(iii), we obtain

$$
\begin{aligned}
\left\langle\frac{1}{2}\left[\chi_{V}, \chi_{V}\right], \check{\xi} \wedge \check{\eta}\right\rangle & =\left[v_{V}, w_{V}\right]-i\left(\llbracket v_{V}, \check{\eta} \rrbracket_{\infty}-\llbracket w_{V}, \check{\xi} \rrbracket_{\infty}-\left(\rho_{1}\right)_{*} \llbracket \check{\xi}, \check{\eta} \rrbracket_{\infty}\right) \chi_{V} \\
& =\left[v_{V}, w_{V}\right]-\left(\left[v_{V}, w\right]-\left[w_{V}, v\right]-[v, w]\right)_{V}=0 .
\end{aligned}
$$


(iii) It follows from (2.28) that

$$
\begin{aligned}
{\left[\chi_{V}, \mathbf{u}\right] } & =\theta\left(\chi_{V}\right) \omega \otimes \xi+(-1)^{r} \omega \wedge\left[\chi_{V}, \xi\right]-(-1)^{2 r} d \omega \wedge i(\xi) \chi_{V} \\
& =d_{V} \omega \otimes \xi+(-1)^{r} \omega \wedge\left[\chi_{V}, \xi\right]
\end{aligned}
$$

It is enough to prove $\left[\chi_{V}, \xi\right]=D_{V} \xi$. It follows from Propositions $2.5(i i)$ and $2.9(i i)$ that

$$
i(\check{\eta})\left[\chi_{V}, \xi\right]=\llbracket i(\check{\eta}) \chi_{V}, \xi \rrbracket_{\infty}-i\left(\llbracket \check{\eta}, \xi \rrbracket_{\infty}\right) \chi_{V}=\llbracket w_{V}, \xi \rrbracket_{\infty}=i(\check{\eta}) D_{V} \xi
$$

From item (ii) of this proposition, (2.30) and (2.31) we obtain

$$
d_{V}^{2}=0
$$

The first non-linear Spencer operator $\mathcal{D}$ also decomposes naturally in $\mathcal{D}=\mathcal{D}_{H}+\mathcal{D}_{V}$, where $i(u) \mathcal{D}_{H}=i\left(u_{H}\right) \mathcal{D}$ and $i(u) \mathcal{D}_{V}=i\left(u_{V}\right) \mathcal{D}$. We obtain:

Proposition 3.2. If $F \in \mathcal{Q}_{V}^{k+1}$ is such that $f=\beta F$ satisfies $f_{*} H=H$, then

$$
\mathcal{D}_{H} F=\chi_{H}-F_{*}^{-1}\left(\chi_{H}\right),
$$

and

$$
\mathcal{D}_{V} F=\chi_{V}-F_{*}^{-1}\left(\chi_{V}\right) \text {. }
$$

Proof. From hypothesis, we get $f_{*}\left(u_{H}\right)=\left(f_{*} u\right)_{H}$ and $f_{*}\left(u_{V}\right)=\left(f_{*} u\right)_{V}$, and from equation (2.36), we get

$$
\begin{aligned}
i(u) \mathcal{D}_{H} F & =i\left(u_{H}\right) \mathcal{D} F=i\left(u_{H}\right) \chi-F_{*}^{-1}\left(i\left(f_{*}\left(u_{H}\right)\right) \chi\right)=i\left(u_{H}\right) \chi-F_{*}^{-1}\left(i\left(\left(f_{*} u\right)_{H}\right) \chi\right) \\
& =i(u) \chi_{H}-F_{*}^{-1}\left(i\left(f_{*} u\right) \chi_{H}\right)=i(u)\left(\chi_{H}-F_{*}^{-1}\left(\chi_{H}\right)\right),
\end{aligned}
$$

so $\mathcal{D}_{H} F=\chi_{H}-F_{*}^{-1}\left(\chi_{H}\right)$. The proof of the second formula is analogous.

If we apply Proposition 3.2 to $G F$, we get

$$
\mathcal{D}_{V}(G F)=\chi_{V}-F_{*}^{-1} G_{*}^{-1}\left(\chi_{V}\right)=\chi_{V}+F_{*}^{-1}\left(\mathcal{D}_{V} G-\chi_{V}\right)=\mathcal{D}_{V} F+F_{*}^{-1}\left(\mathcal{D}_{V} G\right),
$$

and if we pose $F=G^{-1}$, we get

$$
\mathcal{D}_{V} G^{-1}=-G_{*}\left(\mathcal{D}_{V} G\right) \text {. }
$$

Definition 3.1. A partial connection $\nabla$ on $H \oplus J^{k} V$ is a $\mathbb{R}$-linear map

$$
\nabla: \mathcal{H} \oplus J^{k} \mathcal{V} \rightarrow \mathcal{V}^{*} \otimes\left(\mathcal{H} \oplus J^{k} \mathcal{V}\right)
$$

such that

$$
\nabla(f \check{\xi})=\left(d_{V} f\right) \otimes \check{\xi}+f \nabla \xi
$$

for $f \in \mathcal{O}_{M}, \check{\xi} \in \mathcal{H} \oplus J^{k} \mathcal{V}$. We extend $\nabla$ to $\wedge V^{*} \otimes\left(H \oplus J^{k} V\right)$ by

$$
\nabla(\alpha \otimes \check{\xi})=d_{V} \alpha \otimes \check{\xi}+(-1)^{|\alpha|} \alpha \wedge \nabla \check{\xi},
$$

where $\alpha \in \wedge \mathcal{V}^{*}$ and $|\alpha|$ is the degree of $\alpha$. 
It follows from $(3.2)$ that $\nabla^{2}(\alpha \otimes \check{\xi})=\alpha \wedge \nabla^{2} \check{\xi}$, so

$$
\nabla^{2}: \mathcal{H} \oplus J^{k} \mathcal{V} \rightarrow \wedge^{2} \mathcal{V}^{*} \otimes\left(\mathcal{H} \oplus J^{k} \mathcal{V}\right)
$$

is a tensor, called the curvature tensor of the partial connection $\nabla$. If $\nabla^{2}=0$ we say that $\nabla$ is flat.

Let be $\omega \in \mathcal{V}^{*} \otimes J^{k+1} \mathcal{V}$ such that $\beta_{*}(i(w) \omega)=w$, for $w \in \mathcal{V}$, and

$$
\tilde{\omega}=\chi_{V}+\omega,
$$

so $\tilde{\omega} \in \mathcal{V}^{*} \otimes J^{k+1} \tilde{\mathcal{V}}$, where $J^{k+1} \tilde{\mathcal{V}}=J^{k+1} \tilde{\mathcal{T}} \cap\left(\mathcal{T} \oplus J^{k+1} \mathcal{V}\right)$.

In the sequel, for $\check{\xi} \in \mathcal{H} \oplus J^{k} \mathcal{V}$, we denote by $\llbracket \tilde{\omega}, \check{\xi} \mathbf{\rfloor}_{k}$ and $\left\lfloor\tilde{\omega}, \tilde{\omega} \mathbf{\rfloor}_{k+1}\right.$ the analogous of formulas (2.27) and (2.28) projected in the order $k$ and $k+1$, respectively, so that the same construction of third and second bracket make sense. We will use this convention in the present section when it makes sense, and leave the details to the reader.

Proposition 3.3. The operator $\nabla$ defined by

$$
\nabla \check{\xi}=\llbracket \tilde{\omega}, \check{\zeta} \mathbf{l}_{k},
$$

for $\tilde{\omega} \in \mathcal{V}^{*} \otimes J^{k+1} \tilde{\mathcal{V}}, \check{\xi}=u+\xi \in \mathcal{H} \oplus J^{k} \mathcal{V}$ is a partial connection on $H \oplus J^{k} V$ with curvature

$$
\left.\nabla^{2}=\frac{1}{2} \llbracket \tilde{\omega}, \tilde{\omega}\right\rfloor_{k+1} .
$$

Proof. If we apply formula (ii) of Proposition 2.9, we obtain

$$
i(\check{\eta}) \llbracket \tilde{\omega}, \check{\xi} \rrbracket_{k}=\llbracket i(\check{\eta}) \tilde{\omega}, \check{\xi} \rrbracket_{k}-i\left(\llbracket \check{\eta}_{k+1}, \check{\xi}_{k+1} \rrbracket_{k+1}\right) \pi_{k} \tilde{\omega}
$$

where $\check{\eta}=v+\eta \in \mathcal{T} \oplus J^{k} \mathcal{T}$, and $\check{\eta}_{k+1}, \check{\xi}_{k+1} \in \mathcal{T} \oplus J^{k+1} \mathcal{T}$ projects on $\check{\eta}, \check{\xi}$, respectively. If $v \in \mathcal{H}$, the right side is 0 , and if $v \in \mathcal{V}$

$$
i(\check{\eta}) \llbracket \tilde{\omega}, \tilde{\xi} \mathbf{\coprod}_{k}=[v, u]_{H}+\llbracket i(v) \tilde{\omega}, \xi \mathbf{\bigsqcup}_{k}-i([v, u]) \pi_{k} \omega-i(u) D(i(v) \omega) \in \mathcal{H} \oplus J^{k} \mathcal{V} .
$$

Then $\nabla \check{\xi} \in \mathcal{V}^{*} \otimes J^{k} \mathcal{V}$. Also,

$$
\begin{aligned}
& i(\check{\eta}) \llbracket \tilde{\omega}, f \check{\xi} \coprod_{k}=\llbracket i(\check{\eta}) \tilde{\omega}, f \check{\xi} \coprod_{k}-i\left(\llbracket \check{\eta}_{k+1}, f \check{\xi}_{k+1} \rrbracket_{k+1}\right) \pi_{k} \tilde{\omega} \\
& =v_{V}(f) \check{\xi}+f \llbracket i(\check{\eta}) \tilde{\omega}, \check{\xi} \rrbracket_{k}-i\left(v(f) \check{\xi}+f \llbracket \check{\eta}_{k+1}, \check{\xi}_{k+1} \rrbracket_{k+1}\right) \pi_{k} \tilde{\omega} \\
& =v_{V}(f) \check{\xi}+f \llbracket i(\check{\eta}) \tilde{\omega}, \check{\xi}_{\mathbf{\beth}_{k}}-i\left(\llbracket \check{\eta}_{k+1}, \check{\xi}_{k+1} \rrbracket_{k+1}\right)\left(f \pi_{k} \tilde{\omega}\right) \\
& =v_{V}(f) \check{\xi}+i(\check{\eta})\left(f \llbracket \tilde{\omega}, \check{\xi} \mathbf{\downarrow}_{k}\right)=i(\check{\eta})\left(d_{V} f\right) \check{\xi}+i(\check{\eta})\left(f \backslash \tilde{\omega}, \check{\xi} \mathbf{\downarrow}_{k}\right) \text {, }
\end{aligned}
$$

so

$$
\nabla(f \check{\xi})=d_{V} f \otimes \check{\xi}+f \nabla \check{\xi} .
$$

If $\alpha \otimes \check{\xi} \in \wedge \mathcal{V}^{*} \otimes\left(\mathcal{H} \oplus J^{k} \mathcal{V}\right)$, we know from (2.28) that

$$
\llbracket \tilde{\omega}, \alpha \otimes \check{\xi} \coprod_{k}=\theta(\tilde{\omega}) \alpha \otimes \check{\xi}+(-1)^{|\alpha|} \alpha \wedge \llbracket \tilde{\omega}, \check{\xi} \coprod_{k}-(-1)^{2|\alpha|} d \alpha \wedge i(\check{\xi}) \tilde{\omega},
$$

and from (2.26) that

$$
\theta(\tilde{\omega}) \alpha=i(\tilde{\omega}) d \alpha-d(i(\tilde{\omega}) \alpha)=i\left(\chi_{V}\right) d \alpha-d\left(i\left(\chi_{V}\right) \alpha\right)=\theta\left(\chi_{V}\right) \alpha=d_{V} \alpha,
$$

and as $i(\check{\xi}) \tilde{\omega}=0$, then

$$
\llbracket \tilde{\omega}, \alpha \otimes \check{\xi} \mathbf{\rrbracket}_{k}=d_{V} \alpha \otimes \check{\xi}+(-1)^{|\alpha|} \alpha \wedge \llbracket \tilde{\omega}, \check{\xi} \rrbracket_{k}=d_{V} \alpha \otimes \check{\xi}+(-1)^{|\alpha|} \alpha \wedge \nabla \check{\xi}
$$


so

$$
\nabla(\alpha \otimes \check{\xi})=\llbracket \tilde{\omega}, \alpha \otimes \check{\xi} \mathbf{\rfloor}_{k}
$$

Therefore,

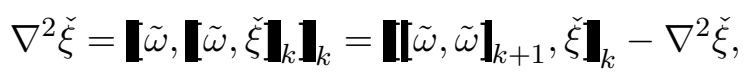

and from this it follows that

$$
\nabla^{2} \check{\xi}=\frac{1}{2} \llbracket \tilde{\omega}, \tilde{\omega} \mathbf{\rfloor}_{k+1}, \check{\xi} \mathbf{\varliminf}_{k}
$$

Let be $\sigma^{y}: N \rightarrow Q_{V}^{k+1}$ a family of differentiable sections such that

$$
\sigma^{y}(x, 0) \in Q_{V}^{k+1}((x, 0),(x, y)),
$$

with $\sigma^{0}(x, 0)=j_{(x, 0)}^{k+1}$ id. Then $\sigma: M \rightarrow Q_{V}^{k+1}$, given by $\sigma(x, y)=\sigma^{y}(x, 0)$, is a differentiable $\beta$-section of $\left.Q_{V}^{k+1}\right|_{N}=\left\{X \in Q_{V}^{k+1}: \alpha(X) \in N\right\}$. If $v(x, y)=\left.\frac{d}{d t}(x, y(t))\right|_{t=0}$, define

$$
\omega \in \mathcal{V}^{*} \otimes J^{k+1} \mathcal{V}
$$

by

$$
i(v(x, y)) \omega=\left.\frac{d}{d t}\left(\sigma^{y(t)}(x, 0) \sigma^{y}(x, 0)^{-1}\right)\right|_{t=0} \in J_{(x, y)}^{k+1} V .
$$

Proposition 3.4. The partial connection $\nabla$ defined by $\tilde{\omega}=\chi_{V}+\omega$, where $\omega$ is defined as in (3.4), is flat.

Proof. First of all,

$$
\beta_{*}(i(v(x, y)) \omega)=\left.\frac{d}{d t} \beta\left(\sigma^{y(t)}(x, 0) \sigma^{y}(x, 0)^{-1}\right)\right|_{t=0}=\left.\frac{d}{d t}(x, y(t))\right|_{t=0}=v(x, y),
$$

so $\omega$ satisfies the condition to define a partial connection. We will show that $\tilde{\omega}$ satisfies $\llbracket \tilde{\omega}, \tilde{\omega}]_{k+1}=0$, i.e., the partial connection $\nabla$ defined by $\tilde{\omega}$ is flat. Let be, for $v \in \mathcal{V}, \overline{i(v) \omega}$ the right invariant vector field defined by

$$
\overline{i(v) \omega}(X)=i(v) \omega(\beta(X)) \cdot X
$$

where $X \in Q_{V}^{k+1}$. We prove that $\overline{i(v) \omega}$ is tangent to $\sigma(M)$, which follows from

$$
\begin{aligned}
\overline{i(v) \omega}(\sigma(x, y)) & =i(v(x, y)) \omega \cdot \sigma(x, y)=\frac{d}{d t}\left[\sigma^{y(t)}(x, 0) \sigma^{y}(x, 0)^{-1}\right]_{t=0} \cdot \sigma(x, y) \\
& =\frac{d}{d t}\left[\sigma^{y(t)}(x, 0) \sigma^{y}(x, 0)^{-1} \sigma(x, y)\right]_{t=0}=\frac{d}{d t}\left[\sigma^{y(t)}(x, 0)\right]_{t=0} \in T(\sigma(M)) .
\end{aligned}
$$

To finish, we know that, for $v, w \in \mathcal{V}, \overline{i(v) \omega}, \overline{i(w) \omega}$ and $\overline{i([v, w]) \omega}$ are tangent to the submanifold $\sigma(M)$, and, as $\beta_{*}([\overline{i(v) \omega}, \overline{i(w) \omega}]=[v, w]$, it follows that

$$
[\overline{i(v) \omega}, \overline{i(w) \omega}]=\overline{i([v, w]) \omega}
$$

so from Proposition 2.6,

$$
\llbracket i(v) \tilde{\omega}, i(w) \tilde{\omega} \mathbf{\varliminf}_{k+1}=i([v, w]) \tilde{\omega} .
$$

From Proposition 2.9(iii) we obtain

$$
\begin{aligned}
i(v \wedge & w)\left(\frac{1}{2}\left\lfloor\tilde{\omega}, \tilde{\omega} \mathbf{\coprod}_{k+1}\right)\right. \\
& =\llbracket i(v) \tilde{\omega}, i(w) \tilde{\omega} \mathbf{\rfloor}_{k+1}-i\left(\llbracket i(v) \tilde{\omega}_{k+2}, w \rrbracket_{k+2}-\llbracket i(w) \tilde{\omega}_{k+2}, v \rrbracket_{k+2}-i([v, w]) \tilde{\omega}\right) \tilde{\omega} \\
& =\llbracket i(v) \tilde{\omega}, i(w) \tilde{\omega} \coprod_{k+1}-i([v, w]-[w, v]-[v, w]) \tilde{\omega}=0,
\end{aligned}
$$

where $\omega_{k+2}$ is a section in $J^{k+2} \mathcal{V}$ that projects on $\omega$. The proposition is proved. 
Therefore, given a section $u+\xi:\left.N \rightarrow\left(H \oplus J^{k} V\right)\right|_{N}$, there exists only one section $(U+\Xi) \in$ $\mathcal{H} \oplus J^{k} \mathcal{V}$ such that $\left.(U+\Xi)\right|_{N}=u+\xi$ and $\nabla(U+\Xi)=0$. The following proposition characterizes these sections:

Proposition 3.5. Let be $(U+\Xi) \in \mathcal{H} \oplus J^{k} \mathcal{V}$ such that

$$
\left.(U+\Xi)\right|_{N}=u+\xi:\left.N \rightarrow\left(H \oplus J^{k} V\right)\right|_{N},
$$

and $\nabla(U+\Xi)=0$. Then

$$
(U+\Xi)(x, y)=\sigma_{*}^{y}((u+\xi)(x, 0)) .
$$

Proof. Choose $v \in \mathcal{V}$ such that $v$ is $H$-projectable, i.e., the 1-parameter group $f_{t}$ of $v$ is given in coordinates by $f_{t}(x, y)=\left(x, h_{t}(y)\right)$. If we define

$$
F_{t}(x, y)=\sigma^{h_{t}(y)}(x, 0) \sigma^{y}(x, 0)^{-1},
$$

then $F_{t} \in Q_{V}^{k+1}$. Furthermore, $\beta F_{t}=f_{t}$, and

$$
\begin{aligned}
\left(F_{s} F_{t}\right)(x, y) & =\left(\sigma^{h_{s}\left(h_{t}(y)\right)}(x, 0) \sigma^{h_{t}(y)}(x, 0)^{-1}\right)\left(\sigma^{h_{t}(y)}(x, 0) \sigma^{y}(x, 0)^{-1}\right) \\
& =\sigma^{h_{s+t}(y)}(x, 0) \sigma^{y}(x, 0)^{-1}=F_{s+t}(x, y) .
\end{aligned}
$$

So, $F_{t}$ is the 1-parameter group such that $\left.\frac{d}{d t} F_{t}\right|_{t=0}=\tilde{\omega}(v)$. If $U+\Xi$ is defined by (3.5), we get

$$
\begin{aligned}
\left(\left(F_{t}\right)_{*}(U+\Xi)\right)(x, y) & =\left(F_{t}\right)_{*}\left((U+\Xi)\left(x, h_{-t}(y)\right)\right)=\left(F_{t}\right)_{*}\left(\sigma_{*}^{h_{-t}(y)}((u+\xi)(x, 0))\right) \\
& =\left(\sigma^{h_{t}\left(h_{-t}(y)\right)}\left(\sigma^{h_{-t}(y)}\right)^{-1}\right)_{*}\left(\sigma^{h_{-t}(y)}\right)_{*}((u+\xi)(x, 0)) \\
& =\sigma_{*}^{y}((u+\xi)(x, 0))=(U+\Xi)(x, y),
\end{aligned}
$$

so $\llbracket(v) \tilde{\omega}, U+\Xi \coprod_{k}=0$. Also,

$$
(U+\Xi)(x, 0)=\sigma_{*}^{0}((u+\xi)(x, 0))=\left(j_{(x, 0)}^{k+1} \mathrm{id}\right)_{*}((u+\xi)(x, 0))=(u+\xi)(x, 0) .
$$

Let $\bar{u} \in \mathcal{H}$ be the vector field $\rho$-projectable such that $\left.\bar{u}\right|_{N}=u$. Then

$$
[\bar{u}, v]=0,
$$

and from (3.5) and Corollary 2.3 we get

$$
U(x, y)=\bar{u}(x, y)+\sigma_{*}^{y}\left(i(u(x, 0)) \mathcal{D} \sigma^{y}\right) \in \mathcal{H} \oplus J^{k} \mathcal{V},
$$

SO

$$
i(v) \nabla(U+\Xi)=\mathbf{\complement} i(v) \tilde{\omega}, U+\Xi \mathbf{\coprod}_{k}-i([v, \bar{u}]) \pi_{k} \tilde{\omega}=0 .
$$

We will now verify how a partial connection defined by $\tilde{\omega}$ changes.

Let $M^{\prime}, V^{\prime}, a^{\prime}, N^{\prime},\left(x^{\prime}, y^{\prime}\right), H^{\prime}, \rho^{\prime}$, be as above, with the same properties and dimensions. Denote by $T^{\prime}=T M^{\prime}$. Let be $\phi: N \rightarrow N^{\prime}$ a (local) diffeomorphism, with $\phi(a)=a^{\prime}$, and denote by $Q_{\phi}^{k}$ the submanifold of $Q^{k}\left(M, M^{\prime}\right)$ of $k$-jets of local diffeomorphisms $\tau: M \rightarrow M^{\prime}$ such that $\rho^{\prime} \tau=\phi \rho$. This means $\tau(x, y)=(\phi(x), b(x, y))$.

Let be $\mathcal{Q}_{\phi}^{k}$ the sheaf of germs of invertible local sections of $Q_{\phi}^{k}$. Then, by restriction of action (2.23), there exists an action of $\mathcal{Q}_{\phi}^{k+1}$ (similar to (2.21)) on $T \oplus J^{k} V$ :

$$
\begin{aligned}
& \mathcal{Q}_{\phi}^{k+1} \times\left(\mathcal{T} \oplus J^{k} \mathcal{V}\right) \rightarrow \mathcal{T}^{\prime} \oplus J^{k} \mathcal{V}^{\prime}, \\
& \left(\sigma_{k+1}, v+\xi_{k}\right) \mapsto\left(\sigma_{k+1}\right)_{*}\left(v+\xi_{k}\right) .
\end{aligned}
$$

The operator $\mathcal{D}: \mathcal{Q}^{k+1}\left(M, M^{\prime}\right) \rightarrow \mathcal{T}^{*} \otimes J^{k} \mathcal{T}$ defined in (2.41) restricts, as (3.1), to

$$
\mathcal{D}: \mathcal{Q}_{\phi}^{k+1} \rightarrow \mathcal{T}^{*} \otimes J^{k} \mathcal{V}
$$

and as above $\mathcal{D}$ decomposes in $\mathcal{D}=\mathcal{D}_{H}+\mathcal{D}_{V}$. The analogous of Proposition 3.2 holds: 
Proposition 3.6. If $F \in \mathcal{Q}_{\phi}^{k+1}$ is such that $f=\beta F$ satisfies $f_{*} H=H^{\prime}$, then

$$
\mathcal{D}_{H} F=\chi_{H}-F_{*}^{-1}\left(\chi_{H^{\prime}}\right),
$$

and

$$
\mathcal{D}_{V} F=\chi_{V}-F_{*}^{-1}\left(\chi_{V^{\prime}}\right) .
$$

Denote by

$$
\mathcal{Q}_{\phi^{-1}}^{k+1}=\left\{\Phi \in \mathcal{Q}^{k+1}\left(M^{\prime}, M\right): \Phi^{-1} \in \mathcal{Q}_{\phi}^{k+1}\right\} .
$$

If $F \in \mathcal{Q}_{\phi}^{k+1}$ satisfy for $f=\beta F, f_{*}(H)=H^{\prime}$, then

$$
\mathcal{D}_{V^{\prime}} F^{-1}=\chi_{V^{\prime}}-F_{*}\left(\chi_{V}\right)
$$

and if $G \in \mathcal{Q}_{\phi^{-1}}^{k+1}$ satisfy $\alpha(G)=\beta(F)$, and for $g=\beta G$ we have $g_{*}\left(H^{\prime}\right)=H$, then by applying Propositions 3.2 and 3.6 to $F G$ we get

$$
\mathcal{D}_{V^{\prime}}(F G)=\chi_{V^{\prime}}-G_{*}^{-1} F_{*}^{-1}\left(\chi_{V^{\prime}}\right)=\chi_{V^{\prime}}+G_{*}^{-1}\left(\mathcal{D}_{V} F-\chi_{V}\right)=\mathcal{D}_{V^{\prime}} G+G_{*}^{-1}\left(\mathcal{D}_{V} F\right) .
$$

By posing $G=F^{-1}$ we get

$$
\mathcal{D}_{V^{\prime}} F^{-1}=-F_{*}\left(\mathcal{D}_{V} F\right) .
$$

Choose $\Phi \in \mathcal{Q}_{\phi}^{k+1}$ with $\varphi=\pi_{0} \Phi$ satisfying $\varphi(N)=N^{\prime}$ and $\varphi_{*}(H)=H^{\prime}$. Then $\left.\varphi\right|_{N}=\phi$ and $\left(x^{\prime}, y^{\prime}\right)=\varphi(x, y)=(\phi(x), b(y))$. Define $\sigma^{\prime y^{\prime}}$ as

$$
\sigma^{\prime y^{\prime}}\left(x^{\prime}, 0\right)=\Phi(x, y) \sigma^{y}(x, 0) \Phi(x, 0)^{-1},
$$

and let be $\omega^{\prime}$ and $\nabla^{\prime}$ as in (3.4) and Proposition 3.3, respectively. Following the proof of Proposition 3.5, take $v \in \mathcal{V}$ such that $v$ is $H$-projectable, i.e., the 1-parameter group $f_{t}$ of $v$ is given in coordinates by $f_{t}(x, y)=\left(x, h_{t}(y)\right)$. Define $F_{t} \in Q_{V}^{k+1}$ by

$$
F_{t}(x, y)=\sigma^{h_{t}(y)}(x, 0) \sigma^{y}(x, 0)^{-1} \text {. }
$$

If $v^{\prime}=\varphi_{*} v$, then $v^{\prime} \in \mathcal{V}^{\prime}$ is $H^{\prime}$-projectable, $f_{t}^{\prime}=\varphi f_{t} \varphi^{-1}$ is the associated 1-parameter group of $v^{\prime}$, and the 1-parameter group associated with $\omega^{\prime}\left(v^{\prime}\right)$ satisfies

$$
\begin{aligned}
F_{t^{\prime}}^{\prime}\left(x^{\prime}, y^{\prime}\right) & =\sigma^{\prime h_{t^{\prime}}\left(y^{\prime}\right)}\left(x^{\prime}, 0\right) \sigma^{y^{\prime}}\left(x^{\prime}, 0\right)^{-1} \\
& =\left(\Phi\left(x, h_{t}(y)\right) \sigma^{h_{t}(y)}(x, 0) \Phi(x, 0)^{-1}\right)\left(\Phi(x, y) \sigma^{y}(x, 0) \Phi(x, 0)^{-1}\right)^{-1} \\
& =\Phi\left(x, h_{t}(y)\right) F_{t}(x, y) \Phi(x, y)^{-1}=\left(\Phi F_{t} \Phi^{-1}\right)\left(x^{\prime}, y^{\prime}\right),
\end{aligned}
$$

i.e., $F_{t}^{\prime}=\Phi F_{t} \Phi^{-1}$. From this, we get $i\left(v^{\prime}\right) \tilde{\omega}^{\prime}=\Phi_{*}(i(v) \tilde{\omega})$, and

$$
i\left(v^{\prime}\right)\left(\Phi_{*} \tilde{\omega}\right)=\Phi_{*}\left(i\left(\Phi_{*}^{-1}\left(v^{\prime}\right)\right) \tilde{\omega}\right)=\Phi_{*}\left(i\left(f_{*}^{-1}\left(v^{\prime}\right)\right) \tilde{\omega}\right)=\Phi_{*}(i(v) \tilde{\omega})=i\left(v^{\prime}\right) \tilde{\omega}^{\prime},
$$

so

$$
\Phi_{*} \tilde{\omega}=\tilde{\omega}^{\prime} .
$$

Then

$$
\nabla^{\prime}\left(\Phi_{*}(U+\Xi)\right)=\llbracket \tilde{\omega}^{\prime}, \Phi_{*}(U+\Xi) \mathbf{\rfloor}_{k}=\Phi_{*} \llbracket \tilde{\omega}, U+\Xi \mathbf{\coprod}_{k},
$$


i.e.,

$$
\nabla^{\prime} \Phi_{*}=\Phi_{*} \nabla
$$

which shows that $\Phi_{*}(U+\Xi)$ is parallel with respect to $\nabla^{\prime}$ if and only if $U+\Xi$ is parallel with respect to $\nabla$.

Taking account of (3.6), the equation (3.9) projected in order $k$ is equivalent to,

$$
\pi_{k}\left(\chi_{V^{\prime}}+\omega^{\prime}\right)=\pi_{k}\left(\Phi_{*}\left(\chi_{V}+\omega\right)\right)=\Phi_{*}\left(\chi_{V}\right)+\Phi_{*}\left(\pi_{k} \omega\right)=\left(\chi_{V^{\prime}}-\mathcal{D}_{V^{\prime}} \Phi^{-1}\right)+\Phi_{*}\left(\pi_{k} \omega\right),
$$

or, considering (3.7),

$$
\chi_{V^{\prime}}+\pi_{k} \omega^{\prime}=\chi_{V^{\prime}}+\Phi_{*}\left(\mathcal{D}_{V} \Phi\right)+\Phi_{*}\left(\pi_{k} \omega\right)
$$

so

$$
\pi_{k} \omega^{\prime}=\Phi_{*}\left(\mathcal{D}_{V} \Phi+\pi_{k} \omega\right)
$$

\section{Linear Lie equations}

Definition 4.1. Let be $R^{k}$ a subvector bundle of $J^{k} T$. We define the prolongation $R^{k+1}$ of $R^{k}$ by

$$
R^{k+1}=\left(\lambda^{1}\right)^{-1}\left(J^{1} R^{k} \cap \lambda^{1}\left(J^{k+1} T\right)\right) \subset J^{k+1} T,
$$

where the intersection is done in $J^{1} J^{k} T$.

We denote the prolongation $\left(R^{k+1}\right)^{+1}$ of $R^{k+1}$ by $R^{k+2}$ and so on, and by $\mathcal{R}^{k+l}$ the sheaf of germs of local sections of $R^{k+l}$, for $l \geq 0$.

Proposition 4.1. A section $\xi \in J^{k+1} \mathcal{T}$ is in $\mathcal{R}^{k+1}$ if and only if $\pi_{k} \xi \in \mathcal{R}^{k}$ and $D \xi \in \mathcal{T}^{*} \otimes \mathcal{R}^{k}$. Proof. From Definition 4.1 and (2.12), since $j^{1} \pi_{k} \xi \in J^{1} \mathcal{R}^{k}$.

Definition 4.2. A subvector bundle $R^{k}$ of $J^{k} T$ is a linear Lie equation if the prolongation $R^{k+1}$ of $R^{k}$ is a subvector bundle of $J^{k+1} T$ such that

(i) $\pi_{k}\left(R^{k+1}\right)=R^{k}$;

(ii) $\llbracket \mathcal{R}^{k+1}, \mathcal{R}^{k+1} \rrbracket_{k+1} \subset \mathcal{R}^{k}$.

It follows from Proposition 4.1 and Definition 4.2 that

$$
\llbracket \mathcal{T} \oplus \mathcal{R}^{k+1}, \mathcal{T} \oplus \mathcal{R}^{k+1} \rrbracket_{k+1} \subset \mathcal{T} \oplus \mathcal{R}^{k},
$$

and from this,

$$
\mathbf{\llbracket} \tilde{\mathcal{R}}^{k}, \tilde{\mathcal{R}}^{k} \mathbf{\rfloor}_{k} \subset \tilde{\mathcal{R}}^{k},
$$

and

$$
\llbracket \tilde{\mathcal{R}}^{k+1}, \mathcal{R}^{k} \mathbf{\rrbracket}_{k} \subset \mathcal{R}^{k} .
$$

Proposition 4.2. If $R^{k} \subset J^{k} T$ is a linear Lie equation, then

$$
\llbracket \mathcal{T} \oplus \mathcal{R}^{k+l}, \mathcal{T} \oplus \mathcal{R}^{k+l} \rrbracket_{k+l} \subset \mathcal{T} \oplus \mathcal{R}^{k+l-1},
$$

for $l \geq 2$. 
Proof. Let's prove this for $l=2$. The other proofs for $l>2$ are equal. Suppose $\xi_{k+2}, \eta_{k+2} \in$ $\mathcal{R}^{k+2}$. Then, by Proposition $4.1, \xi_{k+1}=\pi_{k+1} \xi_{k+2}, \eta_{k+1}=\pi_{k+1} \eta_{k+2} \in \mathcal{R}^{k+1}$, and $D \xi_{k+2}, D \eta_{k+2} \in$ $\mathcal{T} \otimes \mathcal{R}^{k+1}$. So,

$$
\pi_{k} \llbracket \xi_{k+2}, \eta_{k+2} \rrbracket_{k+2}=\llbracket \xi_{k+1}, \eta_{k+1} \rrbracket_{k+1} \in \mathcal{R}^{k},
$$

and

$$
D \llbracket \xi_{k+2}, \eta_{k+2} \rrbracket_{k+2}=\llbracket D \xi_{k+2}, \eta_{k+1} \rrbracket_{k+1}+\llbracket \xi_{k+1}, D \eta_{k+2} \rrbracket_{k+1} \in \mathcal{T}^{*} \otimes \mathcal{R}^{k}
$$

Therefore, by the same Proposition 4.1, $\llbracket \xi_{k+2}, \eta_{k+2} \rrbracket_{k+2} \in \mathcal{R}^{k+1}$, and the proposition follows.

It does not follow from this proposition that $R^{k+l}$ is a vector bundle, and that $\pi_{k+l}: R^{k+l} \rightarrow$ $R^{k+l-1}$ is onto, for $l \geq 2$. To obtain this, we need an additional condition.

Definition 4.3. We say that the linear Lie equation $R^{k}$ is formally integrable if

(i) $R^{k+l}$ is a subvector bundle of $J^{k+l} T$,

(ii) $\pi_{k+l}: R^{k+l+1} \rightarrow R^{k+l}$ is onto,

for $l \geq 1$.

The symbol $g^{k}$ of $R^{k}$ is the kernel of $\pi_{k-1}: R^{k} \rightarrow J^{k-1} T$. Also, $g^{k+l}$ is the kernel of $\pi_{k+l-1}: R^{k+l} \rightarrow R^{k+l-1}$, for $l \geq 1$. It follows from Proposition 4.1 and from (2.35) that we have the subcomplex

$$
0 \rightarrow g^{k+l} \stackrel{\delta}{\rightarrow} T^{*} \otimes g^{k+l-1} \stackrel{\delta}{\rightarrow} \wedge^{2} \mathcal{T}^{*} \otimes g^{k+l-2} \stackrel{\delta}{\rightarrow} \wedge^{3} T^{*} \otimes \gamma^{k+l-3}
$$

for $l \geq 2$.

Definition 4.4. We say that the symbol $g^{k}$ is 2 -acyclic if the subcomplex (4.3) is exact for $l \geq 2$.

The following proposition is in [4, 5]. For an alternative proof, see [21, 22, 23, 26].

Proposition 4.3. If $R^{k} \subset J^{k} T$ is such that

(i) $R^{k+1}$ is a subvector bundle of $J^{k+1} T$,

(ii) $\pi_{k}: R^{k+1} \rightarrow R^{k}$ is onto,

(iii) $g^{k}$ is 2-acyclic,

then $\pi_{k+l-1}: R^{k+l} \rightarrow R^{k+l-1}$ is onto for $l \geq 2$.

A consequence of this proposition is:

Corollary 4.1. If $R^{k} \subset J^{k} T$ is a linear Lie equation and $g^{k}$ is 2-acyclic, then $R^{k}$ is formally integrable.

Given a linear Lie equation $R^{k}$, let be the distribution $B \subset T Q^{k}$ defined by $B_{X}=R_{\beta(X)}^{k} . X$, for $X \in Q^{k}$. It follows from (2.10) and (4.1) that the distribution $B$ is involutive. Let be $P^{k}(x)$ the integral leaf of $B$ that contains the point $I(x)$, and $P^{k}=\cup_{x \in M} P^{k}(x)$. Then $P^{k}$ is a groupoid, and a differentiable submanifold at a neighborhood of $I$. As our problem is local, we will suppose that $P^{k}$ is a differentiable groupoid, the differentiable groupoid associated with the linear Lie equation $R^{k}$. Then the linear Lie equation $R^{k}$ is the Lie algebroid associated with $P^{k}$. As before, we denote by $\mathcal{P}^{k}$ the groupoid of invertible sections of $P^{k}$.

We define the prolongation $P^{k+1}$ of $P^{k}$ by

$$
P^{k+1}=\left(\lambda^{1}\right)^{-1}\left(Q^{1} P^{k} \cap \lambda^{1} Q^{k+1}\right),
$$

where $\lambda^{1}: Q^{k+1} \rightarrow Q^{1} Q^{k}$ and $Q^{1} P^{k}$ is the groupoid of 1 -jets of invertible sections of $P^{k}$. The following is Proposition 6.9(ii) of [17]: 
Proposition 4.4. Let be $F \in \mathcal{Q}^{k+1}$ such that

(i) $\pi_{k} F \in \mathcal{P}^{k}$,

(ii) $\mathcal{D} F \in \mathcal{T}^{*} \otimes \mathcal{R}^{k}$

Then $F \in \mathcal{P}^{k+1}$.

Proof. It follows from $(2.37)$

$$
i(v) \mathcal{D} F=\lambda^{1} F^{-1} \cdot j^{1} \pi_{k} F \cdot v-v,
$$

where $v \in \mathcal{T}$, so

$$
\lambda^{1} F .(i(v) \mathcal{D} F)=j^{1} \pi_{k} F . v-\lambda^{1} F . v .
$$

As $i(v) \mathcal{D} F \in \mathcal{R}^{k}$, we get $\lambda^{1} F .(i(v) \mathcal{D} F) \in \mathcal{T}^{k}$. Also, we get from $\pi_{k} F \in \mathcal{P}^{k}$ that $j^{1} \pi_{k} F . v \in$ $\mathcal{T P}^{k}$. Therefore,

$$
\lambda^{1} F . v=j^{1} \pi_{k} F . v-\lambda^{1} F .(i(v) \mathcal{D} F) \in \mathcal{T} \mathcal{P}^{k},
$$

so $F \in \mathcal{P}^{k+1}$.

If the linear Lie equation $R^{k}$ is formally integrable, and $P^{k}$ is the differentiable groupoid associated with $R^{k}$, it is true (cf. Proposition 6.1, [17]) that the prolongation $P^{k+l}$ of $P^{k}$ is the groupoid associated with the linear Lie equation $R^{k+l}$. Therefore, $\pi_{k+l}: P^{k+l+1} \rightarrow P^{k+l}$ are submersions, for $l \geq 0$.

\section{Formal isomorphism of intransitive linear Lie equations}

In the following sections, we consider intransitive linear Lie equations.

Definition 5.1. We say that a linear Lie equation $R^{k} \subset J^{k} T$ is intransitive if there exists an integrable distribution $V \subset T$ such that $R^{k} \subset J^{k} V$ and $\pi_{0}\left(R^{k}\right)=J^{0} V$.

In reality, considering (4.1), we need only to verify that $\pi_{0}\left(R^{k}\right)$ is a subvector bundle of $J^{0} T$. Our basic problem in this section is to determine the conditions for two intransitive linear Lie equations to be isomorphic. This means that there exists a diffeomorphism that sends one equation onto the other. In the sequel, we give a brief description of the system of partial differential equations that we should solve to obtain a class of diffeomorphisms $f: M \rightarrow M^{\prime}$ such that $\left(j^{k+1} f\right)_{*}\left(R^{k}\right)=R^{\prime k}$. We utilize the same notation of Section 3. Consider $R^{k} \subset J^{k} V$ and $R^{\prime k} \subset J^{k} V^{\prime}$ intransitive linear Lie equations, and $P^{k} \subset Q_{V}^{k}$ and $P^{\prime k} \subset Q_{V^{\prime}}^{k}$ the associated groupoids.

Definition 5.2. We say that a submanifold $S^{k} \subset Q_{\phi}^{k}$ is automorphic by $P^{k}$ if $\alpha: S^{k} \rightarrow M$, $\beta: S^{k} \rightarrow M^{\prime}$ are submersions, and for every $X \in S^{k}(a, b)$, where $a \in M$ and $b \in M^{\prime}$,

$$
S^{k}(\cdot, b)=X \circ P^{k}(\cdot, a) .
$$

We denote by $\mathcal{S}^{k}$ the set of invertible sections of $S^{k}$.

Proposition 5.1. Let $S^{k+1}$ be the prolongation of $S^{k}$. Then an invertible section $F \in \mathcal{Q}_{\phi}^{k+1}$ is such that $F(x) \in S^{k+1}(x)$ for every $x \in \alpha(F)$ if and only if $\pi_{k} F \in \mathcal{S}^{k}$ and $\mathcal{D} F \in \mathcal{T}^{*} \otimes \mathcal{R}^{k}$.

Proof. The same proof of Proposition 4.4 applies. 
We define the symbol

$$
g_{S}^{k}=\left\{v \in T S^{k}:\left(\pi_{k-1}\right)_{*} v=0\right\} .
$$

The symbol $g_{S}^{k}$ of $S^{k}$ is isomorphic to the symbol $g^{k}$ of $R^{k}$, and we get an complex analogous to (4.3), and we define that $g_{S}^{k}$ is 2 -acyclic in the same way. From the formal integrability theorem (see [5]) we obtain:

Proposition 5.2. Let be $S^{k} \subset Q_{\phi}^{k}$ automorphic by $P^{k}$ such that

(i) $S^{k+1}$ is a submanifold of $Q_{\phi}^{k+1}$,

(ii) $\pi_{k}: S^{k+1} \rightarrow S^{k}$ is onto,

(iii) $g_{S}^{k}$ is 2-acyclic.

Then $S^{k}$ is formally integrable, and each prolongation $S^{k+r}$ is automorphic by $P^{k+r}$, for $r \geq 1$.

Definition 5.3. We say that the intransitive linear Lie equation $R^{k} \subset J^{k} V$ is formally isomorphic to the intransitive linear Lie equation $R^{\prime k} \subset J^{k} V^{\prime}$ at points $a$ and $a^{\prime}$, respectively, if there exists a diffeomorphism $\phi: N \rightarrow N^{\prime}$, and a submanifold $S^{k} \subset Q_{\phi}^{k}$ automorphic by $P^{k}$ and formally integrable, such that:

(i) $S^{\prime k}=\left\{X^{-1}: X \in S^{k}\right\} \subset Q_{\phi^{-1}}^{k}$ is automorphic by $P^{\prime k}$;

(ii) $S^{k}\left(a, a^{\prime}\right) \neq \varnothing$.

If there exists a solution $f: M \rightarrow M^{\prime}$ of $S^{k}$, i.e., a diffeomorphism $f$ such that $j^{k} f$ is a section of $S^{k}$, and $f(a)=a^{\prime}$, then $R^{k}$ at point $a$ is said isomorphic to $R^{\prime k}$ at point $a^{\prime}$.

This definition is essentially local. A most useful way to verify the formal isomorphism is given by proposition below, analogous of Proposition 5.1:

Proposition 5.3. Suppose that $R^{k} \subset J^{k} V, R^{\prime k} \subset J^{k} V^{\prime}$ are intransitive linear Lie equations, $N$ and $N^{\prime}$ submanifolds of $M$ and $M^{\prime}$ transversal to integral submanifolds of $V$ and $V^{\prime}$, respectively, and $\phi: N \rightarrow N^{\prime}$ a diffeomorphism, $a \in N, a^{\prime} \in N^{\prime}$, and $\phi(a)=a^{\prime}$. Suppose furthermore that the symbol $g^{k}$ of $R^{k}$ is 2-acyclic. If there exists $F \in \mathcal{Q}_{\phi}^{k+1}$ such that $\left.\beta F\right|_{N}=\phi$, $F_{*}\left(R^{k}\right)=R^{\prime k}$, and $\mathcal{D} F \in \mathcal{T}^{*} \otimes \mathcal{R}^{k}$, then $R^{k}$ at a is formally isomorphic to $R^{\prime k}$ at $a^{\prime}$.

Proof. Define

$$
S^{k+1}=\left\{Y F(x) X: X \in P^{k+1}(\cdot, x), Y \in P^{\prime k+1}(f(x), \cdot), x \in \alpha(F)\right\},
$$

where $f=\beta F: \alpha(F) \subset M \rightarrow \beta(F) \subset M^{\prime}$. Let be $U=\rho^{-1}(\rho(\alpha(F))) \subset M$ and $U^{\prime}=$ $\rho^{\prime-1}\left(\rho^{\prime}(\beta(F))\right) \subset M^{\prime}$. Observe that $S^{k+1} \subset Q_{\phi}^{k+1}$ and $\alpha \times \beta: S^{k+1} \rightarrow U \times U^{\prime}$ is onto (at least locally). If $S^{k}=\pi_{k} S^{k+1}$, then it is a straightforward verification that $S^{k}$ is automorphic by $P^{k}$ and $S^{\prime k}$ is automorphic by $P^{\prime k}$.

Given an invertible section $G \in \mathcal{S}^{k+1}$, then in the neighborhood of each point of $\alpha(G)$, there are invertible sections $G_{1} \in \mathcal{P}^{k+1}$ and $G_{2} \in \mathcal{P}^{\prime k+1}$ such that $G=G_{2} F G_{1}$. In fact, given a point $x \in \alpha(G)$, there is an open set $V_{x} \subset \alpha(G)$, with $x \in V_{x}$, and an invertible section $G_{1}$ of $P^{k+1}$ defined on $V_{x}$, such that $\beta\left(G_{1}\right) \subset \alpha(F)$. Let be $G_{2}=G G_{1}^{-1} F^{-1}$, defined on $f\left(\beta\left(G_{1}\right)\right)$. Then, $G_{2}$ is an invertible section of $P^{\prime k+1}$, and $\left.G\right|_{V_{x}}=G_{2} F G_{1}$. It follows from Proposition 2.13(i) and Proposition 5.1 that $\mathcal{D} G \in \mathcal{T}^{*} \otimes \mathcal{R}^{k}$ on the open set $V_{x}$. As the $V_{x}$ 's cover $\alpha(G)$, we get this property on all $\alpha(G)$. Therefore, $S^{k}$ is formally integrable, and conditions of Definition 5.3 are satisfied. 
Corollary 5.1. Suppose that $R^{k} \subset J^{k} V, R^{\prime k} \subset J^{k} V^{\prime}$ are intransitive linear Lie equations, $N$ and $N^{\prime}$ submanifolds of $M$ and $M^{\prime}$, transversal to integral submanifolds of $V$ and $V^{\prime}$, respectively. Let be $\phi: N \rightarrow N^{\prime}$ a diffeomorphism, $a \in N, a^{\prime} \in N^{\prime}$, and $\phi(a)=a^{\prime}$. Suppose furthermore that the symbol $g^{k}$ of $R^{k}$ is 2-acyclic. If there exists $F \in \mathcal{Q}_{\phi}^{k+1}$ such that $\left.\beta F\right|_{N}=\phi$, and $F_{*}\left(T \oplus R^{k}\right)=T^{\prime} \oplus R^{\prime k}$, then $R^{k}$ at a is formally isomorphic to $R^{\prime k}$ at $a^{\prime}$.

Let's now show the existence of a flat partial connection that leaves $R^{k}$ invariant.

Proposition 5.4. Let be $R^{k} \subset J^{k} V$ an intransitive linear Lie equation. Then there exists a flat partial connection

$$
\nabla: \mathcal{H} \oplus J^{k} \mathcal{V} \rightarrow \mathcal{V}^{*} \otimes\left(\mathcal{H} \oplus J^{k} \mathcal{V}\right)
$$

such that, restricted to $\mathcal{H} \oplus \mathcal{R}^{k}$, it satisfies

$$
\nabla: \mathcal{H} \oplus \mathcal{R}^{k} \rightarrow \mathcal{V}^{*} \otimes\left(\mathcal{H} \oplus \mathcal{R}^{k}\right) .
$$

Furthermore, if $U+\Xi$ is a parallel section of $H \oplus J^{k} V$ and $\left.(U+\Xi)\right|_{N}$ is a section of $\left.\left(H \oplus R^{k}\right)\right|_{N}$, then $U+\Xi \in \mathcal{H} \oplus \mathcal{R}^{k}$.

Proof. Choose a family of differentiable sections $\sigma^{y}$ introduced in (3.3) satisfying $\sigma^{y}(N) \subset$ $P^{k+1}$. As $P^{k+1}$ is the groupoid associated with $R^{k+1}$, the form $\omega$ defined by (3.4) belongs to $\mathcal{V}^{*} \otimes \mathcal{R}^{k+1}$, and the partial connection $\nabla: \mathcal{H} \oplus J^{k} \mathcal{V} \rightarrow \mathcal{V}^{*} \otimes\left(\mathcal{H} \oplus J^{k} \mathcal{V}\right)$ defined by $\tilde{\omega}$, restricted to $H \oplus R^{k}$ sends $\mathcal{H} \oplus \mathcal{R}^{k}$ to $\mathcal{V}^{*} \otimes\left(\mathcal{H} \oplus \mathcal{R}^{k}\right)$, as a consequence of (4.2). The proof now follows from Propositions 3.4 and 3.5.

Now we prove the fundamental theorem for formal isomorphism of linear Lie equations:

Theorem 5.1. Suppose that $R^{k} \subset J^{k} V$ and $R^{\prime k} \subset J^{k} V^{\prime}$ are intransitive linear Lie equations, $N$ and $N^{\prime}$ submanifolds of $M$ and $M^{\prime}$ transversal to integral submanifolds of $V$ and $V^{\prime}$, respectively, and $\phi: N \rightarrow N^{\prime}$ a diffeomorphism. Suppose furthermore that there exists $\Phi: N \rightarrow \mathcal{Q}_{\phi}^{k+1}$ such that $\beta \Phi=\phi$, and

$$
\Phi_{*}\left(\left.T N \oplus R^{k}\right|_{N}\right)=\left.T N^{\prime} \oplus R^{\prime k}\right|_{N^{\prime}}
$$

Then given a diffeomorphism $f: M \rightarrow M^{\prime}$ such that $f_{*} V=V^{\prime},\left.f\right|_{N}=\phi$, there exists $F \in \mathcal{Q}_{\phi}^{k+1}$ satisfying $\left.F\right|_{N}=\Phi, \beta F=f$, and

$$
F_{*}\left(T \oplus R^{k}\right)=T^{\prime} \oplus R^{\prime k} .
$$

Proof. Let be families $\sigma^{y}: N \rightarrow P^{k+1}, \sigma^{\prime y^{\prime}}: N^{\prime} \rightarrow P^{\prime k+1}$, as in the proof of Proposition 5.4, and defining flat partial connections

$$
\nabla: \mathcal{H} \oplus J^{k} \mathcal{V} \rightarrow \mathcal{V}^{*} \otimes\left(\mathcal{H} \oplus J^{k} \mathcal{V}\right)
$$

and

$$
\nabla^{\prime}: \mathcal{H}^{\prime} \oplus J^{k} \mathcal{V}^{\prime} \rightarrow \mathcal{V}^{\prime *} \otimes\left(\mathcal{H}^{\prime} \oplus J^{k} \mathcal{V}^{\prime}\right)
$$

such that

$$
\nabla\left(\mathcal{H} \oplus \mathcal{R}^{k}\right) \subset \mathcal{V}^{*} \otimes\left(\mathcal{H} \oplus \mathcal{R}^{k}\right)
$$

and

$$
\nabla^{\prime}\left(\mathcal{H}^{\prime} \oplus \mathcal{R}^{\prime k}\right) \subset \mathcal{V}^{\prime *} \otimes\left(\mathcal{H}^{\prime} \oplus \mathcal{R}^{\prime k}\right) .
$$


Observe that by Proposition 5.4, $\omega \in \mathcal{V}^{*} \otimes \mathcal{R}^{k+1}$, and $\omega^{\prime} \in \mathcal{V}^{\prime *} \otimes \mathcal{R}^{\prime k+1}$. Redefine $H^{\prime}=f_{*} H$, if necessary, to obtain $\left(x^{\prime}, y^{\prime}\right)=f(x, y)=(a(x), b(y))$, and define $F \in \mathcal{Q}_{\phi}^{k+1}$ by

$$
F(x, y)=\sigma^{\prime y^{\prime}}\left(x^{\prime}, 0\right) \Phi(x, 0) \sigma^{y}(x, 0)^{-1} .
$$

Then, we get from (3.8)

$$
F(x, y) \sigma^{y}(x, 0) F(x, 0)^{-1}=\sigma^{\prime y^{\prime}}\left(x^{\prime}, 0\right),
$$

and from (3.10) we get $\nabla^{\prime} F_{*}=F_{*} \nabla$. By hypothesis

$$
F_{*}\left(\left.T N \oplus R^{k}\right|_{N}\right)=\left.T N^{\prime} \oplus R^{\prime k}\right|_{N^{\prime}}
$$

then by Proposition 5.4 we obtain

$$
F_{*}\left(H \oplus R^{k}\right)=H^{\prime} \oplus R^{\prime k} .
$$

From this and Corollary 2.3 we obtain

$$
\mathcal{D}_{H} F \in \mathcal{H}^{*} \otimes \mathcal{R}^{k}
$$

It follows from (3.11) that

$$
\pi_{k} \omega^{\prime}=F_{*}\left(\mathcal{D}_{V} F+\pi_{k} \omega\right) .
$$

So, from $\pi_{k} \omega \in \mathcal{R}^{k}, \pi_{k} \omega^{\prime} \in \mathcal{R}^{\prime k}$ and (5.1) we get

$$
\mathcal{D}_{V} F \in \mathcal{V}^{*} \otimes \mathcal{R}^{k} \text {. }
$$

Combining this with (5.1) and (5.2), we get $\mathcal{D} F \in \mathcal{T}^{*} \otimes \mathcal{R}^{k}$ and $F_{*}\left(R^{k}\right)=R^{\prime k}$, and by Proposition 5.3 the theorem follows.

Corollary 5.2. Suppose that $R^{k} \subset J^{k} V$ and $R^{\prime k} \subset J^{k} V^{\prime}$ are intransitive linear Lie equations, that $N$ and $N^{\prime}$ are submanifolds of $M$ and $M^{\prime}$ transversal to integral submanifolds of $V$ and $V^{\prime}$, respectively. Let be $\phi: N \rightarrow N^{\prime}$ a diffeomorphism such that $\phi(a)=a^{\prime}$, where $a \in N$ and $a^{\prime} \in N^{\prime}$. Suppose furthermore that the symbol $g^{k}$ of $R^{k}$ is 2-acyclic. If there exists $\Phi: N \rightarrow \mathcal{Q}_{\phi}^{k+1}$ such that $\beta \Phi=\phi$, and $\Phi_{*}\left(\left.T N \oplus R^{k}\right|_{N}\right)=\left.T N^{\prime} \oplus R^{\prime k}\right|_{N^{\prime}}$, then $R^{k}$ at point a is formally isomorphic to $R^{\prime k}$ at point $a^{\prime}$.

Proof. The corollary follows from Theorem 5.1 and Corollary 5.1.

\section{Intransitive Lie algebras}

In this section, we associate an intransitive Lie algebra with a germ of an intransitive linear Lie equation. This definition must generalize the definition of transitive Lie algebra, and incorporate the fact that we can reconstruct an intransitive linear Lie equation from its restriction to a transversal to the orbits, unless of formal isomorphism, as the Theorem of [25] and Theorem 5.1 above shows.

We continue, in this section, to suppose that $R^{k} \subset J^{k} V$ is an intransitive linear Lie equation and $g^{k}$ is 2-acyclic. We remember that it follows from these hypotheses, see Corollary 4.1, that the prolongations $R^{k+l}$ of $R^{k}, l \geq 1$, satisfy:

(i) $R^{k+l}$ is a subvector bundle of $J^{k+l} V$;

(ii) $\pi_{k+l}: R^{k+l+1} \rightarrow R^{k+l}$ is onto; 
(iii) $\llbracket \mathcal{T} \oplus \mathcal{R}^{k+l}, \mathcal{T} \oplus \mathcal{R}^{k+l} \rrbracket_{k+l} \subset \mathcal{T} \oplus \mathcal{R}^{k+l-1}$.

We also assume that $R^{l}=\pi_{l} R^{k}$ is a subvector bundle of $J^{l} V$ for every $0 \leq l \leq k-1$, in particular, $R^{0}=J^{0} V$.

We denote by $\mathcal{O}_{N, a}$ the $\mathbb{R}$-algebra of germs at point $a \in N$ of local $C^{\infty}$ real functions on $N$. The $\mathcal{O}_{N, a}$-module $(\mathcal{T} N)_{a}$ of germs at point $a$ of local sections of $T N$ is isomorphic to Der $\mathcal{O}_{N, a}$, the $\mathcal{O}_{N, a}$-module of derivations of $\mathcal{O}_{N, a}$. We denote by $L_{j}$ the $\mathcal{O}_{N, a}$-module of germs at point $a$ of local $C^{\infty}$-sections of $\left.R^{j}\right|_{N}$, considered as a vector bundle on $N$ by the map $\left.\pi\right|_{N}:\left.R^{k}\right|_{N} \rightarrow N$. Let be the $\mathcal{O}_{N, a}$-modules

$$
L=\lim \operatorname{proj} L_{j}
$$

and

$$
\mathcal{L}=\operatorname{Der} \mathcal{O}_{N, a} \oplus L
$$

The bilinear antisymmetric map

$$
\llbracket, \rrbracket_{k+l}:\left(\mathcal{T} \oplus \mathcal{R}^{k+l}\right) \times\left(\mathcal{T} \oplus \mathcal{R}^{k+l}\right) \rightarrow \mathcal{T} \oplus \mathcal{R}^{k+l-1}
$$

induces a well defined $\mathbb{R}$-bilinear antisymmetric map

$$
\llbracket, \rrbracket_{j}:\left(\operatorname{Der} \mathcal{O}_{N, a} \oplus L_{j}\right) \times\left(\operatorname{Der} \mathcal{O}_{N, a} \oplus L_{j}\right) \rightarrow \operatorname{Der} \mathcal{O}_{N, a} \oplus L_{j-1}
$$

As we saw in $(2.24)$, the projective limit of $\llbracket, \rrbracket_{j}$ induces a $\mathbb{R}$-Lie bracket

$$
\llbracket, \rrbracket_{\infty}: \mathcal{L} \times \mathcal{L} \rightarrow \mathcal{L}
$$

so that $\mathcal{L}$ is a $\mathbb{R}$-Lie algebra.

The structure of the Lie algebra $\mathcal{L}$ is the semi-direct product of the Lie algebras Der $\mathcal{O}_{N, a}$ and $L$, where the action of Der $\mathcal{O}_{N, a}$ on $L_{m}$ is given by the restriction to $N$ of $D_{H}: \mathcal{R}^{j} \rightarrow$ $\mathcal{H}^{*} \otimes \mathcal{R}^{j-1}$. If $v \in \operatorname{Der} \mathcal{O}_{N, a}$ and $\xi$ is a section of $\left.R^{j}\right|_{N}$ defined in a neighborhood of $a \in N$, we get (see Proposition 2.5)

$$
\llbracket v, \xi \rrbracket_{j}=i(v) D \xi .
$$

The map $\left(\rho_{1}\right)_{*}: \mathcal{L} \rightarrow$ Der $\mathcal{O}_{N, a}$ is the canonical projection given by the direct sum, and

$$
\llbracket \xi, f \eta \rrbracket_{\infty}=\left(\left(\rho_{1}\right)_{*} \xi\right)(f) \eta+f \llbracket \xi, \eta \rrbracket_{\infty},
$$

where $f \in \mathcal{O}_{N, a}, \xi, \eta \in \mathcal{L}$. The restriction of $\llbracket, \rrbracket_{\infty}$ to $L$ is $\mathcal{O}_{N, a}$-bilinear, so $L$ is a $\mathcal{O}_{N, a}$-Lie algebra. Each $L_{j}$ is a free $\mathcal{O}_{N, a}$-module finitely generated.

Definition 6.1. We call $\mathcal{L}$ the $\mathcal{O}_{N, a^{-}}$intransitive $\mathbb{R}$-Lie algebra associated with the formally integrable linear Lie equation $R^{k}$ at the point $a$ (and transversal $N$ ).

In particular, we denote by $\mathcal{D}(V)$ the $\mathcal{O}_{N, a}$-intransitive Lie algebra associated with the linear Lie equation $J^{0} V$, and call it the $\mathcal{O}_{N, a^{-}}$intransitive $\mathbb{R}$-Lie algebra associated with the involutive distribution $V$ at point $a \in M$. Clearly $\mathcal{L} \subset \mathcal{D}(V)$.

If $\mathcal{L}_{j}=\operatorname{Der} \mathcal{O}_{N, a} \oplus L_{j}$, then $\left(\mathcal{L}_{j}, \llbracket, \rrbracket_{j}\right)$ is called the truncated $\mathcal{O}_{N, a}$-intransitive $\mathbb{R}$-Lie algebra of order $j$ associated with $R^{k}$ at point $a$ (and transversal $N$ ). Then we can state the Theorem of [25] as:

Theorem 6.1. Let be $\mathcal{L}_{k+2} \subset \mathcal{D}_{k+2}(V)$ a truncated $\mathcal{O}_{N, a}$-intransitive $\mathbb{R}$-Lie algebra. Then there exists a vector sub-bundle $R^{\prime k+1} \subset J^{k+1} V$ such that: 
(i) $R^{\prime k}=\pi_{k} R^{\prime k+1}$ is a vector sub-bundle of $J^{k} V$;

(ii) $\llbracket \mathcal{T} \oplus \mathcal{R}^{\prime k+1}, \mathcal{T} \oplus \mathcal{R}^{\prime k+1} \rrbracket_{k+1} \subset \mathcal{T} \oplus \mathcal{R}^{\prime k}$;

(iii) the truncated $\mathcal{O}_{N, a}$-intransitive $\mathbb{R}$-Lie algebra associated with $R^{\prime k+1}$ is $\mathcal{L}_{k+1}=\pi_{k+1} \mathcal{L}_{k+2}$.

Furthermore, if $h_{k}=\left\{\xi \in \mathcal{L}_{k} \mid \pi_{k-1} \xi=0\right\}$ is 2-acyclic, then $R^{\prime k}$ is formally integrable.

The definitions of $\mathcal{L}$ and $\mathcal{D}(V)$ depend on the choice of the transversal $N$. Let's now introduce a notion of isomorphism inspired in Theorem 5.1 such that the intransitive Lie algebras obtained at point $a$ taking different transversal submanifolds are isomorphic. We maintain the notation of Section 5.

Suppose that $R^{k} \subset J^{k} V, R^{\prime k} \subset J^{k} V^{\prime}$ are formally integrable intransitive linear Lie equations, $N$ and $N^{\prime}$ submanifolds of $M$ and $M^{\prime}$ transversal to integral submanifolds of $V$ and $V^{\prime}$, respectively, and $\phi: N \rightarrow N^{\prime}$ a diffeomorphism, $a \in N, a^{\prime} \in N^{\prime}$, and $\phi(a)=a^{\prime}$. We denote also by $\phi$ the isomorphism of $\mathbb{R}$-algebras $\phi: \mathcal{O}_{N, a} \rightarrow \mathcal{O}_{N^{\prime}, a^{\prime}}$, defined by $\phi(f)=f \phi^{-1}$. Let be

$$
\Phi_{j+1}: N \rightarrow \mathcal{Q}_{\phi}^{j+1}
$$

$\alpha$-sections such that $\phi=\beta \Phi_{j+1}$ and $\pi_{j} \Phi_{j+1}=\Phi_{j}$ for $j \geq 0$. Put $\Phi=\lim \operatorname{proj} \Phi_{j}$. We get maps

$$
\left(\Phi_{j+1}\right)_{*}: \mathcal{D}_{j}(V) \rightarrow \mathcal{D}_{j}\left(V^{\prime}\right)
$$

and

$$
\Phi_{*}: \mathcal{D}(V) \rightarrow \mathcal{D}\left(V^{\prime}\right) .
$$

The map $\Phi_{*}$ is $\mathbb{R}$-linear, commutes with $\llbracket, \rrbracket_{\infty}$, and, if $f \in \mathcal{O}_{N, a}$ and $\xi \in \mathcal{D}(V)$, then

$$
\Phi_{*}(f \xi)=\phi(f) \Phi_{*}(\xi) .
$$

Definition 6.2. We say that $\Phi_{*}$ is an isomomorphism from intransitive Lie algebra $\mathcal{L} \subset \mathcal{D}(V)$ onto intransitive Lie algebra $\mathcal{L}^{\prime} \subset \mathcal{D}\left(V^{\prime}\right)$ if

$$
\Phi_{*} \mathcal{L}=\mathcal{L}^{\prime} .
$$

If $\Phi_{*}$ is an isomorphism, then $\mathcal{L}$ is said isomorphic to $\mathcal{L}^{\prime}$.

Proposition 6.1. Suppose that $R^{k} \subset J^{k} V$ is a formally integrable intransitive linear Lie equation, $a, b$ points of $M, N, N_{1}$ transversal to the orbits of $R^{k}$ through the points $a, b$, and $\mathcal{L}, \mathcal{L}_{1}$ the intransitive Lie algebras associated with $R^{k}$ at the points a, $b$ (and transversal $N, N_{1}$ ), respectively. Let be $\rho: M \rightarrow N$ the fibration (at least locally) defined by the leaves of $V$. If $\rho(a)=\rho(b)$, then the $\mathcal{O}_{N, a}$-intransitive Lie algebra $\mathcal{L}$ is isomorphic to the $\mathcal{O}_{N_{1}, b}$-intransitive Lie algebra $\mathcal{L}_{1}$.

Proof. If $x, y \in M, \rho(x)=\rho(y)$, there exists $X \in P^{m}$ with $\alpha(X)=x, \beta(X)=y$. Therefore, we can choose $\Phi_{j}: N \rightarrow P^{j}$ such that $\beta \Phi_{j}(N)=N_{1}$. Then

$$
\left(\Phi_{j}\right)_{*}\left(\left.T N \oplus R^{j-1}\right|_{N}\right)=\left.T N_{1} \oplus R^{j-1}\right|_{N_{1}} .
$$

It follows from the formal integrability of $P^{k}$ that we can choose the family $\left\{\Phi_{j}: j \geq 1\right\}$ such that $\Phi_{j+1}$ projects on $\Phi_{j}$, for $j \geq 1$. If $\Phi=\lim \operatorname{proj} \Phi_{j}$, then $\Phi_{*} \mathcal{L}=\mathcal{L}_{1}$.

With these definitions, we can state Corollary 5.2 as:

Theorem 6.2. Suppose that $R^{k} \subset J^{k} V$ is a linear Lie equation with symbol $g^{k} 2$-acyclic, and $R^{\prime k} \subset J^{k} V^{\prime}$ another linear Lie equation. Let be $a \in M, a^{\prime} \in M^{\prime}, N$ and $N^{\prime}$ transversal to the orbits of $R^{k}$ and $R^{\prime k}$ through the points a and $a^{\prime}, \mathcal{L}_{k}$ and $\mathcal{L}^{\prime}{ }_{k}$ the truncated intransitive Lie algebras associated with $R^{k}$ and $R^{\prime k}$, at points $a, a^{\prime}$ and tranversal $N$ and $N^{\prime}$, respectively. If there exists $\Phi: N \rightarrow \mathcal{Q}_{\phi}^{k+1}$ such that $\beta \Phi=\phi: N \rightarrow N^{\prime}, \phi(a)=a^{\prime}$, and $\Phi_{*} \mathcal{L}_{k}=\mathcal{L}^{\prime}{ }_{k}$, then $R^{k}$ at point a is formally isomorphic to $R^{\prime k}$ at point $a^{\prime}$. 


\section{Application}

As an application of this theory, we could utilize the definition of intransitive Lie algebras to obtain the intransitive linear Lie equations in the plane obtained by É. Cartan in [1]. We will be limited to classifying the first order intransitive linear Lie equations, with $\operatorname{dim} g^{1}=1$. This will include the example we presented in the introduction, which was not presented by Cartan in his table, suppressed by a nullity hypothesis.

Let be $V$ a 1-dimensional distribution on $\mathbb{R}^{2}$, which we can suppose is generated by the vector field $\frac{\partial}{\partial y}$. We will use the coordinate system $p_{j, l}, j, l \geq 0,0 \leq j+l \leq k$, in $J^{k} V$, defined by

$$
p_{j, l}\left(j_{(a, b)}^{k} \Theta\right)=\frac{\partial^{j+l} \theta}{\partial x^{j} \partial y^{l}}(a, b),
$$

where $\Theta(x, y)=\theta(x, y) \frac{\partial}{\partial y}$. Let's consider $(0,0)$ as point base and the transversal $N=\mathbb{R} \times\{0\}$. Then Der $\mathcal{O}_{N,(0,0)}$ is generated, as $\mathcal{O}_{N,(0,0)}$-module, by $\left.\frac{\partial}{\partial x}\right|_{N}$. Let be $g_{V}^{k}$ the symbol of $J^{k} V$. This symbol is generated by $f^{j, l} \otimes j^{0} \frac{\partial}{\partial y}$, where

$$
f^{j, l}=\frac{1}{j ! l !}(d x)^{j}(d y)^{l},
$$

with $j, l \geq 0$ and $j+l=k$ (cf. (2.34)). Then

$$
\llbracket \frac{\partial}{\partial x}, f^{j, l} \otimes j^{0} \frac{\partial}{\partial y} \rrbracket_{k}=-f^{j-1, l} \otimes j^{0} \frac{\partial}{\partial y} \in g_{V}^{k-1},
$$

and if $Y \in J^{k} V$ is such that $\pi_{0}(Y)=j^{0} \frac{\partial}{\partial y}$, then

$$
\llbracket Y, f^{j, l} \otimes j^{0} \frac{\partial}{\partial y} \rrbracket_{k}=f^{j, l-1} \otimes j^{0} \frac{\partial}{\partial y} \in g_{V}^{k-1} .
$$

Let be $R^{1} \subset J^{1} V$ a formally integrable linear Lie equation, with $R^{0}=J^{0} V$. Suppose $g^{1}$ is the symbol of $R^{1}$, with $\operatorname{dim} g^{1}=1$. Then $g^{1}$ is generated by an element $X_{1}=\left(A f^{1,0}+B f^{0,1}\right) \otimes j^{0} \frac{\partial}{\partial y}$, with $A(x, y)^{2}+B(x, y)^{2} \neq 0$.

Lemma 7.1. If $R^{2}$ is the prolongation of $R^{1}$, then the symbol $g^{2}$ of $R^{2}$ is generated by

$$
\left(A f^{1,0}+B f^{0,1}\right)^{2} \otimes j^{0} \frac{\partial}{\partial y} .
$$

Proof. Consider $Y_{0}, Y_{1} \in \mathcal{R}^{2}$ such that $\pi_{0} Y_{0}=j^{0} \frac{\partial}{\partial y}$ and $\pi_{1} Y_{1}=\left(A f^{1,0}+B f^{0,1}\right) \otimes j^{0} \frac{\partial}{\partial y}$ is a section of $g^{1}$. Let be $Y=\left(a_{20} f^{2,0}+a_{11} f^{1,1}+a_{02} f^{0,2}\right) \otimes j^{0} \frac{\partial}{\partial y}$ a section of $g^{2}$. Then

$$
\llbracket \frac{\partial}{\partial x}, Y \rrbracket_{2}=-\left(a_{20} f^{1,0}+a_{11} f^{0,1}\right) \in g^{1}
$$

so $\left(a_{20} f^{1,0}+a_{11} f^{0,1}\right)=\lambda\left(A f^{1,0}+B f^{0,1}\right)$, for a real function $\lambda$. In a similar way,

$$
\llbracket Y_{0}, Y \rrbracket_{2}=\left(a_{11} f^{1,0}+a_{02} f^{0,1}\right) \in g^{1},
$$

and $\left(a_{11} f^{1,0}+a_{02} f^{0,1}\right)=\mu\left(A f^{1,0}+B f^{0,1}\right)$, for some real function $\mu$. Therefore, we get $a_{20}=\lambda A$, $a_{11}=\lambda B=\mu A$ and $a_{02}=\mu B$. So, there exists $r$ such that $\lambda=r A$ and $\mu=r B$. From this, $a_{20}=r A^{2}, a_{11}=r A B$ and $a_{02}=r B^{2}$. Then

$$
Y=r\left(A f^{1,0}+B f^{0,1}\right)^{2} \otimes j^{0} \frac{\partial}{\partial y} .
$$


A similar argument shows that $g^{k}$ is one dimensional and is generated by $\left(A f^{1,0}+B f^{0,1}\right)^{k} \otimes$ $j^{0} \frac{\partial}{\partial y}$. Consider now the complex (4.3),

$$
0 \rightarrow g^{l+1} \stackrel{\delta}{\rightarrow} T^{*} \otimes g^{l} \stackrel{\delta}{\rightarrow} \wedge^{2} \mathcal{T}^{*} \otimes g^{l-1} \rightarrow 0
$$

for $l \geq 2$. As $\operatorname{dim} g^{k}=1$ and $\operatorname{dim} T=2$, this complex is clearly exact, so $g^{1}$ is 2 -acyclic.

Let's now verify conditions on the truncated intransitive Lie algebra $\mathcal{L}_{2}$. The $\mathcal{O}_{N,(0,0)}$-module $\mathcal{T} N_{(0,0)}$ is generated by

$$
Y_{-1}=\left.\frac{\partial}{\partial x}\right|_{N},
$$

and the generators of $L_{2}$ are $Y_{0}, Y_{1}$, defined in the proof of lemma and $Y_{2}=\frac{1}{2}\left(a f^{1,0}+b f^{0,1}\right)^{2} \otimes$ $j^{0} \frac{\partial}{\partial y}$, restricted to $N$, again denoted by the same letters. Here, $a(x)=A(x, 0)$ and $b(x)=$ $B(x, 0)$. We have

$$
\begin{aligned}
& \llbracket Y_{-1}, Y_{0} \rrbracket_{2}=b_{00} \pi_{1}\left(Y_{0}\right)+b_{01} \pi_{1}\left(Y_{1}\right), \quad \llbracket Y_{0}, Y_{1} \rrbracket_{2}=b \pi_{1}\left(Y_{0}\right)+a_{01} \pi_{1}\left(Y_{1}\right), \\
& \llbracket Y_{-1}, Y_{1} \rrbracket_{2}=-a \pi_{1}\left(Y_{0}\right)+b_{11} \pi_{1}\left(Y_{1}\right), \quad \llbracket Y_{0}, Y_{2} \rrbracket_{2}=b \pi_{1}\left(Y_{1}\right), \\
& \llbracket Y_{-1}, Y_{2} \rrbracket_{2}=-a \pi_{1}\left(Y_{1}\right), \quad \quad \llbracket Y_{1}, Y_{2} \rrbracket_{2}=0,
\end{aligned}
$$

for $a, b, b_{00}, b_{01}, b_{11}, a_{01}$ in $\mathcal{O}_{N,(0,0)}$. It follows from

$$
\llbracket Y_{-1}, \llbracket Y_{0}, Y_{1} \rrbracket_{2} \rrbracket_{1}=\llbracket \llbracket Y_{-1}, Y_{0} \rrbracket_{2}, \pi_{1}\left(Y_{1}\right) \rrbracket_{1}+\llbracket \pi_{1}\left(Y_{0}\right), \llbracket Y_{-1}, Y_{1} \rrbracket_{2} \rrbracket_{1}
$$

that $\left(\frac{\partial b}{\partial x}-a a_{01}-b b_{11}\right) \pi_{0}\left(Y_{0}\right)=0$, so

$$
\frac{\partial b}{\partial x}-a a_{01}-b b_{11}=0
$$

As $a(0)$ or $b(0)$ is not null, we can solve this equation for $a_{01}$ or $b_{11}$. Then we can find a truncated intransitive Lie algebra $\mathcal{L}_{2}$ that projects on $\mathcal{L}_{1}$. Now by Theorem 6.2 we classify the isomorphism class of $\mathcal{L}_{1}$. We must examine two cases:

Case 1. We suppose $b(0) \neq 0$. By dividing by $b$, we can suppose $b=1$. Then the algebra $\mathcal{L}_{1}$ is generated by $Y_{-1}, \pi_{0} Y_{0}, \pi_{1} Y_{1}$, and they satisfy, from above,

$$
\llbracket Y_{-1}, \pi_{1} Y_{0} \rrbracket_{1}=b_{00} \pi_{0}\left(Y_{0}\right), \quad \llbracket Y_{-1}, \pi_{1} Y_{1} \rrbracket_{1}=-a \pi_{0}\left(Y_{0}\right), \quad \llbracket \pi_{1} Y_{0}, \pi_{1} Y_{1} \rrbracket_{1}=\pi_{0}\left(Y_{0}\right) .
$$

Let be $\mathcal{L}_{1}^{\prime}$ a truncated intransitive Lie algebra generated by $X_{-1}, X_{0}$ and $X_{1}$, such that

$$
\llbracket X_{-1}, X_{0} \rrbracket_{1}=0, \quad \llbracket X_{-1}, X_{1} \rrbracket_{1}=0, \quad \llbracket X_{0}, X_{1} \rrbracket_{1}=\pi_{0}\left(X_{0}\right),
$$

and $f_{1}: \mathcal{L}_{1}^{\prime} \rightarrow \mathcal{L}_{1}$ defined by

$$
f_{1}\left(X_{-1}\right)=Y_{-1}+a \pi_{1}\left(Y_{0}\right)+b_{00} \pi_{1}\left(Y_{1}\right), \quad f_{1}\left(X_{0}\right)=\pi_{1}\left(Y_{0}\right), \quad f_{1}\left(X_{1}\right)=\pi_{1}\left(Y_{1}\right) .
$$

Let's verify that $f_{1}$ is an isomorphism of truncated intransitive Lie algebras of order 1 . In fact,

$$
\begin{aligned}
& \llbracket f_{1}\left(X_{-1}\right), f_{1}\left(X_{0}\right) \rrbracket_{1}=\llbracket Y_{-1}+a \pi_{1}\left(Y_{0}\right)+b_{00} \pi_{1}\left(Y_{1}\right), \pi_{1}\left(Y_{0}\right) \rrbracket_{1}=0=f_{0}\left(\llbracket X_{-1}, X_{0} \rrbracket_{1}\right), \\
& \llbracket f_{1}\left(X_{-1}\right), f_{1}\left(X_{1}\right) \rrbracket_{1}=\llbracket Y_{-1}+a \pi_{1}\left(Y_{0}\right)+b_{00} \pi_{1}\left(Y_{1}\right), \pi_{1}\left(Y_{1}\right) \rrbracket_{1}=0=f_{0}\left(\llbracket X_{-1}, X_{1} \rrbracket_{1}\right),
\end{aligned}
$$

and

$$
\llbracket f_{1}\left(X_{0}\right), f_{1}\left(X_{1}\right) \rrbracket_{1}=\llbracket \pi_{1}\left(Y_{0}\right), \pi_{1}\left(Y_{1}\right) \rrbracket_{1}=\pi_{0}\left(Y_{0}\right)=f_{0}\left(\pi_{0}\left(X_{0}\right)\right)=f_{0}\left(\llbracket X_{0}, X_{1} \rrbracket_{1}\right) .
$$


The truncated Lie algebra $\mathcal{L}_{1}^{\prime}$ generated as $\mathcal{O}_{N,(0,0)}$-module by $X_{-1}, X_{0}, X_{1}$ can be represented by

$$
X_{-1}=\left.\frac{\partial}{\partial x}\right|_{N}, \quad X_{0}=j^{1} \frac{\partial}{\partial y}, \quad X_{1}=f^{0,1} \otimes j^{0} \frac{\partial}{\partial y} .
$$

The linear Lie equation associated with $\mathcal{L}_{1}^{\prime}$ is

$$
R^{\prime 1}=\left\{\left(p_{0,0}, p_{1,0}, p_{0,1}\right) \in J^{1} V: p_{1,0}=0\right\},
$$

so $R^{1}$ is formally isomorphic to $R^{\prime 1}$. The infinitesimal pseudogroup of solutions of $R^{\prime 1}$ is

$$
\left\{\Theta(x, y)=\theta(y) \frac{\partial}{\partial y}\right\}
$$

Case 2. We suppose $b(0)=0$. In this case, $a(0) \neq 0$, and, dividing it by $a$, we can suppose $a=1$. Then the truncated intransitive Lie algebra $\mathcal{L}_{1}$ is generated by $Y_{-1}, \pi_{0} Y_{0}, \pi_{1} Y_{1}$, and they satisfy, from above,

$$
\llbracket Y_{-1}, \pi_{1} Y_{0} \rrbracket_{1}=b_{00} \pi_{0}\left(Y_{0}\right), \quad \llbracket Y_{-1}, \pi_{1} Y_{1} \rrbracket_{1}=-\pi_{0}\left(Y_{0}\right), \quad \llbracket \pi_{1} Y_{0}, \pi_{1} Y_{1} \rrbracket_{1}=b \pi_{0}\left(Y_{0}\right) .
$$

Replacing $\pi_{1} Y_{0}$ by $\pi_{1} Y_{0}+b_{00} \pi_{1} Y_{1}$, we obtain $\llbracket Y_{-1}, \pi_{1} Y_{0}+b_{00} \pi_{1} Y_{1} \rrbracket_{1}=0$, and the other products remain unchanged. Without loss of generality, we can suppose $b_{00}=0$. Let be $\mathcal{L}_{1}^{\prime}$ a truncated intransitive Lie algebra generated by $X_{-1}, X_{0}$ and $X_{1}$, such that

$$
\llbracket X_{-1}, X_{0} \rrbracket_{1}=0, \quad \llbracket X_{-1}, X_{1} \rrbracket_{1}=-\pi_{0}\left(X_{0}\right), \quad \llbracket X_{0}, X_{1} \rrbracket_{1}=\beta \pi_{0}\left(X_{0}\right),
$$

and $f_{1}: \mathcal{L}_{1}^{\prime} \rightarrow \mathcal{L}_{1}$ defined by

$$
f_{1}\left(X_{-1}\right)=c^{-1} Y_{-1}, \quad f_{1}\left(X_{0}\right)=\pi_{1}\left(Y_{0}\right), \quad f_{1}\left(X_{1}\right)=c \pi_{1}\left(Y_{1}\right),
$$

with $c(0) \neq 0$. Then

$$
\begin{aligned}
& \llbracket f_{1}\left(X_{-1}\right), f_{1}\left(X_{0}\right) \rrbracket_{1}=\llbracket c^{-1} Y_{-1}, \pi_{1}\left(Y_{0}\right) \rrbracket_{1}=0=f_{0}\left(\llbracket X_{-1}, X_{0} \rrbracket_{1}\right), \\
& \llbracket f_{1}\left(X_{-1}\right), f_{1}\left(X_{1}\right) \rrbracket_{1}=\llbracket c^{-1} Y_{-1}, c \pi_{1}\left(Y_{1}\right) \rrbracket_{1}=-\pi_{0}\left(Y_{0}\right)=f_{0}\left(\llbracket X_{-1}, X_{1} \rrbracket_{1}\right),
\end{aligned}
$$

and

$$
\llbracket f_{1}\left(X_{0}\right), f_{1}\left(X_{1}\right) \rrbracket_{1}=\llbracket \pi_{1}\left(Y_{0}\right), c \pi_{1}\left(Y_{1}\right) \rrbracket_{1}=c b \pi_{0}\left(Y_{0}\right)=f_{0}\left(\beta \pi_{0}\left(X_{0}\right)\right)=f_{0}\left(\llbracket X_{0}, X_{1} \rrbracket_{1}\right),
$$

if $c b=\beta$.

Then $f_{1}$ is an isomorphism of truncated intransitive Lie algebras of order 1 if and only if there exists $c \in \mathcal{O}_{N,(0,0)}$ with $c(0) \neq 0$ such that $c b=\beta$. The class of truncated intransitive Lie algebras is the class of equivalence of $b, b(0)=0$, and $b \equiv \beta$ if and only if there exists $c, c(0) \neq 0$, with $b c=\beta$.

The truncated Lie algebra $\mathcal{L}_{1}^{\prime}$ generated as $\mathcal{O}_{\mathbb{R}}$-module by $X_{-1}, X_{0}, X_{1}$ can be represented by

$$
X_{-1}=\frac{\partial}{\partial x}, \quad X_{0}=j^{1} \frac{\partial}{\partial y}, \quad X_{1}=\left(f^{1,0}+\beta f^{0,1}\right) \otimes j^{0} \frac{\partial}{\partial y},
$$

and the linear Lie equation associated with $\mathcal{L}_{1}^{\prime}$ is

$$
R^{\prime 1}=\left\{\left(p_{0,0}, p_{1,0}, p_{0,1}\right) \in J^{1} V: p_{0,1}=\beta p_{1,0}\right\},
$$


so $R^{1}$ is formally isomorphic to $R^{\prime 1}$. The infinitesimal pseudogroup of solutions of $R^{\prime 1}$ is

$$
\left\{\Theta(x, y)=\theta(x, y) \frac{\partial}{\partial y}: \frac{\partial \theta}{\partial y}=\beta \frac{\partial \theta}{\partial x}\right\}
$$

If $\beta(x)=x$, we obtain

$$
\left\{\Theta(x, y)=\theta\left(x e^{y}\right) \frac{\partial}{\partial y}\right\}
$$

if $\beta(x)=x^{k}, k \geq 2$, we obtain

$$
\left\{\Theta(x, y)=\theta\left(\frac{x^{k-1}}{(k-1) y x^{k-1}-1}\right) \frac{\partial}{\partial y}\right\}
$$

if $\beta(x)=0$, we obtain

$$
\left\{\Theta(x, y)=\theta(x) \frac{\partial}{\partial y}\right\}
$$

In the classification of [1], case 2 is represented only by $\beta=0$.

\section{Conclusion}

The results of this paper show that the intransitive Lie algebra here introduced to represent a linear Lie equation at a point is sufficient to guarantee the existence and formal isomorphism of intransitive linear Lie equations. This brings a new way to pursue the study of intransitive Lie groups and the applications envisaged by Sophus Lie on the integrability of partial differential equations with a pseudogroup of invariants. It is clear that several problems can still exist, as the relationship between subalgebras of transitive algebras and intransitive algebras, and the notion of equivalence of intransitive algebras. A very interesting problem is the classification of simple intransitive Lie groups, since Cartan, in his list, excluded some classes of simple intransitive Lie group, as the example presented above.

\section{Acknowledgments}

I would like to thank the referees for the several suggestions to improve this paper.

\section{References}

[1] Cartan É., Sur le structure des groupes infinis de transformations, Ann. Sci. École Norm. Sup. (3) 21 (1904), 153-206.

Cartan É., Sur la structure des groupes infinis de transformation (suite), Ann. Sci. École Norm. Sup. (3) 22 (1905), 219-308.

[2] Conn J.F., A new class of counterexamples to the integrability problem, Proc. Nat. Acad. Sci. USA 74 (1977), 2655-2658.

[3] Frölicher A., Nijenhuis A., Theory of vector valued differential forms. I. Derivations in the graded ring of differential forms, Nederl. Akad. Wet. Proc. Ser. A 59 (1956), 338-359.

[4] Goldschmidt H., Existence theorems for analytic linear partial differential equations, Ann. of Math. (2) 86 (1967), 246-270.

[5] Goldschmidt H., Integrability criteria for systems of non-linear partial differential equations, J. Differential Geom. 1 (1967), 269-307. 
[6] Goldschmidt H., Sur la structure des équations de Lie. I. Le troisième théorème fondamental, J. Differential Geom. 6 (1972), 357-373.

[7] Goldschmidt H., Sur la structure des équations de Lie. II. Équations formellement transitives, J. Differential Geom. 7 (1972). 67-95.

[8] Goldschmidt H., Sur la structure des équations de Lie. III. La cohomologie de Spencer, J. Differential Geom. 11 (1976). 167-223.

[9] Goldschmidt H., Spencer D., On the non-linear cohomology of Lie equations. I, Acta Math. 136 (1976), 103-170.

Goldschmidt H., Spencer D., On the non-linear cohomology of Lie equations. II, Acta Math. 136 (1976), $171-239$.

[10] Guillemin V.W., Sternberg S., An algebraic model of transitive differential geometry, Bull. Amer. Math. Soc. 70 (1964), 16-47.

[11] Guillemin V.W., Sternberg S., The Lewy counterexample and the local equivalence problem for $G$-structures, J. Differential Geom. 1 (1967), 127-131.

[12] Kiso K., Local properties of intransitive infinite Lie algebra sheaves, Japan. J. Math. (N.S.) 5 (1979), $101-155$.

[13] Kiso K., Infinitesimal automorphisms of $G$-structures and certain intransitive infinite Lie algebra sheaves, Hokkaido Math. J. 11 (1982), 301-327.

[14] Kumpera A., Spencer D.C., Lie equations, Vol. I, General theory, Annals of Mathematics Studies, Vol. 73, Princeton University Press, Princeton, N.J.; University of Tokyo Press, Tokyo, 1972.

[15] Kamber F.W., Tondeur P., Foliated bundles and characteristic classes, Lecture Notes in Mathematics, Vol. 493, Springer-Verlag, Berlin - New York, 1975.

[16] Malgrange B., Equations de Lie. I, J. Differential Geom. 6 (1972), 503-522.

[17] Malgrange B., Equations de Lie. II, J. Differential Geom. 7 (1972), 117-141.

[18] Morimoto T., On the intransitive Lie algebras whose transitive parts are infinite and primitive, J. Math. Soc. of Japan 29 (1977), 35-65.

[19] Ngô Van Quê V.V.T., Définition des pseudogroupes infinitesimaux de Lie intransitifs. Théorème fondamental de réalization en dimension deux, Nagoya Math. J. 86 (1982), 211-228.

[20] Petitjean A., Rodrigues A.M., Correspondance entre algébres de Lie abstraites et pseudo-groupes de Lie transitifs, Ann. of Math. (2) 101 (1975), 268-279.

[21] Ruiz C., Prolongement formel des systèmes différentiels extérieur d'ordre supérieur, C. R. Acad. Sci. Paris Sér. A-B 285 (1977), A1077-A1080.

[22] Ruiz C., Complexe de Koszul du symbole d'un système différentiel extérieur, C. R. Acad. Sci. Paris Sér. A-B 286 (1978), A55-A58.

[23] Ruiz C., Propriétès de dualité du prolongement formel des systèmes différentiels extérieurs, $C$. R. Acad. Sci. Paris Sér. A-B 286 (1978), A99-A101.

[24] Singer I.M., Sternberg S., The infinite groups of Lie and Cartan. I. The transitive groups, J. Analyse Math. 15 (1965), 1-114.

[25] Veloso J.M.M., Lie's third theorem for intransitive Lie equations, J. Differential Geom. 32 (1990), $185-198$.

[26] Veloso J.M., Prolongation projection commutativity theorem, in CR-Geometry and Overdetermined Systems (Osaka, 1994), Adv. Stud. Pure Math., Vol. 25, Math. Soc. Japan, Tokyo, 1997, 386-405.

[27] Veloso J.M.M., New classes of intransitive simple Lie pseudogroups, Bull. Soc. Sci. Lett. Lodz 36 (1986), no. 19,7 pages. 